\title{
Towards Feynman rules for conformal blocks
}

\author{
Sarah Hoback ${ }^{a}$ and Sarthak Parikh ${ }^{b}$ \\ ${ }^{a}$ Department of Physics and Astronomy, Pomona College, \\ $333 \mathrm{~N}$ College Way, Claremont, CA 91711, U.S.A. \\ ${ }^{b}$ Division of Physics, Mathematics and Astronomy, California Institute of Technology, \\ 1200 E California Blvd, Pasadena, CA 91125, U.S.A. \\ E-mail: sarahhoback98@gmail.com, sparikh@caltech.edu
}

ABSTRACT: We conjecture a simple set of "Feynman rules" for constructing $n$-point global conformal blocks in any channel in $d$ spacetime dimensions, for external and exchanged scalar operators for arbitrary $n$ and $d$. The vertex factors are given in terms of Lauricella hypergeometric functions of one, two or three variables, and the Feynman rules furnish an explicit power-series expansion in powers of cross-ratios. These rules are conjectured based on previously known results in the literature, which include four-, five- and six-point examples as well as the $n$-point comb channel blocks. We prove these rules for all previously known cases, as well as two new ones: the seven-point block in a new topology, and all even-point blocks in the "OPE channel." The proof relies on holographic methods, notably the Feynman rules for Mellin amplitudes of tree-level AdS diagrams in a scalar effective field theory, and is easily applicable to any particular choice of a conformal block beyond those considered in this paper.

KEYwords: Conformal Field Theory, AdS-CFT Correspondence, Conformal and W Symmetry

ArXiv EPrint: 2006.14736 


\section{Contents}

1 Introduction 1

2 Feynman rules for conformal blocks 4

2.1 Mellin and post-Mellin parameters $\quad 7$

3 Examples $\quad 10$

$\begin{array}{lll}3.1 & \text { Seven-point mixed channel block } & 10\end{array}$

$\begin{array}{ll}3.2 n \text {-point comb channel block } & 15\end{array}$

$\begin{array}{lll}3.3 & n \text {-point OPE channel block } & 19\end{array}$

4 From Mellin amplitudes to conformal blocks $\quad 24$

$\begin{array}{lll}4.1 & \text { Seven-point mixed channel } & 28\end{array}$

4.2 Comb channel 33

$\begin{array}{lll}4.3 & \text { OPE channel } & 39\end{array}$

$\begin{array}{lll}5 & \text { Discussion } & 48\end{array}$

$\begin{array}{ll}\text { A Lauricella functions } & 49\end{array}$

B Technical details $\quad \mathbf{5 0}$

B.1 Proof of (3.46) 50

B.2 Proof of (4.38) 51

B.3 OPE channel calculations $\quad 52$

B.3.1 Base case $\quad 52$

$\begin{array}{lll}\text { B.3.2 Integrals over a green-colored chain } & 59\end{array}$

B.3.3 Integrals over a magenta-colored chain 62

\section{Introduction}

Conformal blocks are theory-independent building blocks of conformal field theories (CFTs) which capture contributions to conformal correlators from entire conformal families of representations appearing in the intermediate channels of correlation functions. Via the AdS/CFT correspondence, they play an important role in the gravitational context as well; for example they provide a basis for writing down any bulk Witten diagram.

Conformal blocks also play a crucial, central role in the revived conformal bootstrap program [1-3] (see also the recent review [4] and references therein), which has led to significant advances in understanding properties of $d$-dimensional CFTs as well as holography. This has resulted in considerable interest in and a spate of new results for conformal blocks. 
However, until recently, much of the focus has been restricted to four-point conformal blocks [5-10]. A variety of techniques are now available for obtaining four-point global conformal blocks for arbitrary external and exchanged representations in the intermediate channels in various forms such as closed-form, integral or series representations. A partial list of methods includes various recursive techniques, shadow formalism, use of differential operators, dimensional reduction, integrability methods, and holographic geodesic diagram techniques $[8,10-52]$.

The focus on four-point blocks is due in part to the fact that conformal bootstrap is typically implemented at the level of four-point correlators. This is expected to be sufficient for constraining the full CFT data as long as one includes crossing-symmetry constraints from all possible four-point correlators, including those with arbitrary representations at external legs. This can be non-trivial and computationally very costly to implement. An alternative to this approach may be an $n$-point bootstrap program restricted simply to external scalars [53]. Implementing this approach would necessarily require the knowledge of higher-point scalar conformal blocks in arbitrary channels.

Recently, bulk unitarity methods [54] have also clarified the role of higher-point treelevel AdS diagrams in four-point results beyond the planar limit, i. e. in understanding the properties of higher-loop corrections. Higher-point tree-level AdS diagrams in turn are easily expressible via a conformal block decomposition or via a spectral representation in terms of direct channel conformal blocks and leading OPE coefficients. Thus the knowledge of higher-point conformal blocks in arbitrary channels would be particularly useful in probing holography at higher-loops.

However, obtaining explicit representations for conformal blocks is a notoriously hard problem, even though in principle the blocks are fixed entirely by conformal symmetry. The challenges are particularly pronounced in the case of higher-point blocks in $d$ spacetime dimensions, where until recently hardly any results were available. The $d$-dimensional five-point block was obtained using the shadow formalism in ref. [53] (see also refs. [55, 56]). A holographic representation for the five-point block was worked out in ref. [55], and subsequently extended to the six-point block in the so-called "OPE channel" [57], as well as to higher-point blocks in the comb channel [58]. Ref. [58] also worked out an explicit power-series expansion for the $n$-point comb channel blocks. CFT embedding space methods [28, 30] have also been fruitful in yielding higher-point blocks [59]; notably providing a series expansion for the $n$-point comb channel block [60], and the six-point block in the OPE channel, referred to as the "snowflake channel" [61]. ${ }^{1}$

While the recent burst of activity and progress in studying higher-point functions and conformal blocks is encouraging, the situation is far from settled. A particularly troubling aspect of going to higher-point blocks is that the number of possible inequivalent channels grows very rapidly with $n$, thus it seems highly inefficient and impractical to work out the associated conformal blocks on a case by case basis. What would be desirable is a set of

\footnotetext{
${ }^{1}$ See also ref. [62] for an application to two-dimensional six-point global blocks for stress tensor exchanges, and ref. [63] for obtaining representations of (higher-point) diagrams in two and four spacetime dimensions in terms of solutions to Lauricella systems for conformal groups $\operatorname{SL}(2, \mathbb{C})$ and $\operatorname{SL}(2, \mathbb{H})$. Recent progress in higher-point diagrams has also come via momentum space techniques [64-67].
} 
Feynman-like rules which could be determined once and for all, that enable writing down any conformal block in any topology without having to do any computations.

Motivated by these considerations, in this paper we will present a simple, conjectural prescription for writing down an arbitrary $d$-dimensional $n$-point scalar conformal block with scalar exchanges in any given channel. Even though the blocks themselves are non-perturbative objects, we call them "Feynman rules" because they are reminiscent of Feynman rules for Mellin amplitudes [68-70]. This conjecture was motivated by carefully studying the power-series expansions of all known examples of scalar conformal blocks in the literature, particularly as presented in refs. [53, 55, 57, 58, 60, 61].

As a highly non-trivial check of these rules, we compare the predicted blocks belonging to an infinite family of blocks previously unknown in the literature against a first-principles derivation and find exact agreement. These are the $n$-point conformal blocks in the so-called "OPE channel" for arbitrary even $n$. We also test the rules in the case of a seven-point block in a topology different from the comb channel, which we simply refer to as the "mixed channel," and find perfect agreement.

The key idea which enables us to compute these new families of blocks from first principles was previously utilized in refs. $[55,57,58]$ to obtain the holographic duals of higher-point blocks. To obtain a particular scalar conformal block, we start with a treelevel Witten diagram in a cubic $\phi^{3}$ effective field theory whose direct channel conformal block decomposition admits the desired block as its single-trace contribution. We call such a Witten diagram the "canonical Witten diagram" for the block, and there is a unique choice for each conformal block. Then the single-trace contribution to the canonical Witten diagram is given by the desired block times a set of known mean field theory OPE coefficients. Thus the key step is to obtain the single-trace projection of the Witten diagram, as this will immediately yield the conformal block.

Here, we appeal to Mellin space technology [71-73], which serves a two-fold purpose. Firstly, in a large $N$ bulk theory, Mellin amplitudes are meromorphic functions with poles corresponding precisely to the exchange of single-trace operators; this provides a convenient route to single-trace projections. Secondly, Mellin amplitudes for all tree-level scalar Witten diagrams in scalar effective field theories are known (thanks to the Mellin space Feynman rules [68-70]); this enables us to obtain an explicit single-trace projection of any $n$-point canonical Witten diagram. This method of projecting out the multi-trace exchanges to obtain the conformal block is quite general, efficient and constructive, so it can be used to work out any particular conformal block. The main, and often only, computationally challenging step of this procedure will be the actual evaluation of all residual Mellin integrals, which is required to obtain an explicit power-series expansion for the block. However, in all examples we attempted we were able to systematically work out all such integrals merely by repeated, and often inductive, applications of the first Barnes lemma [74].

The outline for the rest of the paper is as follows: in section 2 we propose the Feynman rules for conformal blocks, and in section 3 we illustrate how to apply them to obtain a seven-point block in the "mixed channel," the $n$-point comb channel block, and the $n$-point OPE channel block. In section 4 we revisit all examples from section 3 and using the Mellin-space single-trace projection technique, we prove the Feynman rules in each case. 


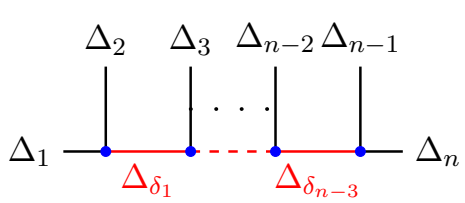

(a)

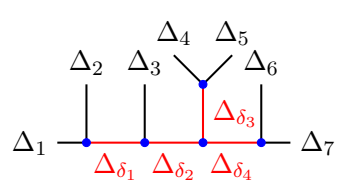

(b)

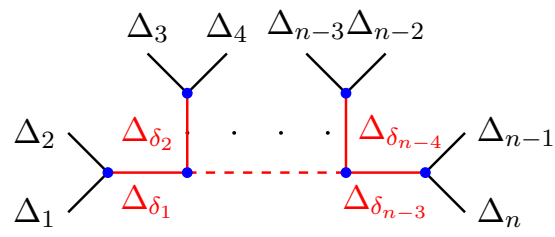

(c)

Figure 1. Graphical representation of conformal blocks: any $n$-point block admits a unique representation as an unrooted binary tree with $n$ leaves, and consequently $n-3$ internal edges (colored red to guide the eye) and $n-2$ internal nodes/vertices (marked in blue). All edges are labeled with conformal dimensions; the labels on external edges (edges attached to the leaves of the unrooted tree) are shown at the leaves for better presentation. (a): the graph shows an $n$-point "comb channel" conformal block (for $n \geq 4$ ) for external scalar operators $\mathcal{O}_{1}\left(x_{1}\right), \ldots, \mathcal{O}_{n}\left(x_{n}\right)$ with conformal dimensions $\Delta_{1}, \ldots, \Delta_{n}$ and insertion coordinates $x_{1}, \ldots, x_{n}$ respectively, and exchanged scalar operators $\mathcal{O}_{\delta_{1}}, \ldots, \mathcal{O}_{\delta_{n-3}}$ along the internal edges with conformal dimensions $\Delta_{\delta_{1}}, \ldots, \Delta_{\delta_{n-3}}$, respectively. (c): the graph shows an $n$-point block in the "OPE channel," for even $n \geq 6$. One can obtain the $n$-point OPE channel topology by starting with an $\frac{n}{2}$-point comb channel block and attaching two external edges at every leaf. (b): the graph shows a 7-point example in a "mixed channel" which is neither the comb nor the OPE channel.

We end with some discussion and future directions in section 5. Various technical details and computations are provided in the appendices.

When this work was largely complete, we learned of parallel, independent work to appear by Fortin, Ma and Skiba [75], which has partial overlap with some results of this paper.

\section{Feynman rules for conformal blocks}

Given any $n$-point conformal block, let the dimensions and insertion coordinates of the external operators be respectively, $\Delta_{i}$ and $x_{i}$ for $i=1, \ldots, n$. Let the dimensions of the exchanged operators be enumerated $\Delta_{\delta_{i}}$ for $i=1, \ldots, n-3$. See figure 1 for some examples of graphical representation of blocks in different channels as unrooted binary trees with $n$ leaves (and correspondingly $n-2$ internal vertices and $n-3$ internal edges), which will play a central role in the Feynman rules. Different inequivalent channels/topologies correspond to different OPE structures which can contribute to a conformal correlation function.

The Feynman rules presented here give an expression for the desired conformal block in the desired channel as an $n(n-3) / 2$-fold power series in powers of $n(n-3) / 2$ independent cross-ratios built out of operator insertion positions $x_{i}{ }^{2}$ The set of cross-ratios will be treated as input data fed into the rules to obtain the conformal block. We will assume the independent cross-ratios are enumerated by $u_{i}$ for $i=1, \ldots, n-3$ and $v_{j}$ for $j=$ $1, \ldots,\left(\begin{array}{c}n-2 \\ 2\end{array}\right)$, such that under any OPE limit, one or more of only $u_{i}$-type cross-ratios tend to zero. On the other hand, under any OPE limit a subset of $v_{j}$-type cross-ratios tend to unity, while a subset simplifies to give duplicated $v_{j}$ cross-ratios. Upon repeatedly taking

\footnotetext{
${ }^{2}$ We assume sufficiently high $d$. Otherwise some of the cross-ratios will be dependent, but the prescription still works. In this case, however, a more efficient power series with fewer overall sums also exists.
} 
sufficiently many OPE limits, we can arrange for all $u_{i} \approx 0$ and all $v_{j} \approx 1$. In this limit the leading contribution to the conformal block is given by

$$
\left.W_{n}\left(x_{i}\right)\right|_{\substack{u_{i} \approx 0 \\ v_{j} \approx 1}} \propto \prod_{i=1}^{n-3} u_{i}^{\Delta_{\delta_{i}} / 2}+\cdots
$$

There is a choice in picking $n(n-3) / 2$ independent cross-ratios subject to the constraint (2.1). The Feynman rules described here, which treat the cross-ratios as input data, work for any such choice. From here on, we fix a choice.

Away from the limit (2.1), the conformal block admits an expansion of the form

$$
W_{n}\left(x_{i}\right)=W_{n}^{0}\left(x_{i}\right)\left(\prod_{i=1}^{n-3} u_{i}^{\Delta_{\delta_{i}} / 2}\right) g(u, 1-v)
$$

where $W_{n}^{0}\left(x_{i}\right)$, which will be referred to as the "leg factor," depends only on position coordinates $x_{i}$ and external dimensions $\Delta_{i}$ and captures the overall scaling behavior of the conformal block under conformal transformations. The function $g(u, 1-v)$ is expressed as a power series in $u_{i}$ and $\left(1-v_{j}\right)$ for all $i, j$, with the leading behaviour $g(u, 1-v)=$ $1+O\left(u_{i}, 1-v_{j}\right)$. This function sums all descendant contributions to the conformal block. The Feynman rules provide a prescription for writing down this function in terms of "edge factors" $E_{i}$ and "vertex factors" $V_{i}$ associated respectively with each internal edge and internal vertex of the unique unrooted binary tree representation of the desired conformal block (see e.g. figure 1):

$$
g(u, 1-v)=\sum_{k_{i}, j_{r s}=0}^{\infty}\left[\left(\prod_{i=1}^{n-3} \frac{u_{i}^{k_{i}}}{k_{i} !}\right)\left(\prod_{(r s)}^{\left(\begin{array}{c}
n-2 \\
2
\end{array}\right)} \frac{\left(1-v_{r s}\right)^{j_{r s}}}{j_{r s} !}\right)\left(\prod_{i=1}^{n-3} E_{i}\right)\left(\prod_{i=1}^{n-2} V_{i}\right)\right]
$$

The position-independent edge and vertex factors depend solely on the external and exchanged conformal dimensions, as well as the non-negative integral parameters being summed over, $k_{i}$ and $j_{r s}$, where $i=1, \ldots, n-3$ and the $(r s)$ index takes $\left(\begin{array}{c}n-2 \\ 2\end{array}\right)$ values. (For convenience we have also re-enumerated the $v_{j}$ cross-ratios as $v_{r s}$; the precise mapping will be explained shortly.) They are determined as follows:

- Label each internal edge with an index $i$ running from 1 to $n-3$, such that the conformal dimension of the exchanged operator running along the edge is twice the exponent of the cross-ratio $u_{i}$ appearing in (2.1). Associate to each such edge an integral parameter $k_{i}$ and a factor of

$$
E_{i}:=\frac{\left(\Delta_{\delta_{i}}-h+1\right)_{k_{i}}}{\left(\Delta_{\delta_{i}}\right)_{2 k_{i}+\ell_{\delta_{i}}}}
$$

where $\Delta_{\delta_{i}}$ is the conformal dimension of the exchanged operator running along the edge, and $\ell_{\delta_{i}}$ is an integral parameter associated with the conformal dimension $\Delta_{\delta_{i}}$ to be determined later. Here $(a)_{b} \equiv \Gamma(a+b) / \Gamma(a)$ is the Pochhammer symbol, and we have defined

$$
h:=d / 2
$$


We refer to the parameters $k_{i}$ as "single-trace parameters," and the parameters $\ell_{\delta_{i}}$ as "post-Mellin parameters." The single-trace parameter $k_{i}$ also appears in the series expansion (2.3) as the exponent of the cross-ratio $u_{i}$. The post-Mellin parameters are specified entirely in terms of specific positive linear combinations of the parameters $j_{r s}$ appearing in (2.3), which we call "Mellin parameters." The precise relation between the two will be discussed in section 2.1. In that section, we will also present an alternate prescription for assigning the appropriate single-trace parameter to each internal edge.

- Label each internal (i.e. cubic) vertex with an index $i$ running from 1 to $n-2 \cdot{ }^{3}$ Let the number of incident internal edges on it be denoted $M$. It is clear that $M$ can only be 1,2 , or 3 . Let the conformal dimensions attached to the edges be $\Delta_{a}, \Delta_{b}$, and $\Delta_{c}$. Consider first a vertex with $M=3$ (i.e. with all incident edges internal). To this vertex, assign a factor of

$$
\begin{aligned}
V_{i}:= & \left(\Delta_{a b, c}\right)_{k_{a b, c}+\frac{1}{2} \ell_{a b, c}}\left(\Delta_{a c, b}\right)_{k_{a c, b}+\frac{1}{2} \ell_{a c, b}}\left(\Delta_{b c, a}\right)_{k_{b c, a}+\frac{1}{2} \ell_{b c, a}} \\
& \times F_{A}^{(3)}\left[\Delta_{a b c,}-h ;\left\{-k_{a},-k_{b},-k_{c}\right\} ;\left\{\Delta_{a}-h+1, \Delta_{b}-h+1, \Delta_{c}-h+1\right\} ; 1,1,1\right]
\end{aligned}
$$

where $F_{A}^{(3)}$ is the Lauricella function of three variables, defined in (A.1). Here $k_{a}, k_{b}$, and $k_{c}$ are the respective single-trace parameters associated with each internal edge above, and $\ell_{a}, \ell_{b}$, and $\ell_{c}$ are the post-Mellin parameters associated with $\Delta_{a}, \Delta_{b}$ and $\Delta_{c}$, respectively. Here and below, we are using the shorthand,

$$
\Delta_{i_{1} \ldots i_{m}, i_{m+1} \ldots i_{n}}:=\frac{1}{2}\left(\Delta_{i_{1}}+\cdots+\Delta_{i_{m}}-\Delta_{i_{m+1}}-\cdots-\Delta_{i_{n}}\right)
$$

for conformal dimensions $\Delta_{i}$, whereas for single-trace parameters and post-Mellin parameters we are using

$$
\begin{aligned}
& k_{i_{1} \ldots i_{m}, i_{m+1} \ldots i_{n}}:=k_{i_{1}}+\cdots+k_{i_{m}}-k_{i_{m+1}}-\cdots-k_{i_{n}} \\
& \ell_{i_{1} \ldots i_{m}, i_{m+1} \ldots i_{n}}:=\ell_{i_{1}}+\cdots+\ell_{i_{m}}-\ell_{i_{m+1}}-\cdots-\ell_{i_{n}} .
\end{aligned}
$$

For a vertex with $M=2$ (respectively, $M=1$ ), one (respectively, two) of the incident edges is an external edge. So far, external edges have not been assigned a single-trace parameter. It is convenient to view an external edge as an edge with its single-trace parameter set to zero. Then the vertex factor continues to be given by (2.6), but with the associated single-trace parameter(s) set to zero.

It is worth noting that the Lauricella function $F_{A}^{(3)}$ in (2.6) with say, $k_{a}=0$ reduces to the Lauricella function of two variables,

$$
F_{A}^{(2)}\left[\Delta_{a b c},-h ;\left\{-k_{b},-k_{c}\right\} ;\left\{\Delta_{b}-h+1, \Delta_{c}-h+1\right\} ; 1,1\right] .
$$

Likewise if two of the attached edges are external, with say, $k_{a}=k_{b}=0$, then the Lauricella function reduces further to the Lauricella function of one variable,

$$
F_{A}^{(1)}\left[\Delta_{a b c,}-h ;\left\{-k_{c}\right\} ;\left\{\Delta_{c}-h+1\right\} ; 1\right] .
$$

\footnotetext{
${ }^{3}$ This indexing is not to be confused with the indexing of edges described above.
} 
In appendix A we list some identities relating these Lauricella functions to other known functions.

Modulo the relation between Mellin and post-Mellin parameters which will be explained in section 2.1, this concludes the complete set of Feynman rules for writing down an explicit power series expansion of any scalar $n$-point conformal block with scalar exchanges in any channel.

Readers familiar with series expansions of conformal blocks may feel puzzled by the apparent cross-ratio-basis independence of the series coefficients appearing in the expansion (2.3). However, the explicit form of the edge and vertex factors does in fact depend on the choice of basis of cross-ratios; this dependence is encoded in the correct pairing between the single-trace parameters and cross-ratios as discussed above, as well as the precise relation between Mellin and post-Mellin parameters, which we discuss next. At the end, as noted in (2.3), one sums over all single-trace and Mellin parameters.

\subsection{Mellin and post-Mellin parameters}

Recall that Mellin variables [71, 72] are complex-valued variables $\gamma_{i j}(1 \leq i, j \leq n)$ which are symmetric, $\gamma_{i j}=\gamma_{j i}$ with $\gamma_{i i}:=-\Delta_{i}$, satisfying the following $n$ constraints:

$$
\sum_{j=1}^{n} \gamma_{i j}=0 \quad(i=1, \ldots, n) .
$$

This leads to $n(n-3) / 2$ independent components. These variables play a central role in the context of Mellin amplitudes of $n$-point bulk diagrams [68-70,73], which will be reviewed in section 4 in the proof of the proposed Feynman rules for conformal blocks. The constraints above can be solved in terms of auxiliary momentum variables $p_{i}$ (for $i=1, \ldots, n$ ) such that $p_{i} \cdot p_{j}:=\gamma_{i j}$ (thus individual $p_{i}$ are "on-shell," i.e. $p_{i} \cdot p_{i}=-\Delta_{i}$ ), when "momentum conservation," $\sum_{i=1}^{n} p_{i}=0$ is imposed. In this auxiliary space, the role of the $n(n-3) / 2$ independent Mellin variables is played by a choice of $n(n-3) / 2$ independent Mandelstam invariants $s_{i_{1} \ldots i_{k}}$ defined via

$$
s_{i_{1} \ldots i_{k}}:=-\left(p_{i_{1}}+\cdots+p_{i_{k}}\right)^{2}=\sum_{j=i_{1}}^{i_{k}} \Delta_{j}-2 \sum_{i_{1} \leq r<s \leq i_{k}} \gamma_{r s} .
$$

In section 4, for working out the Feynman rules, we will be interested in the following object which we call the "Mellin product,"

$$
\prod_{1 \leq i<j \leq n} \frac{1}{\left(x_{i j}^{2}\right)^{\gamma_{i j}}}
$$

where $x_{i}$ (for $i=1, \ldots, n$ ) are boundary coordinates at which operators of conformal dimension $\Delta_{i}$ are inserted. The reason why such an object appears will be clear in section 4 where we obtain the conformal block Feynman rules starting from the Mellin representation of certain bulk Witten diagrams. This product can be recast in terms of conformal crossratios built out of $x_{i}$ coordinates, as we now describe. 
For any given choice of independent cross-ratios and a given channel, there is a canonical choice of $n(n-3) / 2$ independent Mellin variables,

$$
\left\{\gamma_{i j}:(i j) \in \mathcal{U}_{\text {channel }}\right\} \bigcup\left\{\gamma_{i j}:(i j) \in \mathcal{V}_{\text {channel }}\right\},
$$

which makes the Mellin product expressed in terms of cross-ratios physically intuitive. The sets $\mathcal{U}_{\text {channel }}$ and $\mathcal{V}_{\text {channel }}$, of cardinalities $n-3$ and $\left(\begin{array}{c}n-2 \\ 2\end{array}\right)$ respectively, will be defined shortly. More precisely, given a particular channel and any choice of conformal crossratios $\left\{u_{i}, v_{r s}\right\}$ consistent with the discussion around (2.1)-(2.2), there exists a choice of independent Mellin variables $\gamma_{i j}$ (2.14) such that the Mellin product can be re-expressed in terms of a product over powers of the given cross-ratios, ${ }^{4}$

$$
\prod_{1 \leq i<j \leq n} \frac{1}{\left(x_{i j}^{2}\right)^{\gamma_{i j}}}=: W_{n}^{0}\left(x_{i}\right)\left(\prod_{i=1}^{n-3} u_{i}^{s_{i} / 2}\right)\left(\prod_{(r s) \in \mathcal{V}_{\text {channel }}} v_{r s}^{-\gamma_{r s}}\right),
$$

where $W_{n}^{0}$ is the leg-factor for the given choice of cross-ratios in the particular channel. The set $\left\{s_{i}\right\}$ is the set of $(n-3)$ independent Mandelstam invariants associated with the $(n-3)$ internal legs of the binary graph representation of the block. In enumerating the Mandelstam invariants, we labeled the internal edges with an index $i=1, \ldots, n-3$ such that the Mandelstam invariant for the edge $i$, given by $s_{i}$, appears in the exponent of the cross-ratio $u_{i}$. Accordingly, we can assign the single-trace parameter associated with this internal edge, appearing in the summand of (2.3), the edge factor (2.4) and the vertex factors $(2.6)$ to be $k_{i}$.

In the final product in (2.15), the set of Mellin variables appearing in the exponents determines precisely the set $\left\{\gamma_{i j}:(i j) \in \mathcal{V}_{\text {channel }}\right\}$. This will be taken to be the definition of $\mathcal{V}_{\text {channel. }}$. The set $\mathcal{U}_{\text {channel }}$ is then defined to be the set of pairs of indices such that $\left\{\gamma_{i j}\right.$ : $\left.(i j) \in \mathcal{U}_{\text {channel }}\right\}$ gives the residual $n-3$ independent Mellin variables. It is worth noting that dependence in (2.15) on the Mellin variables from this set is encoded in the Mandelstam invariants $s_{i}$. We will denote $\mathcal{D}_{\text {channel }}$ to be the set such that $\left\{\gamma_{i j}:(i j) \in \mathcal{D}_{\text {channel }}\right\}$ produces all dependent Mellin variables. Of course, the union of all these sets gives

$$
\mathcal{U}_{\text {channel }} \bigcup \mathcal{V}_{\text {channel }} \bigcup \mathcal{D}_{\text {channel }}=\{(i j): 1 \leq i<j \leq n\}
$$

We note that the sets $\mathcal{U}_{\text {channel }}, \mathcal{V}_{\text {channel }}$, and $\mathcal{D}_{\text {channel }}$ for a fixed topology are not unique but depend on the choice of input cross-ratios. Even for a fixed set of input cross-ratios, the choice of sets $\mathcal{U}_{\text {channel }}$ and $\mathcal{D}_{\text {channel }}$ is not unique.

We define the set of Mellin parameters to be the set

$$
\mathcal{J}_{\text {channel }}:=\left\{j_{r s}:(r s) \in \mathcal{V}_{\text {channel }}\right\},
$$

of cardinality $\left(\begin{array}{c}n-2 \\ 2\end{array}\right)$. Mellin parameters make a direct appearance in the summand of the Feynman prescription for conformal blocks (2.3), where they appear in the exponents of certain cross-ratios, as well as in the edge and vertex factors (2.4) and (2.6) via the post-Mellin

\footnotetext{
${ }^{4}$ Without loss of generality, we are assuming the given cross-ratios have been enumerated such that the subscripts match, i.e. $u_{i}$ goes with $s_{i}$ and $v_{r s}$ goes with $\gamma_{r s}$ as shown in $(2.15)$.
} 
parameters $\ell_{a}$. To obtain the full conformal block, one sums all Mellin parameters over all integral values from 0 to $\infty$. We now give the prescription to compute the post-Mellin parameters $\ell_{a}$ associated with the conformal dimensions $\Delta_{a}$ in terms of the Mellin parameters.

For an external operator with conformal dimension $\Delta_{i}=-\gamma_{i i}$ inserted at position $x_{i}$, we define the associated post-Mellin parameter to be

$$
\ell_{i}:=\sum_{\substack{(r s) \in \mathcal{V}_{\text {channel }} \\ r=i \text { or } s=i}} j_{r s}=\sum_{\substack{j_{r s} \in \mathcal{J}_{\text {channel }} r=i \text { or } s=i}} j_{r s} .
$$

If the set $\left\{(r s) \in \mathcal{V}_{\text {channel }}: r=i\right.$ or $\left.s=i\right\}$ is empty, then $\ell_{i}=0$. Note that this definition implies that the sum over all post-Mellin parameters associated to external conformal dimensions evaluates to twice the sum over all Mellin parameters,

$$
\sum_{i=1}^{n} \ell_{i}=2 \sum_{\mathcal{J}_{\text {channel }}} j_{r s}
$$

For exchanged operators of conformal dimensions $\Delta_{\delta_{i}}$, the prescription to compute the post-Mellin parameters proceeds iteratively as follows:

1. First, at all internal vertices of the binary graph with precisely two external edges and one internal edge incident, add the post-Mellin parameters associated with the external dimensions, and then drop all terms which are multiples of two (i.e. terms which are even for all integral values of the Mellin parameters). Assign this nonnegative sum to be the post-Mellin parameter of the internal (exchanged) operator.

$$
\bigvee_{\Delta_{\delta_{3}}}^{\Delta_{1}}: \ell_{\delta_{3}} \stackrel{2 \mathcal{J}}{=} \ell_{1}+\ell_{2},
$$

where the symbol $\stackrel{2 \mathcal{J}}{=}$ means equality holds once one drops all terms which are even for all integral values of Mellin parameters. For example, if $\ell_{1}=j_{12}+j_{13}+j_{16}$ and $\ell_{2}=j_{12}+j_{23}+j_{24}$, then $\ell_{\delta_{3}} \stackrel{2 \mathcal{J}}{=} \ell_{1}+\ell_{2}$ implies $\ell_{\delta_{3}}=j_{13}+j_{16}+j_{23}+j_{24}$.

2. If all internal post-Mellin parameters have not already been determined, pick any internal vertex where the post-Mellin parameters of precisely two of the edges are already known. The post-Mellin parameter of the third edge is given by the sum of the other two post-Mellin parameters, after dropping terms which are even multiples of Mellin parameters, exactly as shown in (2.20). For example, if two of the postMellin parameters are known at a vertex with three incident exchanged operators, then the third is determined as follows:

$$
\overbrace{\Delta_{\delta_{3}}}^{\Delta_{\delta_{1}} \Delta_{\delta_{2}}}: \ell_{\delta_{3}} \stackrel{2 \mathcal{J}}{=} \ell_{\delta_{1}}+\ell_{\delta_{2}} .
$$


If there are multiple choices of vertices for fixing the unknown post-Mellin parameter of an internal edge, pick any. The final assignment will be independent of this choice.

3. Repeat step 2 until all internal conformal dimensions have been assigned a post-Mellin parameter.

Note that this prescription guarantees that all post-Mellin parameters are written as positive linear combinations of Mellin parameters. Furthermore, at any internal vertex, the sum of any two of the post-Mellin parameters equals the third post-Mellin parameter up to terms which are even multiples of Mellin parameters. That is, if $\ell_{a}, \ell_{b}$ and $\ell_{c}$ are the post-Mellin parameters for conformal dimensions incident at a common vertex, then

$$
\ell_{a} \stackrel{2 \mathcal{J}}{=} \ell_{b}+\ell_{c} \quad \ell_{b} \stackrel{2 \mathcal{J}}{=} \ell_{c}+\ell_{a} \quad \ell_{c} \stackrel{2 \mathcal{J}}{=} \ell_{a}+\ell_{b} .
$$

In the next section, we illustrate how to apply these rules to determine the $n$-point conformal block in the comb channel, the $n$-point conformal block in the OPE channel, and the seven-point block in the mixed channel (all depicted in figure 1). In section 4, we will reproduce these blocks from first principles which serves as a highly non-trivial check of the Feynman rules.

\section{Examples}

In this section, we illustrate how to apply the Feynman rules to three classes of examples: the $n$-point conformal block in the comb channel and the OPE channel for arbitrary $n,{ }^{5}$ and the seven-point mixed channel block (see figure 1 for their definitions). All known $d$ dimensional scalar conformal blocks with scalar exchanges in the literature fall into one of the classes above. This includes the well-known four-point block, and the recently obtained five-point block [53], $n$-point comb channel blocks $[58,60]$ and the six-point OPE channel block [61]. However, the seven-point example to be discussed next and the $n$-point OPE channel examples for $n \geq 8$ are new results.

We invite the reader to test their understanding of section 2 by applying the Feynman rules in the trivial case of the four-point block and rediscover the well-known series expansion, or the slightly less non-trivial though straightforward case of the five-point block. These are special cases of the $n$-point comb channel block which is discussed in section 3.2.

\subsection{Seven-point mixed channel block}

In this section we work out the seven-point conformal block in the "mixed channel" shown in figure $1 \mathrm{~b}$. We use the following independent cross-ratios as input data:

$$
\begin{aligned}
u_{1} & :=\frac{x_{12}^{2} x_{37}^{2}}{x_{17}^{2} x_{23}^{2}} \quad u_{2}:=\frac{x_{23}^{2} x_{57}^{2}}{x_{25}^{2} x_{37}^{2}} \quad u_{3}:=\frac{x_{45}^{2} x_{27}^{2}}{x_{25}^{2} x_{47}^{2}} \quad u_{4}:=\frac{x_{67}^{2} x_{25}^{2}}{x_{27}^{2} x_{56}^{2}} \\
v_{13} & :=\frac{x_{13}^{2} x_{27}^{2}}{x_{17}^{2} x_{23}^{2}} \quad v_{i 6}:=\frac{x_{i 6}^{2} x_{57}^{2}}{x_{i 7}^{2} x_{56}^{2}} \quad(1 \leq i \leq 4) \\
v_{i j} & :=\frac{x_{i j}^{2} x_{27}^{2} x_{57}^{2}}{x_{i 7}^{2} x_{j 7}^{2} x_{25}^{2}} \quad((i j) \in\{(14),(15),(24),(34),(35)\}) .
\end{aligned}
$$

\footnotetext{
${ }^{5}$ We remind the reader that the OPE channel in this paper is only defined for even $n$.
} 
It is easily checked that they satisfy the conditions discussion around (2.1)-(2.2). Explicitly, keeping track of which cross-ratios vanish and which tend to unity as we take the following sequence of OPE limits in the given order, we note

$$
\begin{aligned}
& x_{2} \rightarrow x_{1}: u_{1} \rightarrow 0, v_{13} \rightarrow 1, v_{15} \rightarrow 1 \\
& x_{2} \rightarrow x_{3}: u_{2} \rightarrow 0, v_{35} \rightarrow 1 \\
& x_{4} \rightarrow x_{5}: u_{3} \rightarrow 0, v_{24} \rightarrow 1, v_{34} \rightarrow 1, v_{14} \rightarrow 1, v_{46} \rightarrow 1 \\
& x_{6} \rightarrow x_{7}: u_{4} \rightarrow 0, v_{16} \rightarrow 1, v_{26} \rightarrow 1, v_{36} \rightarrow 1,
\end{aligned}
$$

so that by the end all $u_{i} \rightarrow 0$ and all $v_{r s} \rightarrow 1$.

A convenient choice of dependent Mellin variables which turns the Mellin product (2.13) for the seven-point block in mixed channel into the form (2.15) is

$$
\left\{\gamma_{i j}:(i j) \in \mathcal{D}_{7, \text { mix }}\right\} \quad \text { where } \quad \mathcal{D}_{7, \text { mix }}=\{(17),(25),(27),(37),(47),(56),(57)\} .
$$

This choice is not unique and can be obtained by trial and error using the given cross-ratios. A different choice of set $\mathcal{D}_{7 \text {,mix }}$ will result in a different set $\mathcal{U}_{7 \text {,mix }}$ but it will not affect the set $\mathcal{V}_{7, \text { mix }}$ which is determined uniquely in terms of the input cross-ratios. In terms of the independent Mellin variables, these can be expressed as

$$
\begin{aligned}
& \gamma_{17}=\Delta_{1}-\gamma_{12}-\gamma_{13}-\gamma_{14}-\gamma_{15}-\gamma_{16} \\
& \gamma_{25}=\Delta_{12345,67}-\gamma_{12}-\gamma_{13}-\gamma_{14}-\gamma_{15}-\gamma_{23}-\gamma_{24}-\gamma_{34}-\gamma_{35}-\gamma_{45}+\gamma_{67} \\
& \gamma_{27}=\Delta_{267,1345}+\gamma_{13}+\gamma_{14}+\gamma_{15}-\gamma_{26}+\gamma_{34}+\gamma_{35}+\gamma_{45}-\gamma_{67} \\
& \gamma_{37}=\Delta_{3}-\gamma_{13}-\gamma_{23}-\gamma_{34}-\gamma_{35}-\gamma_{36} \\
& \gamma_{47}=\Delta_{4}-\gamma_{14}-\gamma_{24}-\gamma_{34}-\gamma_{45}-\gamma_{46} \\
& \gamma_{56}=\Delta_{6}-\gamma_{16}-\gamma_{26}-\gamma_{36}-\gamma_{46}-\gamma_{67} \\
& \gamma_{57}=\Delta_{57,12346}+\gamma_{12}+\gamma_{13}+\gamma_{14}+\gamma_{16}+\gamma_{23}+\gamma_{24}+\gamma_{26}+\gamma_{34}+\gamma_{36}+\gamma_{46} .
\end{aligned}
$$

In terms of these, the Mellin product then takes the form

$$
\prod_{1 \leq i<j \leq 7} \frac{1}{\left(x_{i j}^{2}\right)^{\gamma_{i j}}}=: W_{7, \text { mix }}^{0}\left(x_{i}\right)\left(\prod_{i=1}^{4} u_{i}^{s_{i} / 2}\right)\left(\prod_{(r s) \in \mathcal{V}_{7, \text { mix }}} v_{r s}^{-\gamma_{r s}}\right),
$$

where the leg-factor turns can be expressed as

$$
\begin{aligned}
W_{7, \text { mix }}^{0}= & \left(\frac{x_{27}^{2}}{x_{12}^{2} x_{17}^{2}}\right)^{\frac{\Delta_{1}}{2}}\left(\frac{x_{17}^{2}}{x_{12}^{2} x_{27}^{2}}\right)^{\frac{\Delta_{2}}{2}}\left(\frac{x_{27}^{2}}{x_{23}^{2} x_{37}^{2}}\right)^{\frac{\Delta_{3}}{2}}\left(\frac{x_{57}^{2}}{x_{45}^{2} x_{47}^{2}}\right)^{\frac{\Delta_{4}}{2}} \\
& \times\left(\frac{x_{47}^{2}}{x_{45}^{2} x_{57}^{2}}\right)^{\frac{\Delta_{5}}{2}}\left(\frac{x_{57}^{2}}{x_{56}^{2} x_{67}^{2}}\right)^{\frac{\Delta_{6}}{2}}\left(\frac{x_{56}^{2}}{x_{57}^{2} x_{67}^{2}}\right)^{\frac{\Delta_{7}}{2}}
\end{aligned}
$$

and the set associated to the Mellin parameters over which the $\left(\begin{array}{l}5 \\ 2\end{array}\right)$-dimensional product runs in (3.5) is,

$$
\mathcal{V}_{7, \text { mix }}=\{(13),(14),(15),(16),(24),(26),(34),(35),(36),(46)\} .
$$




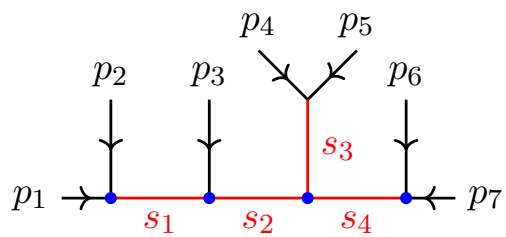

Figure 2. Auxiliary momenta and Mandelstam variable assignments: graphical representation of the 7-point conformal block in the mixed channel from figure $1 \mathrm{~b}$ with auxiliary momenta and Mandelstam variables shown.

The exponents $s_{i}$ in (3.5) are given by

$$
\begin{array}{ll}
s_{1}=\Delta_{1}+\Delta_{2}-2 \gamma_{12} & s_{2}=\Delta_{1}+\Delta_{2}+\Delta_{3}-2 \gamma_{12}-2 \gamma_{13}-2 \gamma_{23} \\
s_{3}=\Delta_{4}+\Delta_{5}-2 \gamma_{45} & s_{4}=\Delta_{6}+\Delta_{7}-2 \gamma_{67}
\end{array}
$$

which are indeed the Mandelstam invariants attached to the internal edges of the associated binary graph, as we now describe. In the auxiliary momentum space, one assigns an incoming momentum to each external edge of the unrooted binary tree representation of the conformal block, such that the sum over all momenta is zero (see figure 2). Let $p_{i}$ be the momentum attached to the external edge labelled with conformal dimension $\Delta_{i}$, with the on-shell condition $p_{i}^{2}=-\Delta_{i}$ and momentum conservation. Then the Mandelstam invariants associated to each internal leg are

$$
\begin{array}{ll}
s_{1}=-\left(p_{1}+p_{2}\right)^{2} & s_{2}=-\left(p_{1}+p_{2}+p_{3}\right)^{2} \\
s_{3}=-\left(p_{4}+p_{5}\right)^{2} & s_{4}=-\left(p_{6}+p_{7}\right)^{2} .
\end{array}
$$

Using (2.12), it is easy to see this gives back (3.8).

With the cross-ratios and the set $\mathcal{V}_{7 \text {,mixed }}$ in place, the only computational task remaining is determining the post-Mellin parameters. Recall that the Mellin parameters form the set (2.17) and the post-Mellin parameters for the external conformal dimensions/edges are given by (2.18). Explicitly, for the present choice of cross-ratios, this yields

$$
\begin{aligned}
& \ell_{1}=j_{13}+j_{14}+j_{15}+j_{16} \quad \ell_{2}=j_{24}+j_{26} \\
& \ell_{3}=j_{13}+j_{34}+j_{35}+j_{36} \quad \ell_{4}=j_{14}+j_{24}+j_{34}+j_{46} \\
& \ell_{5}=j_{15}+j_{35} \quad \ell_{6}=j_{16}+j_{26}+j_{36}+j_{46} \quad \ell_{7}=0 .
\end{aligned}
$$

Now one can solve for the post-Mellin parameters for the internal edges/exchanged dimensions using the algorithm described around (2.20)-(2.21). For illustrative purposes, we work it out explicitly for each internal leg below. 
1. First we consider all vertices with precisely two incident external edges and one incident internal edge:

$$
\bigvee_{\Delta_{\delta_{1}}}^{\Delta_{1}}: \ell_{\delta_{1}} \stackrel{2 \mathcal{J}}{=} \ell_{1}+\ell_{2} \Rightarrow \ell_{\delta_{1}}=j_{13}+j_{14}+j_{15}+j_{16}+j_{24}+j_{26}
$$$$
\bigvee_{\Delta_{\delta_{3}}}^{\Delta_{4}}: \ell_{\delta_{3}} \stackrel{2 \mathcal{J}}{=} \ell_{4}+\ell_{5} \Rightarrow \ell_{\delta_{3}}=j_{14}+j_{15}+j_{24}+j_{34}+j_{35}+j_{46}
$$$$
\bigvee_{\Delta_{\delta_{4}}}^{\Delta_{6}}: \ell_{\delta_{4}} \stackrel{2 \mathcal{J}}{=} \ell_{6}+\ell_{7} \Rightarrow \ell_{\delta_{4}}=j_{16}+j_{26}+j_{36}+j_{46} .
$$

2. Finally, to determine $\ell_{\delta_{2}}$, one can choose to look at one of two possible vertices. We will work it out using both to demonstrate choice-independence. From one choice of a vertex, we get

$$
\bigvee^{\Delta_{3}}: \quad \ell_{\delta_{2}} \stackrel{2 \mathcal{J}}{=} \ell_{3}+\ell_{\delta_{1}} \Rightarrow \ell_{\delta_{2}}=j_{14}+j_{15}+j_{16}+j_{24}+j_{26}+j_{34}+j_{35}+j_{36}
$$

On the other hand, the choosing the following vertex yields,

$$
\overbrace{\Delta_{\delta_{2}}}^{\Delta_{\delta_{3}} \Delta_{\delta_{4}}}: \quad \ell_{\delta_{2}} \stackrel{2 \mathcal{J}}{=} \ell_{\delta_{3}}+\ell_{\delta_{4}} \Rightarrow \ell_{\delta_{2}}=j_{14}+j_{15}+j_{16}+j_{24}+j_{26}+j_{34}+j_{35}+j_{36}
$$

As promised, the assignments agree.

Now, using (2.4) and (2.6), we can write down the internal edge and vertex factors for the conformal block. As described in section 2.1, to each internal edge associated with the Mandelstam invariant $s_{i}$ (see figure 2 and equation (3.8)), assign the single-trace parameter 
$k_{i}$. Then, the $(7-3=4)$ edge factors are

$$
\begin{aligned}
& \bullet \frac{k_{1}}{\Delta_{\delta_{1}}} \cdot E_{1}=\frac{\left(\Delta_{\delta_{1}}-h+1\right)_{k_{1}}}{\left(\Delta_{\delta_{1}}\right)_{2 k_{1}+j_{13}+j_{14}+j_{15}+j_{16}+j_{24}+j_{26}}} \\
& \bullet \frac{k_{2}}{\Delta_{\delta_{2}}} \cdot E_{2}=\frac{\left(\Delta_{\delta_{1}}-h+1\right)_{k_{2}}}{\left(\Delta_{\delta_{2}}\right)_{2 k_{2}+j_{14}+j_{15}+j_{16}+j_{24}+j_{26}+j_{34}+j_{35}+j_{36}}} \\
& -\frac{k_{3}}{\Delta_{\delta_{3}}} \cdot: E_{3}=\frac{\left(\Delta_{\delta_{1}}-h+1\right)_{k_{3}}}{\left(\Delta_{\delta_{3}}\right)_{2 k_{3}+j_{14}+j_{15}+j_{24}+j_{34}+j_{35}+j_{46}}} \\
& -\frac{k_{4}}{\Delta_{\delta_{4}}} \cdot E_{4}=\frac{\left(\Delta_{\delta_{4}}-h+1\right)_{k_{4}}}{\left(\Delta_{\delta_{4}}\right)_{2 k_{4}+j_{16}+j_{26}+j_{36}+j_{46}}},
\end{aligned}
$$

and the $(7-2=5)$ internal vertices of the unrooted binary tree, listed here:

$$
V_{1}: \bigvee_{k_{1}}^{\Delta_{1}} V_{2}: \underbrace{\Delta_{2}}_{k_{3}} V_{3}: \underbrace{\Delta_{\delta_{3}}}_{\delta_{\delta_{1}}}
$$

give the following vertex factors:

$$
\begin{aligned}
& V_{1}=\left(\Delta_{12, \delta_{1}}\right)_{-k_{1}}\left(\Delta_{2 \delta_{1}, 1}\right)_{k_{1}+j_{24}+j_{26}}\left(\Delta_{1 \delta_{1}, 2}\right)_{k_{1}+j_{13}+j_{14}+j_{15}+j_{16}} \\
& \times F_{A}^{(1)}\left[\Delta_{12 \delta_{1}},-h ;\left\{-k_{1}\right\} ;\left\{\Delta_{\delta_{1}}-h+1\right\} ; 1\right] \\
& V_{2}=\left(\Delta_{45, \delta_{3}}\right)_{-k_{3}}\left(\Delta_{5 \delta_{3}, 4}\right)_{k_{3}+j_{15}+j_{35}}\left(\Delta_{4 \delta_{3}, 5}\right)_{k_{3}+j_{14}+j_{24}+j_{34}+j_{46}} \\
& \times F_{A}^{(1)}\left[\Delta_{45 \delta_{3}},-h ;\left\{-k_{3}\right\} ;\left\{\Delta_{\delta_{3}}-h+1\right\} ; 1\right] \\
& V_{3}=\left(\Delta_{67, \delta_{4}}\right)_{-k_{4}}\left(\Delta_{7 \delta_{4}, 6}\right)_{k_{4}}\left(\Delta_{6 \delta_{4}, 7}\right)_{k_{4}+j_{16}+j_{26}+j_{36}+j_{46}} \\
& \times F_{A}^{(1)}\left[\Delta_{67 \delta_{4}},-h ;\left\{-k_{4}\right\} ;\left\{\Delta_{\delta_{4}}-h+1\right\} ; 1\right] \\
& V_{4}=\left(\Delta_{3 \delta_{1}, \delta_{2}}\right)_{k_{1}-k_{2}+j_{13}}\left(\Delta_{3 \delta_{2}, \delta_{1}}\right)_{k_{2}-k_{1}+j_{34}+j_{35}+j_{36}}\left(\Delta_{\delta_{1} \delta_{2}, 3}\right)_{k_{1}+k_{2}+j_{14}+j_{15}+j_{16}+j_{24}+j_{26}} \\
& \times F_{A}^{(2)}\left[\Delta_{3 \delta_{1} \delta_{2}},-h ;\left\{-k_{1},-k_{2}\right\} ;\left\{\Delta_{\delta_{1}}-h+1, \Delta_{\delta_{2}}-h+1\right\} ; 1,1\right] \\
& V_{5}=\left(\Delta_{\delta_{3} \delta_{4}, \delta_{2}}\right)_{k_{3}+k_{4}-k_{2}+j_{46}}\left(\Delta_{\delta_{2} \delta_{4}, \delta_{3}}\right)_{k_{2}+k_{4}-k_{3}+j_{16}+j_{26}+j_{36}} \\
& \times\left(\Delta_{\delta_{2} \delta_{3}, \delta_{4}}\right)_{k_{2}+k_{3}-k_{4}+j_{14}+j_{15}+j_{24}+j_{34}+j_{35}} \\
& \times F_{A}^{(3)}\left[\Delta_{\delta_{2} \delta_{3} \delta_{4}},-h ;\left\{-k_{2},-k_{3},-k_{4}\right\} ;\left\{\Delta_{\delta_{2}}-h+1, \Delta_{\delta_{3}}-h+1, \Delta_{\delta_{4}}-h+1\right\} ; 1,1,1\right] .
\end{aligned}
$$

The Lauricella functions appearing above can be simplified further into combinations of Pochhammer symbols and generalized hypergeometric functions (see appendix A). The 
final expression for the conformal block is given by (2.2)-(2.3) with the cross-ratios, edge and vertex factors as determined above.

In section 4.1 we will reproduce the seven-point mixed-channel conformal block of this section using holographic techniques which will involve the Mellin amplitude of a particular seven-point Witten diagram as the starting point.

\section{$3.2 \quad n$-point comb channel block}

In this section we will illustrate how to apply the Feynman rules to reproduce the $n$-point comb channel conformal block (see figure 1a) of ref. [58]. The first step involves picking the cross-ratios; in this section, we choose those from ref. [58], ${ }^{6}$

$$
u_{i}:=\frac{x_{1(i+1)}^{2} x_{(i+2) n}^{2}}{x_{1(i+2)}^{2} x_{(i+1) n}^{2}} \quad 1 \leq i \leq n-3, \quad v_{r s}:=\frac{x_{1 n}^{2} x_{r s}^{2}}{x_{1 s}^{2} x_{r n}^{2}} \quad 2 \leq r<s \leq n-1 .
$$

It turns out, the associated canonical choice of $n$ dependent Mellin variables is given by the set

$$
\left\{\gamma_{i j}:(i j) \in \mathcal{D}_{\text {comb }}\right\} \quad \text { where } \quad \mathcal{D}_{\text {comb }}:=\{(j n): 1 \leq j \leq n-1\} \bigcup\{(1(n-1))\} .
$$

Explicitly, the dependent variables take the form, ${ }^{7}$

$$
\begin{aligned}
\gamma_{1(n-1)} & =\Delta_{12 \ldots(n-1), n}-\sum_{j=2}^{n-2} \gamma_{j(n-1)}-\sum_{j=2}^{n-2} \gamma_{1 j}-\sum_{2 \leq i<j \leq n-2} \gamma_{i j} \\
\gamma_{1 n} & =\Delta_{1 n, 23 \ldots(n-1)}+\sum_{j=2}^{n-2} \gamma_{j(n-1)}+\sum_{2 \leq i<j \leq n-2} \gamma_{i j} \\
\gamma_{i n} & =-\sum_{j=1}^{n-1} \gamma_{i j} \quad(2 \leq i \leq n-2) \\
\gamma_{(n-1) n} & =\Delta_{(n-1) n, 12 \ldots(n-2)}+\sum_{j=2}^{n-2} \gamma_{1 j}+\sum_{2 \leq i<j \leq n-2} \gamma_{i j},
\end{aligned}
$$

which, along with $\gamma_{i j}=\gamma_{j i}$, and $\gamma_{i i}=-\Delta_{i}$ for all $i, j$ explicitly solves (2.11) as required.

After substituting in (3.19), the Mellin product, expressed in terms of the crossratios (3.17) becomes

$$
\prod_{1 \leq i<j \leq n} \frac{1}{\left(x_{i j}^{2}\right)^{\gamma_{i j}}}=: W_{n, \mathrm{comb}}^{0}\left(x_{i}\right)\left(\prod_{i=1}^{n-3} u_{i}^{s_{i} / 2}\right)\left(\prod_{2 \leq r<s \leq n-1} v_{r s}^{-\gamma_{r s}}\right),
$$

\footnotetext{
${ }^{6}$ In ref. [60] the authors obtained an alternate expression for the conformal block based on a different choice of cross-ratios than the one used in ref. [58]. Nevertheless these different forms are expected to be equivalent. As noted previously, one of the places the dependence on the choice of cross-ratios shows up in the series expansion is in the precise post-Mellin parameters appearing in the Pochhammer symbol. Moreover, in ref. [53] the author used a different set of cross-ratios for the five-point block. The proposed Feynman rules applied to this choice of cross-ratios readily reproduces the block obtained there.

${ }^{7}$ For a different choice of basis of cross-ratios, there will accordingly be a different canonical basis of independent Mellin variables.
} 


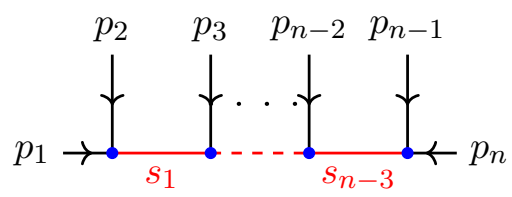

Figure 3. Auxiliary momenta and Mandelstam variable assignments: graphical representation of $n$-point conformal blocks in the comb and channel from figure 1a with auxiliary momenta and Mandelstam variables shown.

where the leg-factor $W_{n, \text { comb }}^{0}$ is given by

$$
W_{n, \mathrm{comb}}^{0}\left(x_{i}\right)=\left(\frac{x_{2 n}^{2}}{x_{1 n}^{2} x_{12}^{2}}\right)^{\frac{\Delta_{1}}{2}}\left(\frac{x_{1(n-1)}^{2}}{x_{1 n}^{2} x_{(n-1) n}^{2}}\right)^{\frac{\Delta_{n}}{2}} \prod_{i=2}^{n-1}\left(\frac{x_{1 n}^{2}}{x_{1 i}^{2} x_{i n}^{2}}\right)^{\frac{\Delta_{i}}{2}}
$$

and the $s_{i}$ are expressible as,

$$
s_{i}=\sum_{j=1}^{i+1} \Delta_{j}-2 \sum_{1 \leq r<s \leq i+1} \gamma_{r s} \quad 1 \leq i \leq n-3 .
$$

The set in (3.18) is the canonical choice of dependent Mellin variables precisely because it leads directly to (3.20).

As desired, the $s_{i}$ are the $n-3$ Mandelstam invariants associated with the $n-3$ internal edges. To see this, pass again to the auxiliary momentum space, and assign an incoming momentum to each external edge of the unrooted binary tree representation of the conformal block, such that the sum over all momenta is zero. Let $p_{i}$ be the momentum attached to the external edge labelled with conformal dimension $\Delta_{i}$, with the on-shell condition $p_{i}^{2}=-\Delta_{i}$ and momentum conservation, with the identification $\gamma_{i j}:=p_{i} \cdot p_{j}$. Then the Mandelstam invariants associated to each internal leg are (see figure 3)

$$
s_{i}:=-\left(p_{1}+\cdots+p_{i+1}\right)^{2} \quad 1 \leq i \leq n-3,
$$

which precisely evaluates to (3.22). Additionally, as described in section 2.1, to each internal edge with Mandelstam invariant $s_{i}$, we also assign the single-trace parameter $k_{i}$.

Furthermore, from (3.20) we also identify the index set

$$
\mathcal{V}_{\text {comb }}:=\{(r s): 2 \leq r<s \leq n-1\}
$$

which leads directly to the set of Mellin parameters (2.17). This, in turn, allows us to determine the post-Mellin parameters in terms of Mellin parameters. First, let's work out 
the post-Mellin parameters associated to external dimensions/external edges (2.18): ${ }^{8}$

$$
\begin{aligned}
\ell_{1} & =0 & \ell_{n} & =0 \\
\ell_{k} & =\sum_{r=2}^{k-1} j_{r k}+\sum_{s=k+1}^{n-1} j_{k s} & & (2 \leq k \leq n-1) .
\end{aligned}
$$

Next, applying (2.20) to the internal vertices at either extremes of the comb channel, we obtain the post-Mellin parameters $\ell_{\delta_{1}}$ and $\ell_{\delta_{n-3}}$ :

$$
\underbrace{\Delta_{1}}_{\Delta_{\delta_{1}}}: \ell_{\delta_{1}} \stackrel{2 \mathcal{J}}{=} \ell_{1}+\ell_{2} \quad \Rightarrow \ell_{\delta_{1}}=\ell_{2}=\sum_{s=3}^{n-1} j_{2 s}
$$

Finally to determine the remaining post-Mellin parameters, we use (2.21) on vertices with two internal edges and one external edge attached. For example, one can start with the vertex:

$$
\bigcap_{\Delta_{\delta_{2}}}^{\Delta_{\delta_{1}} \Delta_{3}}: \ell_{\delta_{2}} \stackrel{2 \mathcal{J}}{=} \ell_{\delta_{1}}+\ell_{3} \quad \Rightarrow \ell_{\delta_{2}}=\sum_{s=4}^{n-1} j_{2 s}+\sum_{s=4}^{n-1} j_{3 s},
$$

and then proceed one vertex to the right:

$$
\bigvee_{\Delta_{\delta_{3}}}^{\Delta_{\delta_{2}}}: \ell_{\delta_{3}} \stackrel{2 \mathcal{J}}{=} \ell_{\delta_{2}}+\ell_{4} \quad \Rightarrow \ell_{\delta_{3}}=\sum_{s=5}^{n-1} j_{2 s}+\sum_{s=5}^{n-1} j_{3 s}+\sum_{s=5}^{n-1} j_{4 s},
$$

and so on. Proceeding iteratively, we find

$$
\ell_{\delta_{i}}=\sum_{r=2}^{i+1} \sum_{s=i+2}^{n-1} j_{r s} \quad(1 \leq i \leq n-3),
$$

where the results from (3.27) have been included in the formula above.

$$
\begin{aligned}
& { }^{8} \text { For example, for } n \geq 8 \\
& \begin{array}{llll}
\ell_{2}=\sum_{s=3}^{n-1} j_{2 s} & \ell_{3}=j_{23}+\sum_{s=4}^{n-1} j_{3 s} & \ell_{4}=\sum_{r=2}^{3} j_{r 4}+\sum_{s=5}^{n-1} j_{4 s} \\
\ell_{5}=\sum_{r=2}^{4} j_{r 5}+\sum_{s=6}^{n-1} j_{5 s} & \ell_{6}=\sum_{r=2}^{5} j_{r 6}+\sum_{s=7}^{n-1} j_{6 s} & \ell_{7}=\sum_{r=2}^{6} j_{r 7}+\sum_{s=8}^{n-1} j_{7 s} .
\end{array}
\end{aligned}
$$


This is the full extent of computations needed to write down the $n$-point comb channel conformal block. The final step involves substituting the single-trace and post-Mellin parameters into the edge (2.4) and vertex (2.6) factors, which immediately yields the explicit conformal block via (2.3).

For the sake of completeness, we provide the explicit edge and vertex factors below. The $(n-3)$ edge factors are:

$$
-\frac{k_{i}}{\Delta_{\delta_{i}}}: E_{i}=\frac{\left(\Delta_{\delta_{i}}-h+1\right)_{k_{i}}}{\left(\Delta_{\delta_{i}}\right)_{2 k_{i}+\sum_{r=2}^{i+1} \sum_{s=i+2}^{n-1} j_{r s}}} \quad(1 \leq i \leq n-3) .
$$

Similarly, for the vertex factors we simply substitute all the ingredients from above into (2.6). To facilitate comparison with the result from ref. [58] (as well as the new derivation in section 4.2) we will simplify the linear combination of post-Mellin parameters appearing in the vertex factors. Rewriting,

$\ell_{\delta_{i}}=\sum_{r=2}^{i+1} \sum_{s=i+3}^{n-1} j_{r s}+\sum_{r=2}^{i+1} j_{r(i+2)} \quad \ell_{\delta_{i+1}}=\sum_{r=2}^{i+1} \sum_{s=i+3}^{n-1} j_{r s}+\sum_{s=i+3}^{n-1} j_{(i+2) s} \quad \ell_{i+2}=\sum_{r=2}^{i+1} j_{r(i+2)}+\sum_{s=i+3}^{n-1} j_{(i+2) s}$

for $0 \leq i \leq n-3$ where we used the identifications $\Delta_{\delta_{0}}:=\Delta_{1}$ and $\Delta_{\delta_{n-2}}:=\Delta_{n}$, simple arithmetic leads to

$$
\frac{1}{2} \ell_{(i+2) \delta_{i}, \delta_{i+1}}=\sum_{r=2}^{i+1} j_{r(i+2)} \quad \frac{1}{2} \ell_{\delta_{i} \delta_{i+1},(i+2)}=\sum_{r=2}^{i+1} \sum_{s=i+3}^{n-1} j_{r s} \quad \frac{1}{2} \ell_{(i+2) \delta_{i+1}, \delta_{i}}=\sum_{s=i+3}^{n-1} j_{(i+2) s}
$$

for $0 \leq i \leq n-3$. Then the $n-2$ internal vertices,

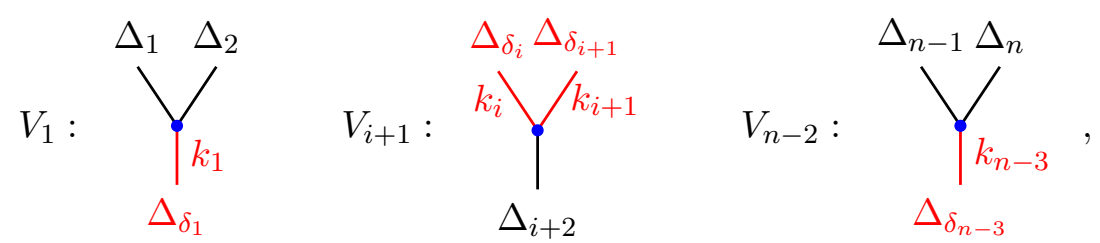

for $1 \leq i \leq n-4$, are associated with the vertex factors

$$
\begin{aligned}
V_{1}= & \left(\Delta_{12, \delta_{1}}\right)_{-k_{1}}\left(\Delta_{\delta_{1} 1,2}\right)_{k_{1}}\left(\Delta_{\delta_{1} 2,1}\right)_{k_{1}+\sum_{s=3}^{n-1} j_{2 s}} \\
& \times F_{A}^{(1)}\left[\Delta_{12 \delta_{1}},-h ;\left\{-k_{1}\right\} ;\left\{\Delta_{\delta_{1}}-h+1\right\} ; 1\right] \\
V_{i+1}:= & \left(\Delta_{\delta_{i} \delta_{i+1}, i+2}\right)_{k_{i(i+1)},}+\sum_{r=2}^{i+1} \sum_{s=i+3}^{n-1} j_{r s}\left(\Delta_{(i+2) \delta_{i}, \delta_{i+1}}\right)_{k_{i, i+1}+\sum_{r=2}^{i+1} j_{r(i+2)}} \\
& \times\left(\Delta_{(i+2) \delta_{i+1}, \delta_{i}}\right)_{k_{i+1, i}+\sum_{s=i+3}^{n-1} j_{(i+2) s}} \\
& \times F_{A}^{(2)}\left[\Delta_{\delta_{i} \delta_{i+1}(i+2)}-h ;\left\{-k_{i},-k_{i+1}\right\} ;\left\{\Delta_{\delta_{i}}-h+1, \Delta_{\delta_{i+1}}-h+1\right\} ; 1,1\right] \\
V_{n-2}= & \left(\Delta_{(n-1) n, \delta_{n-3}}\right)_{-k_{n-3}}\left(\Delta_{\delta_{n-3}(n-1), n}\right)_{k_{n-3}+\sum_{r=2}^{n-2} j_{r(n-1)}}\left(\Delta_{\delta_{n-3} n, n-1}\right)_{k_{n-3}} \\
& \times F_{A}^{(1)}\left[\Delta_{(n-1) n \delta_{n-3}},-h ;\left\{-k_{n-3}\right\} ;\left\{\Delta_{\delta_{n-3}}-h+1\right\} ; 1\right] .
\end{aligned}
$$

One can re-express the Lauricella functions of one and two variables above in terms of Pochhammer symbols and the generalized hypergeometric function ${ }_{3} F_{2}$, respectively 
(see (A.2)-(A.3)). Upon doing so, the final conformal block given by substituting the edge and vertex factors above into (2.2)-(2.3) finds precise agreement with the result of ref. [58].

Furthermore, one can check this reproduces the well-known four-point block upon setting $n=4$. Finally, ref. [53] worked out the $n=5$ block for a different set of crossratios. Starting with those cross-ratios as the input data, we checked that the Feynman rules reproduced precisely the block of ref. [53]. Generally, blocks from different choices of cross-ratios, though perhaps not manifestly identical, are still equivalent in the shared domain of convergence. In particular it can be checked that the five-point blocks of ref. [58], ref. [60] and ref. [53] are equivalent, even though they seem slightly different.

\section{$3.3 n$-point OPE channel block}

In the OPE channel (see figure 1c), we choose to represent the $n$-point conformal block (for even $n \geq 6$ ) in terms of the following cross-ratios: ${ }^{9}$

$$
\begin{array}{rlrl}
u_{1} & :=\frac{x_{12}^{2} x_{4 n}^{2}}{x_{1 n}^{2} x_{24}^{2}} & u_{n-3} & :=\frac{x_{(n-1) n}^{2} x_{2(n-2)}^{2}}{x_{(n-2)(n-1)}^{2} x_{2 n}^{2}} \\
u_{2 \times j} & :=\frac{x_{(2 j+1)(2 j+2)}^{2} x_{2 n}^{2}}{x_{(2 j+1) n}^{2} x_{2(2 j+2)}^{2}} \quad\left(1 \leq j \leq \frac{n}{2}-2\right) & u_{2 \times j-1}:=\frac{x_{2(2 j)}^{2} x_{(2 j+2) n}^{2}}{x_{(2 j) n}^{2} x_{2(2 j+2)}^{2}} \quad\left(2 \leq j \leq \frac{n}{2}-2\right) \\
v_{i(n-1)} & :=\frac{x_{i(n-1)}^{2} x_{(n-2) n}^{2}}{x_{i n}^{2} x_{(n-2)(n-1)}^{2}} \quad(1 \leq i \leq n-3) \\
v_{i j} & :=\frac{x_{i j}^{2} x_{2 n}^{2} x_{(2 t) n}^{2}}{x_{i n}^{2} x_{j n}^{2} x_{2(2 t)}^{2}} \quad\left(2 \leq t \leq \frac{n}{2}-1, \text { with } 1 \leq i \leq 2 t-2,2 t-1 \leq j \leq 2 t\right) .
\end{array}
$$

A convenient choice of dependent Mellin variables associated with the choice of crossratios above is given by the following index set

$$
\mathcal{D}_{\mathrm{OPE}}:=\{(i n) \mid 1 \leq i \leq n-2\} \bigcup\{((n-2)(n-1)),(2(n-2))\} .
$$

\footnotetext{
${ }^{9}$ Note that for certain choices of $(i j)$, the cross-ratio $v_{i j}$ in (3.35) identically evaluates to unity, in which case it is not to be included as an independent cross-ratio. The counting leading to $n(n-3) / 2$ independent cross-ratios accounts for such occurrences.
} 


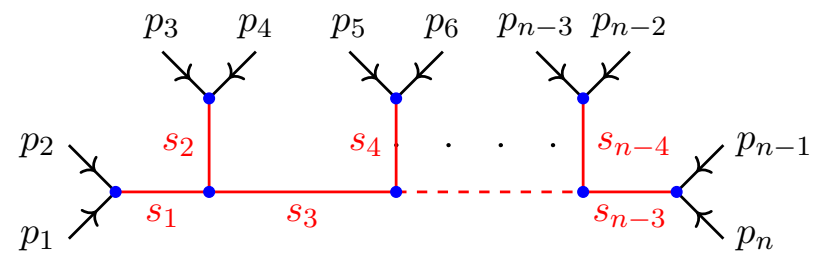

Figure 4. Auxiliary momenta and Mandelstam variable assignments: graphical representation of $n$ point conformal blocks in the OPE channel from figure 1c with auxiliary momenta and Mandelstam variables shown.

The dependent Mellin variables take the form

$$
\begin{aligned}
\gamma_{i n} & =-\sum_{j=1}^{n-1} \gamma_{i j} \quad(i=1,3,4,5,6, \ldots, n-4, n-3) \\
\gamma_{2 n} & =\Delta_{2(n-1) n, 134 \ldots(n-2)}-\gamma_{(n-1) n}-\gamma_{2(n-1)}+\sum_{j=3}^{n-2} \gamma_{1 j}+\sum_{3 \leq i<j \leq(n-2)} \gamma_{i j} \\
\gamma_{(n-2) n} & =\Delta_{(n-2) n, 123 \ldots(n-3)(n-1)}+\sum_{j=1}^{n-3} \gamma_{j(n-1)}+\sum_{1 \leq i<j \leq n-3} \gamma_{i j} \\
\gamma_{(n-2)(n-1)} & =-\sum_{\substack{j=1 \\
j \neq n-2}}^{n} \gamma_{j(n-1)} \\
\gamma_{2(n-2)} & =\Delta_{12 \ldots(n-2),(n-1) n}+\gamma_{(n-1) n}-\sum_{j=2}^{n-2} \gamma_{1 j}-\sum_{j=3}^{n-3} \gamma_{2 j}-\sum_{3 \leq i<j \leq n-2} \gamma_{i j} .
\end{aligned}
$$

Just like for the comb channel, it is useful to consider the auxiliary momentum space in the OPE channel. We use the convention for momentum and Mandelstam invariant assignments as depicted in figure 4. In this convention, the Mandelstam invariants associated with the internal legs take the following explicit form in terms of the independent Mellin variables:

$$
\begin{array}{rlrl}
s_{1} & :=-\left(p_{1}+p_{2}\right)^{2}=\Delta_{1}+\Delta_{2}-2 \gamma_{12} & \\
s_{n-3} & :=-\left(p_{n-1}+p_{n}\right)^{2}=\Delta_{n-1}+\Delta_{n}-2 \gamma_{(n-1) n} & & \left(1 \leq j \leq \frac{n}{2}-2\right) \\
s_{2 j} & :=-\left(p_{2 j+1}+p_{2 j+2}\right)^{2}=\Delta_{2 j+1}+\Delta_{2 j+2}-2 \gamma_{(2 j+1)(2 j+2)} & & (138) \\
s_{2 j-1} & :=-\left(p_{1}+p_{2}+p_{3}+\cdots+p_{2 j}\right)^{2}=\sum_{k=1}^{2 j} \Delta_{k}-2 \sum_{1 \leq r<s \leq 2 j} \gamma_{r s} \quad\left(2 \leq j \leq \frac{n}{2}-2\right) .
\end{array}
$$

With this in hand, it is straightforward to re-express the Mellin product (2.13) in terms of conformal cross-ratios (3.35):

$$
\prod_{1 \leq i<j \leq n} \frac{1}{\left(x_{i j}^{2}\right)^{\gamma_{i j}}}=W_{n, \mathrm{OPE}}^{0}\left(x_{i}\right)\left(\prod_{i=1}^{n-3} u_{i}^{s_{i} / 2}\right)\left(\prod_{(r s) \in \mathcal{V}_{\mathrm{OPE}}} v_{r s}^{-\gamma_{r s}}\right),
$$


where the leg-factor $W_{n, \mathrm{OPE}}^{0}$ is defined to be

$$
\begin{aligned}
W_{n, \mathrm{OPE}}^{0}\left(x_{i}\right):= & \left(\frac{x_{(n-2) n}^{2}}{x_{(n-2)(n-1)}^{2} x_{(n-1) n}^{2}}\right)^{\frac{\Delta_{n-1}}{2}}\left(\frac{x_{(n-2)(n-1)}^{2}}{x_{(n-2) n}^{2} x_{(n-1) n}^{2}}\right)^{\frac{\Delta_{n}}{2}} \\
& \times\left(\prod_{i=1}^{\frac{n}{2}-1}\left(\frac{x_{(2 i-1) n}^{2}}{x_{(2 i-1)(2 i)}^{2} x_{(2 i) n}^{2}}\right)^{\frac{\Delta_{2 i}}{2}}\left(\frac{x_{(2 i) n}^{2}}{x_{(2 i-1)(2 i)}^{2} x_{(2 i-1) n}^{2}}\right)^{\frac{\Delta_{2 i-1}}{2}}\right),
\end{aligned}
$$

and the $(r s)$ index in the final product in (3.39) runs over the index set

$$
\mathcal{V}_{\mathrm{OPE}}:=\{(a b) \mid 1 \leq a<b \leq n-1\} \backslash \mathcal{S}_{\mathrm{OPE}},
$$

where we have defined ${ }^{10}$

$$
\begin{aligned}
\mathcal{S}_{\mathrm{OPE}}:= & \left\{((2 j+1)(2 j+2)) \mid 0 \leq j \leq \frac{n}{2}-2\right\} \bigcup\left\{(2(2 j)) \mid 2 \leq j \leq \frac{n}{2}-1\right\} \\
& \bigcup\{((n-2)(n-1))\} .
\end{aligned}
$$

It can be easily checked that the subscripts (rs) take $\left|\mathcal{V}_{\mathrm{OPE}}\right|=\left(\begin{array}{c}n-2 \\ 2\end{array}\right)$ pairs of values. The index set $\mathcal{V}_{\mathrm{OPE}}$ in turn allows us to compute the post-Mellin parameters for the external conformal dimensions via (2.18) (it may be helpful here to consult figure 7 from section 4.3 for a visual representation of the set $\mathcal{V}_{\mathrm{OPE}}$ ):

$$
\begin{aligned}
& \ell_{1}=\sum_{s=3}^{n-1} j_{1 s} \quad \ell_{2}=\sum_{i=1}^{\frac{n}{2}-1} j_{2(2 i+1)} \quad \ell_{n}=0 \\
& \ell_{n-1}=\sum_{r=1}^{n-3} j_{r(n-1)} \quad \ell_{n-2}=j_{1(n-2)}+\sum_{r=3}^{n-4} j_{r(n-2)} \quad \ell_{n-3}=j_{(n-3)(n-1)}+\sum_{r=1}^{n-4} j_{r(n-3)} \\
& \ell_{2 i+2}=j_{1(2 i+2)}+\sum_{s=2 i+3}^{n-1} j_{(2 i+2) s}+\sum_{r=3}^{2 i} j_{r(2 i+2)} \quad\left(1 \leq i \leq \frac{n}{2}-3\right) \\
& \ell_{2 i+1}=\sum_{s=2 i+3}^{n-1} j_{(2 i+1) s}+\sum_{r=1}^{2 i} j_{r(2 i+1)} \quad\left(1 \leq i \leq \frac{n}{2}-3\right) .
\end{aligned}
$$

Now we can use (2.20) and (2.21) to determine the post-Mellin parameters for internal conformal dimensions iteratively. First, focusing on vertices with two incident external legs, we can determine the post-Mellin parameters from previously determined data, as

\footnotetext{
${ }^{10}$ Explicitly, $\mathcal{S}_{\mathrm{OPE}}=\{(12),(34), \ldots,((n-3)(n-2)),(24),(26), \ldots,(2(n-2)),((n-2)(n-1))\}$.
} 
shown:

$$
\bigvee_{\Delta_{\delta_{1}}}^{\Delta_{1}}: \ell_{\delta_{1}} \stackrel{2 \mathcal{J}}{=} \ell_{1}+\ell_{2}=\sum_{s=3}^{n-1} j_{1 s}+\sum_{i=1}^{\frac{n}{2}-1} j_{2(2 i+1)}
$$

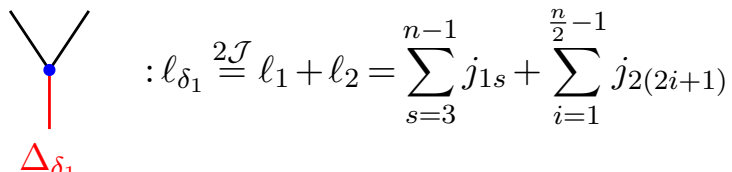

$\Delta_{n-1} \Delta_{n}$

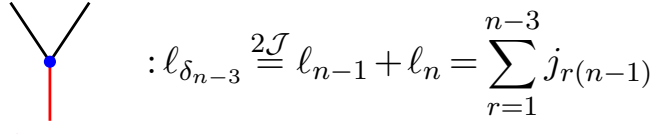

$\Delta_{\delta_{n-3}}$

$$
\bigvee_{\Delta_{\delta_{n-4}}}^{\Delta_{n-3} \Delta_{n-2}}: \ell_{\delta_{n-4}} \stackrel{2 \mathcal{J}}{=} \ell_{n-3}+\ell_{n-2}=j_{(n-3)(n-1)}+\sum_{r=1}^{n-4} j_{r(n-3)}+j_{1(n-2)}+\sum_{r=3}^{n-4} j_{r(n-2)}
$$

$\Delta_{2 i+1} \Delta_{2 i+2}$

V $\ell_{\delta_{2 i}} \stackrel{2 \mathcal{J}}{=} \ell_{2 i+1}+\ell_{2 i+2}=\sum_{s=2 i+3}^{n-1} j_{(2 i+1) s}+\sum_{r=1}^{2 i} j_{r(2 i+1)}+j_{1(2 i+2)}+\sum_{s=2 i+3}^{n-1} j_{(2 i+2) s}$

$\Delta_{\delta_{2 i}}$

$$
+\sum_{r=3}^{2 i} j_{r(2 i+2)}
$$

for $1 \leq i \leq \frac{n}{2}-3$. In fact, in all $\stackrel{2 \mathcal{J}}{=}$ equalities in (3.44), we can freely drop the $2 \mathcal{J}$ as the post-Mellin parameters on the r.h.s. do not share common Mellin parameters.

The post-Mellin parameters for the remaining internal conformal dimensions satisfy

$$
\ell_{\delta_{2 i+1}} \stackrel{2 \mathcal{J}}{=} \ell_{\delta_{2 i-1}}+\ell_{\delta_{2 i}}
$$

for $1 \leq i \leq \frac{n}{2}-3$, thus they need to be determined iteratively. Working out a few explicit cases such as $\ell_{\delta_{3}}, \ell_{\delta_{5}}$ and $\ell_{\delta_{7}}$ allows us to conjecture, and subsequently prove by induction in appendix B.1, the general form

$$
\ell_{\delta_{2 i+1}}=\sum_{s=2 i+3}^{n-1} j_{1 s}+\sum_{r=3}^{2 i+2} \sum_{s=2 i+3}^{n-1} j_{r s}+\sum_{z=i+1}^{\frac{n}{2}-1} j_{2(2 z+1)}
$$

for $1 \leq i \leq \frac{n}{2}-3$.

We now have all the ingredients to write down the full conformal block in the OPE channel. The $(n-3)$ edge factors are given by (2.4) with the post-Mellin parameters as 
determined above. The $(n-2)$ internal vertices of the binary graph, enumerated as follows

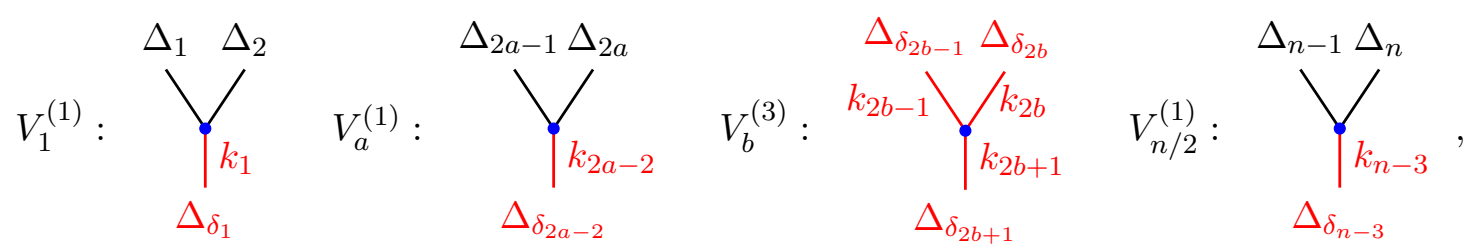

for $2 \leq a \leq n / 2-1$ and $1 \leq b \leq n / 2-2$, correspond to the vertex factors (2.6). Explicitly ${ }^{11}$

$$
\begin{aligned}
& V_{1}^{(1)}=\left(\Delta_{12, \delta_{1}}\right)_{-k_{1}}\left(\Delta_{\delta_{1} 1,2}\right)_{k_{1}+\sum_{s=3}^{n-1} j_{1 s}}\left(\Delta_{\delta_{1} 2,1}\right)_{k_{1}+\sum_{i=1}^{\frac{n}{2}-1} j_{2(2 i+1)}} \\
& \times F_{A}^{(1)}\left[\Delta_{12 \delta_{1}},-h ;\left\{-k_{1}\right\} ;\left\{\Delta_{\delta_{1}}-h+1\right\} ; 1\right] \\
& V_{a}^{(1)}:=\left(\Delta_{(2 a-1)(2 a), \delta_{2 a-2}}\right)_{-k_{2 a-2}}\left(\Delta_{(2 a-1) \delta_{2 a-2}, 2 a}\right)_{k_{2 a-2}+\sum_{s=2 a+1}^{n-1} j_{(2 a-1) s}+\sum_{r=1}^{2 a-2} j_{r(2 a-1)}} \\
& \times\left(\Delta_{(2 a) \delta_{2 a-2}, 2 a-1}\right)_{k_{2 a-2}+j_{1(2 a)}+\sum_{s=2 a+1}^{n-1} j_{(2 a) s}+\sum_{r=3}^{2 a-2} j_{r(2 a)}} \\
& \times F_{A}^{(1)}\left[\Delta_{(2 a-1)(2 a) \delta_{2 a-2},}-h ;\left\{-k_{2 a-2}\right\} ;\left\{\Delta_{\delta_{2 a-2}}-h+1\right\} ; 1\right] \\
& V_{b}^{(3)}:=\left(\Delta_{\delta_{2 b-1} \delta_{2 b}, \delta_{2 b+1}}\right)_{k_{(2 b-1)(2 b),(2 b+1)}+\sum_{s=2 b+1}^{2 b+2} j_{1 s}+\sum_{r=2}^{2 b} j_{r(2 b+1)}+\sum_{r=3}^{2 b} j_{r(2 b+2)}} \\
& \times\left(\Delta_{\delta_{2 b+1} \delta_{2 b-1}, \delta_{2 b}}\right)_{k_{(2 b+1)(2 b-1),(2 b)}+\sum_{s=2 b+3}^{n-1} j_{1 s}+\sum_{r=3}^{2 b} \sum_{s=2 b+3}^{n-1} j_{r s}+\sum_{z=b+1}^{\frac{n}{2}-1} j_{2(2 z+1)}} \\
& \times\left(\Delta_{\delta_{2 b+1} \delta_{2 b}, \delta_{2 b-1}}\right)_{k_{(2 b+1)(2 b),(2 b-1)}}+\sum_{r=2 b+1}^{2 b+2} \sum_{s=2 b+3}^{n-1} j_{r s} \\
& \times F_{A}^{(3)}\left[\Delta_{\delta_{2 b-1} \delta_{2 b} \delta_{2 b+1}},-h ;\left\{-k_{2 b-1},-k_{2 b},-k_{2 b+1}\right\} ;\right. \\
& \left.\left\{\Delta_{\delta_{2 b-1}}-h+1, \Delta_{\delta_{2 b}}-h+1, \Delta_{\delta_{2 b+1}}-h+1\right\} ; 1,1,1\right] \\
& V_{n-2}^{(1)}=\left(\Delta_{(n-1) n, \delta_{n-3}}\right)_{-k_{n-3}}\left(\Delta_{\delta_{n-3}(n-1), n}\right)_{k_{n-3}+\sum_{r=1}^{n-3} j_{r(n-1)}}\left(\Delta_{\delta_{n-3} n, n-1}\right)_{k_{n-3}} \\
& \times F_{A}^{(1)}\left[\Delta_{(n-1) n \delta_{n-3}},-h ;\left\{-k_{n-3}\right\} ;\left\{\Delta_{\delta_{n-3}}-h+1\right\} ; 1\right] .
\end{aligned}
$$

where to work out the linear combination of post-Mellin parameters in $V_{a}^{(1)}$ we used

$$
\frac{1}{2} \ell_{(2 a-1)(2 a), \delta_{2 a-2}}=0 \quad \frac{1}{2} \ell_{(2 a-1) \delta_{2 a-2}, 2 a}=\ell_{2 a-1} \quad \frac{1}{2} \ell_{(2 a) \delta_{2 a-2}, 2 a-1}=\ell_{2 a},
$$

for $2 \leq a \leq n / 2-1$, and in $V_{b}^{(3)}$ we used

$$
\begin{aligned}
\frac{1}{2} \ell_{\delta_{2 b-1} \delta_{2 b}, \delta_{2 b+1}} & =\sum_{s=2 b+1}^{2 b+2} j_{1 s}+\sum_{r=2}^{2 b} j_{r(2 b+1)}+\sum_{r=3}^{2 b} j_{r(2 b+2)} \\
\frac{1}{2} \ell_{\delta_{2 b+1} \delta_{2 b-1}, \delta_{2 b}} & =\sum_{s=2 b+3}^{n-1} j_{1 s}+\sum_{r=3}^{2 b} \sum_{s=2 b+3}^{n-1} j_{r s}+\sum_{z=b+1}^{\frac{n}{2}-1} j_{2(2 z+1)} \\
\frac{1}{2} \ell_{\delta_{2 b+1} \delta_{2 b}, \delta_{2 b-1}} & =\sum_{r=2 b+1}^{2 b+2} \sum_{s=2 b+3}^{n-1} j_{r s},
\end{aligned}
$$

\footnotetext{
${ }^{11}$ To write the vertex factors compactly, we made the additional stipulation that an ill-defined post-Mellin parameter, $j_{(n-2)(n-1)}$, which appears in the subscript of one of the Pochhammer symbols of $V_{n / 2-1}^{(1)}$ as a consequence of our compact rewriting, should be set to zero.
} 
for $1 \leq b \leq n / 2-2$, which can both be verified easily. Substituting all the edge and vertex factors above into (2.2)-(2.3) gives the $n$-point OPE channel block.

In ref. [61], a power-series expansion was worked out for the special case of the $n=6$ block. ${ }^{12}$ Notably, the same generalized hypergeometric function makes an appearance in both their paper and the result above. For $n=6$, it can be seen from (3.48) that in all exactly one factor of the Lauricella function $F_{A}^{(3)}$ appears in the vertex factors, which is directly related to the Kampé de Fériet function via (A.4). Precisely the same Kampé de Fériet function appeared in the result of ref. [61]. The choice of cross-ratios in that paper differs from the general choice made above in (3.35), so to make a precise comparison, we can start with the cross-ratios of ref. [61] and apply to them the Feynman rules of section 2. We confirmed that doing so exactly reproduces the conformal block of ref. [61].

This section generalizes this result to any even $n \geq 6 .{ }^{13}$ At higher $n$, precisely $(n / 2-2)$ factors of the Lauricella function of three variables $F_{A}^{(3)}$ (equivalently $(n / 2-2)$ factors of the Kampé de Fériet function) will appear in the power series expansion. ${ }^{14}$

\section{From Mellin amplitudes to conformal blocks}

In this section we will prove the Feynman prescription for conformal blocks for the examples considered in the previous section. As outlined in section 1, our starting point will be certain canonical tree-level Witten diagrams in an effective $\phi^{3}$ scalar field theory in AdS. We will write down their Mellin amplitudes using the Feynman rules for Mellin amplitude [68-70], from which we will be able to extract the desired conformal blocks via single-trace projections. A canonical Witten diagram is a tree-level Witten diagram of the same topology as the conformal block we are interested in computing, with generic scalar dimensions running along each edge. Since we will only be interested in the contribution coming from singletrace exchanges, and not the full amplitude, we would like to project onto the single-trace part of this Witten diagram. This is convenient to do in large $N$ theories, since the poles of the meromorphic Mellin amplitude correspond precisely to the exchange of single-trace primaries. Evaluating the residue at these poles furnishes the required projection.

Concretely, the Mellin amplitude $\mathcal{M}_{n}\left(\gamma_{i j}\right)$ for an $n$-point Witten diagram, whose position space ampitude is denoted $A_{n}$, is defined via a multi-dimensional inverse Mellin transform,

$$
A_{n}=\mathcal{N}\left(\prod_{(r s) \in \mathcal{U} \bigcup \mathcal{V}} \int \frac{d \gamma_{r s}}{2 \pi i}\right) \mathcal{M}_{n}\left(\gamma_{i j}\right)\left(\prod_{(i j) \in \mathcal{U} \bigcup \mathcal{V} \bigcup \mathcal{D}} \frac{\Gamma\left(\gamma_{i j}\right)}{\left(x_{i j}^{2}\right)^{\gamma_{i j}}}\right)
$$

where $A_{n}$ is an AdS integral over products of bulk-to-bulk and bulk-to-boundary propaga-

\footnotetext{
${ }^{12}$ The authors of ref. [61] referred to it as the "snowflake channel," which is the same as the "OPE channel" above.

${ }^{13}$ Recall that the "OPE channel" in this paper is well-defined only for even number of external operators.

${ }^{14}$ The remaining vertex factors in (3.48) contribute one factor of the Lauricella function $F_{A}^{(1)}$ each, but this function can be trivially expressed in terms of Gamma functions or Pochhammer symbols; see (A.2).
} 
tors which are normalized as follows: in Poincaré coordinates $z=\left(z_{0}, z^{i}\right) \in \mathbb{R}^{+} \times \mathbb{R}^{d}$,

$$
\begin{aligned}
\hat{G}_{\Delta}(w, z) & =\left(\frac{\xi(w, z)}{2}\right)^{\Delta}{ }_{2} F_{1}\left[\frac{\Delta}{2}, \frac{\Delta+1}{2} ; \Delta-\frac{d}{2}+1 ; \xi(w, z)^{2}\right] \\
\xi(w, z) & =\frac{2 w_{0} z_{0}}{w_{0}^{2}+z_{0}^{2}+\left(w^{i}-z^{i}\right)^{2}} \\
\hat{K}_{\Delta}\left(x^{i}, z\right) & =\frac{z_{0}^{\Delta}}{\left(z_{0}^{2}+\left(z^{i}-x^{i}\right)^{2}\right)^{\Delta}} .
\end{aligned}
$$

The contours of integration on the r.h.s. of (4.1) run parallel to the imaginary axis for $\operatorname{Re} \gamma_{r s}>0$ such that they separate out the semi-infinite sequence of poles running to the left or to the right. The overall normalization constant $\mathcal{N}$ will be fixed shortly. The set $\mathcal{U} \cup \mathcal{V}$ is the index set of $n(n-3) / 2$ independent Mellin variables $\gamma_{i j}$ and the set $\mathcal{D}$ is the index set of the $n$ dependent Mellin variables. We have chosen to decompose the independent variable index set into a union of two disjoint subsets $\mathcal{U}$ and $\mathcal{V}$. The precise prescription for this choice of sets was explained in section 2.1 and illustrated in section 3. Briefly, this choice will be dictated by the choice of cross-ratios and the channel (i.e. binary tree topology) for the precise conformal block we wish to extract from $A_{n}$. To stress this dependence, we will call the sets $\mathcal{U}_{\text {chan }}, \mathcal{V}_{\text {chan }}$ and $\mathcal{D}_{\text {chan }}$. The choice of cross-ratios and the index sets served as the input in section 3 for writing down the conformal block using the proposed Feynman rules. In this section, this choice will serve as the input for deriving the block from Mellin amplitudes.

The union of all three sets $\mathcal{U}_{\text {chan }}, \mathcal{V}_{\text {chan }}$ and $\mathcal{D}_{\text {chan }}$ gives the full range of indices (2.16) associated with the product over Gamma functions and powers of pairwise distances in (4.1). This product over powers of pairwise distances was called the "Mellin product"; see (2.13). For a canonical choice of index sets, the Mellin product admits a convenient rewriting, to be substituted in (4.1), in terms of the chosen cross-ratios as shown in (2.15) and repeated below:

$$
\left(\prod_{(i j) \in \mathcal{U}_{\text {chan }}} \bigcup \mathcal{V}_{\text {chan }} \bigcup \mathcal{D}_{\text {chan }} \frac{1}{\left(x_{i j}^{2}\right)^{\gamma_{i j}}}\right)=W_{n, \text { chan }}^{0}\left(x_{i}\right)\left(\prod_{i=1}^{n-3} u_{i}^{s_{i} / 2}\right)\left(\prod_{(r s) \in \mathcal{V}_{\text {chan }}} v_{r s}^{-\gamma_{r s}}\right)
$$

where $s_{i}$ are the Mandelstam invariants associated with the internal legs (and expressible in terms of Mellin variables drawn from the index sets $\mathcal{U}_{\text {chan }}$ and $\left.\mathcal{V}_{\text {chan }}\right)$, while $W_{n \text {,chan }}^{0}\left(x_{i}\right)$ is the leg-factor which depends solely on external conformal dimensions and position coordinates. We note that we have indexed the Mandelstam invariants and the cross-ratios in a manner that allows us to write (4.3) as displayed.

To evaluate the single-trace contribution to $A_{n}$, one needs the Mellin amplitude $\mathcal{M}\left(\gamma_{i j}\right)$ for the Witten diagram. For tree-level scalar Witten diagrams, the Mellin amplitude is readily available via the "Feynman rules for Mellin amplitudes" [68-70]. According to these rules, in the normalization conventions we are following, the Mellin amplitude of a scalar $n$-point tree-level Witten diagram in a $\phi^{3}$ theory is constructed as follows:

- Label the internal lines of the Witten diagram with an index $i$ running from 1 to $n-3$, and to it associate an integer parameter $k_{i}$ (which will double as single-trace 
parameters) and a factor of

$$
E_{i}^{\text {Mellin }}:=\frac{1}{k_{i} !} \frac{\left(\Delta_{\delta_{i}}-h+1\right)_{k_{i}}}{\frac{\Delta_{\delta_{i}}-s_{i}}{2}+k_{i}} \quad(1 \leq i \leq n-3),
$$

where $s_{i}$ is the Mandelstam invariant associated to that leg, and $\Delta_{\delta_{i}}$ is the conformal dimension of the dual operator running along the line.

- Label each internal vertex of the diagram with an index $j$ running from 1 to $n-2$ and assign a factor of

$$
\begin{aligned}
V_{j}^{\text {Mellin }} & :=\frac{1}{2} \Gamma\left(\Delta_{a b c},-h\right) \\
& \times F_{A}^{(3)}\left[\Delta_{a b c},-h ;\left\{-k_{a},-k_{b},-k_{c}\right\} ;\left\{\Delta_{a}-h+1, \Delta_{b}-h+1, \Delta_{c}-h+1\right\} ; 1,1,1\right],
\end{aligned}
$$

where $\Delta_{a}, \Delta_{b}$ and $\Delta_{c}$ are the conformal dimensions incident at the vertex, and $k_{a}, k_{b}$ and $k_{c}$ are the respective integer parameters (or single-trace parameters) associated with the internal exchanged dimensions. Set the integer parameter to zero if the conformal dimension associated to it is an external dimension.

Then for the choice of normalization constant,

$$
\mathcal{N}:=\pi^{(n-2) h}\left(\prod_{i=1}^{n-3} \frac{1}{\Gamma\left(\Delta_{\delta_{i}}\right)}\right)\left(\prod_{i=1}^{n} \frac{1}{\Gamma\left(\Delta_{i}\right)}\right),
$$

where $\Delta_{i}$ are the external conformal dimensions and $\Delta_{\delta_{i}}$ are the internal exchanged dimensions, the Mellin amplitude is given by

$$
\mathcal{M}_{n}\left(\gamma_{i j}\right)=\left(\prod_{i=1}^{n-3} \sum_{k_{i}=0}^{\infty}\right)\left(\prod_{i=1}^{n-3} E_{i}^{\mathrm{Mellin}}\right)\left(\prod_{i=1}^{n-2} V_{i}^{\mathrm{Mellin}}\right) .
$$

With the Mellin amplitude in hand, we can proceed to evaluate the single-trace projection of the position space amplitude $A_{n}$, by performing the contour integrals in (4.1). The single-trace contribution comes from the poles of the Mellin amplitude. From the point of view of the Feynman prescription for Mellin amplitudes, these arise from simple poles occurring in the denominator of the Mellin amplitude edge factors (4.4). In terms of Mandelstam invariants, these poles occur at

$$
s_{i}=\Delta_{\delta_{i}}+2 k_{i} \quad(1 \leq i \leq n-3)
$$

which correspond to putting the internal legs on-shell in the auxiliary momentum space. These poles should be viewed as lying in the complex $\gamma_{r s}$ planes for $(r s) \in \mathcal{U}_{\text {chan }}$, and our task is to evaluate the residue at these poles.

Before we do so, we point out that precisely the same Lauricella functions as those in (4.5) appeared in (2.6) in the Feynman rules for conformal blocks. This is expected for the simple reason that the vertex factors (4.5) are independent of Mellin variables $\gamma_{i j}$, so they remain unaffected through the following computation of Mellin integrals. 
Turning to evaluating the residue at the "single-trace poles" (4.8) in the " $\mathcal{U}_{\text {chan }}$-plane," we obtain the following single-trace projection of the AdS diagram, denoted $A_{n}^{\text {s.t. }}$,

$$
\begin{aligned}
& A_{n}^{\text {s.t. }}:=\mathcal{N} W_{n, \text { chan }}^{0}\left(x_{i}\right)\left(\prod_{i=1}^{n-3} \sum_{k_{i}=0}^{\infty}\right)\left(\prod_{i=1}^{n-3} \frac{u_{i}^{\frac{\Delta_{\delta_{i}}}{2}+k_{i}}}{k_{i} !}\left(\Delta_{\delta_{i}}-h+1\right)_{k_{i}}\right)\left(\prod_{i=1}^{n-2} V_{i}^{\mathrm{Mellin}}\right) \\
& \times\left(\prod_{(r s) \in \mathcal{V}_{\text {chan }}} \int \frac{d \gamma_{r s}}{2 \pi i}\right)\left(\prod_{(i j) \in \mathcal{V}_{\text {chan }}} \Gamma\left(\gamma_{i j}\right)\right)\left(\left.\prod_{(i j) \in \mathcal{U}_{\text {chan }}} \bigcup \mathcal{D}_{\text {chan }} \Gamma\left(\gamma_{i j}\right)\right|_{\text {s.t. }}\right)\left(\prod_{(r s) \in \mathcal{V}_{\text {chan }}} v_{r s}^{-\gamma_{r s}}\right),
\end{aligned}
$$

where $\left.\Gamma\left(\gamma_{i j}\right)\right|_{\text {s.t. }}$ stands for Gamma functions with arguments from the index sets $\mathcal{U}_{\text {chan }}$ and $\mathcal{D}_{\text {chan }}$ evaluated at the poles (4.8). ${ }^{15}$ Equation (4.9) is proportional to the desired conformal block. More precisely,

$$
\begin{aligned}
& W_{n, \text { chan }}\left(x_{i}\right)=\frac{\mathcal{N} W_{n, \text { chan }}^{0}\left(x_{i}\right)}{\left(\prod_{i=1}^{n-2} f_{i}\right)}\left(\prod_{i=1}^{n-3} \sum_{k_{i}=0}^{\infty}\right)\left(\prod_{i=1}^{n-3} \frac{u_{i}^{\frac{\Delta_{\delta_{i}}}{2}+k_{i}}}{k_{i} !}\left(\Delta_{\delta_{i}}-h+1\right)_{k_{i}}\right)\left(\prod_{i=1}^{n-2} V_{i}^{\mathrm{Mellin}}\right) \\
& \times\left(\prod_{(r s) \in \mathcal{V}_{\text {chan }}} \int \frac{d \gamma_{r s}}{2 \pi i}\right)\left(\prod_{(i j) \in \mathcal{V}_{\text {chan }}} \Gamma\left(\gamma_{i j}\right)\right)\left(\left.\prod_{(i j) \in \mathcal{U}_{\text {chan }} \bigcup \mathcal{D}_{\text {chan }}} \Gamma\left(\gamma_{i j}\right)\right|_{\text {s.t. }}\right)\left(\prod_{(r s) \in \mathcal{V}_{\text {chan }}} v_{r s}^{-\gamma_{r s}}\right)
\end{aligned}
$$

furnishes a mixed series-integral representation of the desired conformal block, where $f_{i}=$ $C_{\Delta_{a_{i}} \Delta_{b_{i}} \Delta_{c_{i}}}$ are the known $(n-2)$ MFT OPE coefficients, one for each internal vertex of the binary unrooted tree representing the AdS diagram with scalars of conformal dimensions $\Delta_{a_{i}}, \Delta_{b_{i}}$ and $\Delta_{c_{i}}$ on the incident edges.

To obtain an integral-free representation of the block, one must evaluate the residual $\left(\begin{array}{c}n-2 \\ 2\end{array}\right)$-dimensional contour integrals in the second line of (4.10). This will introduce $\left(\begin{array}{c}n-2 \\ 2\end{array}\right)$ new summations. Evaluating these integrals in general for an arbitrary $n$-point conformal block in an arbitrary channel is not clear to us. Instead, in the remainder of this section, we will focus on evaluating these integrals explicitly for the three classes of examples from section 3. We will reproduce the blocks as prescribed by the Feynman rules of section 2, which serves as a highly non-trivial check of the proposed Feynman rules.

Before specializing to specific examples, we can do further general manipulations. First, isolating the integral in the second line of (4.10),

$I:=\left(\prod_{(r s) \in \mathcal{V}_{\text {chan }}} \int \frac{d \gamma_{r s}}{2 \pi i}\right)\left(\prod_{(r s) \in \mathcal{V}_{\text {chan }}} \Gamma\left(\gamma_{r s}\right)\right)\left(\left.\prod_{(i j) \in \mathcal{U}_{\text {chan }} \bigcup \mathcal{D}_{\text {chan }}} \Gamma\left(\gamma_{i j}\right)\right|_{\text {s.t. }}\right)\left(\prod_{(r s) \in \mathcal{V}_{\text {chan }}} v_{r s}^{-\gamma_{r s}}\right)$,

\footnotetext{
${ }^{15}$ We note that the dependent Mellin variables $\gamma_{i j}$ with $(i j) \in \mathcal{D}_{\text {chan }}$ in the Gamma functions are assumed to have already been expressed in terms of the independent Mellin variables from the sets $\mathcal{U}_{\text {chan }}$ and $\mathcal{V}_{\text {chan }}$ Still, for brevity, we prefer to use the notation in the second line of (4.9). We also emphasize the obvious fact that the Gamma functions with arguments from the index set $\mathcal{V}_{\text {chan }}$ remain unaffected after taking the single-trace residues.
} 
we rewrite each factor of $v_{r s}^{-\gamma_{r s}}$ above by introducing an additional contour integral, as follows. ${ }^{16}$

$$
v_{r s}^{-\gamma_{r s}}=\frac{1}{\Gamma\left(\gamma_{r s}\right)} \int \frac{d \widetilde{\gamma}_{r s}}{2 \pi i} \Gamma\left(-\widetilde{\gamma}_{r s}\right) \Gamma\left(\widetilde{\gamma}_{r s}+\gamma_{r s}\right)\left(v_{r s}-1\right)^{\widetilde{\gamma}_{r s}},
$$

where the $\widetilde{\gamma}_{r s}$ contour runs vertically such that it separates the semi-infinite sequence of poles running to the left and to right of origin. Then we get

$$
I=\left(\prod_{(r s) \in \mathcal{V}_{\text {chan }}} \int \frac{d \widetilde{\gamma}_{r s}}{2 \pi i} \int \frac{d \gamma_{r s}}{2 \pi i} \Gamma\left(-\widetilde{\gamma}_{r s}\right) \Gamma\left(\widetilde{\gamma}_{r s}+\gamma_{r s}\right)\left(v_{r s}-1\right)^{\widetilde{\gamma}_{r s}}\right)\left(\left.\prod_{(i j) \in \mathcal{D}_{\mathrm{OPE}} \bigcup \mathcal{U}_{\mathrm{OPE}}} \Gamma\left(\gamma_{i j}\right)\right|_{\text {s.t. }}\right),
$$

where we switched the order of integrals. The rewriting in (4.13) makes it easier to obtain a convergent series expansion of the conformal block in powers of $\left(1-v_{r s}\right)$ as desired (see (2.2)-(2.3)). The overall strategy now will be to repeatedly use the first Barnes lemma [74],

$$
\int_{-i \infty}^{+i \infty} \frac{d s}{2 \pi i} \Gamma\left(a_{1}+s\right) \Gamma\left(a_{2}+s\right) \Gamma\left(b_{1}-s\right) \Gamma\left(b_{2}-s\right)=\frac{\Gamma\left(a_{1}+b_{1}\right) \Gamma\left(a_{1}+b_{2}\right) \Gamma\left(a_{2}+b_{1}\right) \Gamma\left(a_{2}+b_{2}\right)}{\Gamma\left(a_{1}+a_{2}+b_{1}+b_{2}\right)},
$$

to evaluate all $\gamma_{r s}$ integrals, which it turns out will leave us with trivial-to-evaluate $\widetilde{\gamma}_{r s}$ contour integrals.

\subsection{Seven-point mixed channel}

To obtain the 7-point "mixed-channel" conformal block (topology shown in figure 1b), we start with the following "canonical" tree-level AdS diagram:

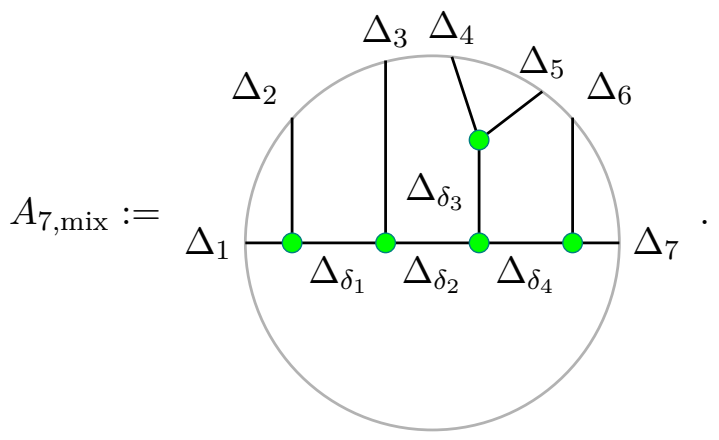

Here we have labeled the external scalar operators with their conformal dimensions but suppressed the insertion coordinates (e.g. $\Delta_{1}$ should be understood as a scalar operator of conformal dimension $\Delta_{1}$ inserted at boundary coordinate $x_{1}$ ). By design, the operator insertions and exchanged operator labels in the canonical Witten diagram above match with

${ }^{16}$ Use the Mellin-Barnes representation,

$$
\frac{1}{(x+y)^{a}}=\frac{1}{\Gamma(a)} \int \frac{d s}{2 \pi i} \Gamma(-s) \Gamma(s+a) x^{s} y^{-a-s}
$$

with $x=v_{r s}-1, y=1$ and $a=\gamma_{r s}$. The contour of integration is chosen such that it separates the poles of $\Gamma(s+a)$ from those of $\Gamma(-s)$; see e.g. the discussion around [53, eq. (B.11)]. 


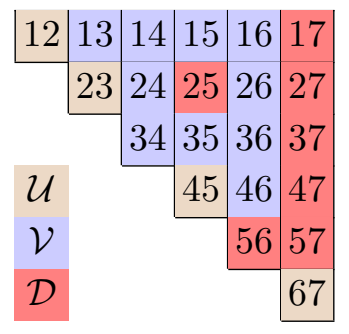

Figure 5. Color-coded matrix displaying the choice of Mellin variable index subsets $\mathcal{U}_{7 \text {,mix }}, \mathcal{V}_{7 \text {, mix }}$ and $\mathcal{D}_{7, \text { mix }}$.

the corresponding binary graph of the 7-point conformal block shown in figure 1b. Finally, the green disks represent cubic interaction vertices to be integrated over all of $\mathrm{AdS}_{d+1}$.

As previously noted, to express the conformal block, one must start by making a choice of independent conformal cross-ratios. To reproduce the block obtained via the Feynman rules of section 2, we will utilize the same choice of cross-ratios as in section 3.1, which comes with associated index sets for the dependent and (a subset of) independent Mellin variables, $\mathcal{D}_{7, \text { mix }}$ and $\mathcal{V}_{7, \text { mix }}$ respectively (see (3.3) and (3.7)). Using (2.16) we can determine the remaining independent Mellin variables, associated with the index set $\mathcal{U}_{7 \text {,mix }}$,

$$
\mathcal{U}_{7, \text { mix }}=\{(12),(23),(45),(67)\} .
$$

With the help of a color-coded upper-triangular matrix, we can present these sets visually as shown in figure 5 . With these choices in place, the Mellin product admits a rewriting in terms of the cross-ratios as shown in (3.5), which we substitute into (4.1).

The other ingredient which goes into (4.1) is the Mellin amplitude $\mathcal{M}_{7}\left(\gamma_{i j}\right)$ for the seven-point AdS diagram above. According to the Feynman rules for Mellin amplitudes, the Mellin amplitude in this case is given by (4.7) for $n=7$ with the edge factors given in (4.4) and the vertex factors taking the explicit form:

$$
\begin{aligned}
V_{1}^{\text {Mellin }}= & \frac{1}{2} \Gamma\left(\Delta_{12 \delta_{1}},-h\right) F_{A}^{(1)}\left[\Delta_{12 \delta_{1},}-h ;\left\{-k_{1}\right\} ;\left\{\Delta_{\delta_{1}}-h+1\right\} ; 1\right] \\
V_{2}^{\text {Mellin }}= & \frac{1}{2} \Gamma\left(\Delta_{3 \delta_{1} \delta_{2}},-h\right) F_{A}^{(2)}\left[\Delta_{3 \delta_{1} \delta_{2}},-h ;\left\{-k_{1},-k_{2}\right\} ;\left\{\Delta_{\delta_{1}}-h+1, \Delta_{\delta_{2}}-h+1\right\} ; 1,1\right] \\
V_{3}^{\text {Mellin }}= & \frac{1}{2} \Gamma\left(\Delta_{\delta_{2} \delta_{3} \delta_{4}},-h\right) \\
& \times F_{A}^{(3)}\left[\Delta_{\delta_{2} \delta_{3} \delta_{4}}-h ;\left\{-k_{2},-k_{3},-k_{4}\right\} ;\left\{\Delta_{\delta_{2}}-h+1, \Delta_{\delta_{3}}-h+1, \Delta_{\delta_{4}}-h+1\right\} ; 1,1,1\right] \\
V_{4}^{\text {Mellin }}= & \frac{1}{2} \Gamma\left(\Delta_{45 \delta_{3}},-h\right) F_{A}^{(1)}\left[\Delta_{45 \delta_{3}},-h ;\left\{-k_{3}\right\} ;\left\{\Delta_{\delta_{3}}-h+1\right\} ; 1\right] \\
V_{5}^{\text {Mellin }}= & \frac{1}{2} \Gamma\left(\Delta_{67 \delta_{4}},-h\right) F_{A}^{(1)}\left[\Delta_{67 \delta_{4}},-h ;\left\{-k_{4}\right\} ;\left\{\Delta_{\delta_{4}}-h+1\right\} ; 1\right] .
\end{aligned}
$$

The single-trace projection of the Witten diagram (4.16), described in general terms in the discussion preceding this example, then leads to (4.9) which is proportional to the desired conformal block. This projection involves evaluating the residue at the "single-trace 
poles," occuring at

$$
\gamma_{12}=\Delta_{12, \delta_{1}}-k_{1} \quad \gamma_{23}=\Delta_{3 \delta_{1}, \delta_{2}}+k_{1,2}-\gamma_{13} \quad \gamma_{45}=\Delta_{45, \delta_{3}}-k_{3} \quad \gamma_{67}=\Delta_{67, \delta_{4}}-k_{4} .
$$

The block itself is given by (4.10) by projecting out the theory dependent OPE coefficients:

$$
f_{1}=C_{\Delta_{1} \Delta_{2} \Delta_{\delta_{1}}} \quad f_{2}=C_{\Delta_{3} \Delta_{\delta_{1}} \Delta_{\delta_{2}}} \quad f_{3}=C_{\Delta_{\delta_{2}} \Delta_{\delta_{3}} \Delta_{\delta_{4}}} \quad f_{4}=C_{\Delta_{4} \Delta_{5} \Delta_{\delta_{3}}} \quad f_{5}=C_{\Delta_{6} \Delta_{7} \Delta_{\delta_{4}}} .
$$

The MFT OPE coefficients above take the well known form,

$$
C_{\Delta_{a} \Delta_{b} \Delta_{c}}=\frac{\pi^{h}}{2} \Gamma\left(\Delta_{a b c,}-h\right) \frac{\Gamma\left(\Delta_{a b, c}\right) \Gamma\left(\Delta_{b c, a}\right) \Gamma\left(\Delta_{c a, b}\right)}{\Gamma\left(\Delta_{a}\right) \Gamma\left(\Delta_{b}\right) \Gamma\left(\Delta_{c}\right)} .
$$

The precise form of these OPE coefficients will be utilized at the end of the computation.

The non-trivial computation which needs to be done is the contour integral on the second line of (4.10), or equivalently the integral (4.14). The arguments of Gamma functions in the product $\left(\left.\prod_{(i j) \in \mathcal{D}_{7, \text { mix }} \mathcal{U}_{7, \text { mix }}} \Gamma\left(\gamma_{i j}\right)\right|_{\text {s.t. }}\right)$ are expressed entirely in terms of Mellin variables from the index set $\mathcal{V}_{7, \text { mix }}$. Four of them were shown in (4.19) which correspond to the index set $\mathcal{U}_{7, \text { mix }}$; the other seven, corresponding to the index set $\mathcal{D}_{7 \text {,mix }}$ take the form

$$
\begin{aligned}
\gamma_{17} & =\Delta_{1 \delta_{1}, 2}+k_{1}-\gamma_{13}-\gamma_{14}-\gamma_{15}-\gamma_{16} \\
\gamma_{25} & =\Delta_{\delta_{2} \delta_{3}, \delta_{4}}+k_{23,4}-\gamma_{14}-\gamma_{15}-\gamma_{24}-\gamma_{34}-\gamma_{35} \\
\gamma_{27} & =\Delta_{2 \delta_{4}, 13 \delta_{3}}+k_{4,3}+\gamma_{13}+\gamma_{14}+\gamma_{15}-\gamma_{26}+\gamma_{34}+\gamma_{35} \\
\gamma_{37} & =\Delta_{3 \delta_{2}, \delta_{1}}+k_{2,1}-\gamma_{34}-\gamma_{35}-\gamma_{36} \\
\gamma_{47} & =\Delta_{4 \delta_{3}, 5}+k_{3}-\gamma_{14}-\gamma_{24}-\gamma_{34}-\gamma_{46} \\
\gamma_{56} & =\Delta_{6 \delta_{4}, 7}+k_{4}-\gamma_{16}-\gamma_{26}-\gamma_{36}-\gamma_{46} \\
\gamma_{57} & =\Delta_{57,46 \delta_{2}}-k_{2}+\gamma_{14}+\gamma_{16}+\gamma_{24}+\gamma_{26}+\gamma_{34}+\gamma_{36}+\gamma_{46} .
\end{aligned}
$$

These were obtained by substituting (4.19) into (3.4).

Explicitly, $I$ in (4.14) then takes the form

$$
\begin{aligned}
I= & \left(\prod_{(r s) \in \mathcal{V}_{7, \text { mix }}} \int \frac{d \widetilde{\gamma}_{r s}}{2 \pi i} \int \frac{d \gamma_{r s}}{2 \pi i} \Gamma\left(-\widetilde{\gamma}_{r s}\right) \Gamma\left(\widetilde{\gamma}_{r s}+\gamma_{r s}\right)\left(v_{r s}-1\right)^{\gamma_{r s}}\right) \Gamma\left(\Delta_{12, \delta_{1}}-k_{1}\right) \\
& \times \Gamma\left(\Delta_{3 \delta_{1}, \delta_{2}}+k_{1,2}-\gamma_{13}\right) \Gamma\left(\Delta_{45, \delta_{3}}-k_{3}\right) \Gamma\left(\Delta_{67, \delta_{4}}-k_{4}\right) \\
& \times \Gamma\left(\Delta_{1 \delta_{1}, 2}+k_{1}-\gamma_{13}-\gamma_{14}-\gamma_{15}-\gamma_{16}\right) \\
& \times \Gamma\left(\Delta_{\delta_{2} \delta_{3}, \delta_{4}}+k_{23,4}-\gamma_{14}-\gamma_{15}-\gamma_{24}-\gamma_{34}-\gamma_{35}\right) \\
& \times \Gamma\left(\Delta_{2 \delta_{4}, 13 \delta_{3}}+k_{4,3}+\gamma_{13}+\gamma_{14}+\gamma_{15}-\gamma_{26}+\gamma_{34}+\gamma_{35}\right) \\
& \times \Gamma\left(\Delta_{3 \delta_{2}, \delta_{1}}+k_{2,1}-\gamma_{34}-\gamma_{35}-\gamma_{36}\right) \\
& \times \Gamma\left(\Delta_{4 \delta_{3}, 5}+k_{3}-\gamma_{14}-\gamma_{24}-\gamma_{34}-\gamma_{46}\right) \\
& \times \Gamma\left(\Delta_{6 \delta_{4}, 7}+k_{4}-\gamma_{16}-\gamma_{26}-\gamma_{36}-\gamma_{46}\right) \\
& \times \Gamma\left(\Delta_{57,46 \delta_{2}}-k_{2}+\gamma_{14}+\gamma_{16}+\gamma_{24}+\gamma_{26}+\gamma_{34}+\gamma_{36}+\gamma_{46}\right)
\end{aligned}
$$


We first evaluate the 10-dimensional contour integral over the $\gamma_{r s}$ variables for $(r s) \in \mathcal{V}_{7 \text {,mix }}$. We perform the contour integrations one at a time, in the following order:

$\gamma_{16}, \gamma_{36}, \gamma_{26}, \gamma_{46}, \gamma_{35}, \gamma_{15}, \gamma_{24}, \gamma_{34}, \gamma_{13}, \gamma_{14}$

With this choice of ordering, we are able to make direct use of the first Barnes lemma (4.15) at every step. ${ }^{17}$ To keep the manuscript to a reasonable length, we refrain from including the lengthy but straightforward computational details, and merely present the final result of this 10-fold contour integral:

$$
\begin{aligned}
I= & \left(\prod_{(r s) \in \mathcal{V}_{7, \text { mix }}} \int \frac{d \widetilde{\gamma}_{r s}}{2 \pi i} \Gamma\left(-\widetilde{\gamma}_{r s}\right)\left(v_{r s}-1\right)^{\gamma_{r s}}\right) \\
& \times\left(\Gamma\left(\Delta_{12, \delta_{1}}-k_{1}\right) \Gamma\left(\widetilde{\gamma}_{24}+\widetilde{\gamma}_{26}+\Delta_{2 \delta_{1}, 1}+k_{1}\right) \Gamma\left(\widetilde{\gamma}_{13}+\widetilde{\gamma}_{14}+\widetilde{\gamma}_{15}+\widetilde{\gamma}_{16}+\Delta_{1 \delta_{1}, 2}+k_{1}\right)\right) \\
& \times\left(\Gamma\left(\widetilde{\gamma}_{14}+\widetilde{\gamma}_{15}+\widetilde{\gamma}_{16}+\widetilde{\gamma}_{24}+\widetilde{\gamma}_{26}+\Delta_{\delta_{1} \delta_{2}, 3}+k_{12,}\right) \Gamma\left(\widetilde{\gamma}_{13}+\Delta_{3 \delta_{1}, \delta_{2}}+k_{1,2}\right)\right. \\
& \left.\times \Gamma\left(\widetilde{\gamma}_{34}+\widetilde{\gamma}_{35}+\widetilde{\gamma}_{36}+\Delta_{3 \delta_{2}, \delta_{1}}+k_{2,1}\right)\right) \\
& \times\left(\Gamma\left(\widetilde{\gamma}_{14}+\widetilde{\gamma}_{15}+\widetilde{\gamma}_{24}+\widetilde{\gamma}_{34}+\widetilde{\gamma}_{35}+\Delta_{\delta_{2} \delta_{3}, \delta_{4}}+k_{23,4}\right) \Gamma\left(\widetilde{\gamma}_{46}+\Delta_{\delta_{3} \delta_{4}, \delta_{2}}+k_{34,2}\right)\right. \\
& \left.\times \Gamma\left(\widetilde{\gamma}_{16}+\widetilde{\gamma}_{26}+\widetilde{\gamma}_{36}+\Delta_{\delta_{2} \delta_{4}, \delta_{3}}+k_{24,3}\right)\right) \\
& \times\left(\Gamma\left(\Delta_{45, \delta_{3}}-k_{3}\right) \Gamma\left(\widetilde{\gamma}_{14}+\widetilde{\gamma}_{24}+\widetilde{\gamma}_{34}+\widetilde{\gamma}_{46}+\Delta_{4 \delta_{3}, 5}+k_{3}\right) \Gamma\left(\widetilde{\gamma}_{15}+\widetilde{\gamma}_{35}+\Delta_{5 \delta_{3}, 4}+k_{3}\right)\right) \\
& \left.\times \frac{1}{\Gamma\left(\widetilde{\gamma}_{13}+\widetilde{\gamma}_{14}+\widetilde{\gamma}_{15}+\widetilde{\gamma}_{16}+\widetilde{\gamma}_{24}+\widetilde{\gamma}_{26}+\Delta_{\delta_{1}}+2 k_{1}\right)}\left(\Delta_{7 \delta_{4}, 6}+k_{4}\right) \Gamma\left(\widetilde{\gamma}_{16}+\widetilde{\gamma}_{26}+\widetilde{\gamma}_{36}+\widetilde{\gamma}_{46}+\Delta_{6 \delta_{4}, 7}+k_{4}\right)\right) \\
& \times \frac{1}{\Gamma\left(\widetilde{\gamma}_{14}+\widetilde{\gamma}_{15}+\widetilde{\gamma}_{16}+\widetilde{\gamma}_{24}+\widetilde{\gamma}_{26}+\widetilde{\gamma}_{34}+\widetilde{\gamma}_{35}+\widetilde{\gamma}_{36}+\Delta_{\delta_{2}}+2 k_{2}\right)} \\
& \times \frac{1}{\Gamma\left(\widetilde{\gamma}_{14}+\widetilde{\gamma}_{15}+\widetilde{\gamma}_{24}+\widetilde{\gamma}_{34}+\widetilde{\gamma}_{35}+\widetilde{\gamma}_{46}+\Delta_{\delta_{3}}+2 k_{3}\right)} \\
& \times \frac{1}{\Gamma\left(\widetilde{\gamma}_{16}+\widetilde{\gamma}_{26}+\widetilde{\gamma}_{36}+\widetilde{\gamma}_{46}+\Delta_{\delta_{4}}+2 k_{4}\right)} \cdot
\end{aligned}
$$

Next, we evaluate the remaining integrals via the Cauchy residue theorem. We close all $\widetilde{\gamma}_{r s}$ contours to the right to be able to drop the contribution from the arc at infinity, picking the lone semi-infinite sequence of poles starting at the origin, at $\widetilde{\gamma}_{r s}=j_{r s}$ for $j_{r s} \in \mathbb{Z}^{\geq 0}$ for each $(r s) \in \mathcal{V}_{7, \text { mix }}{ }^{18}$ These poles come from the poles of $\Gamma\left(-\widetilde{\gamma}_{r s}\right)$ in the first line of $(4.24)$, and

\footnotetext{
${ }^{17}$ Notably, after the $\gamma_{15}$ integral, we need to do a linear change of variables $\gamma_{14} \rightarrow \gamma_{14}-\gamma_{24}-\gamma_{34}$.

${ }^{18} \mathrm{We}$ recall that the contour for the $\widetilde{\gamma}_{r s}$ integrals was chosen such that it separates the semi-infinite series of poles running to the left from those running to the right; see (4.12).
} 
the residues, which are elementary to compute, introduce $\left(\begin{array}{c}n-2 \\ 2\end{array}\right)$ additional infinite sums:

$$
\begin{aligned}
I= & \left(\prod_{(r s) \in \mathcal{V}_{7, \text { mix }}} \sum_{j_{r s}=0}^{\infty} \frac{\left(1-v_{r s}\right)^{j_{r s}}}{j_{r s} !}\right) \\
& \times\left(\Gamma\left(\Delta_{12, \delta_{1}}-k_{1}\right) \Gamma\left(j_{24}+j_{26}+\Delta_{2 \delta_{1}, 1}+k_{1}\right) \Gamma\left(j_{13}+j_{14}+j_{15}+j_{16}+\Delta_{1 \delta_{1}, 2}+k_{1}\right)\right) \\
& \times\left(\Gamma\left(j_{14}+j_{15}+j_{16}+j_{24}+j_{26}+\Delta_{\delta_{1} \delta_{2}, 3}+k_{12,}\right) \Gamma\left(j_{13}+\Delta_{3 \delta_{1}, \delta_{2}}+k_{1,2}\right)\right. \\
& \left.\times \Gamma\left(j_{34}+j_{35}+j_{36}+\Delta_{3 \delta_{2}, \delta_{1}}+k_{2,1}\right)\right) \\
& \times\left(\Gamma\left(j_{14}+j_{15}+j_{24}+j_{34}+j_{35}+\Delta_{\delta_{2} \delta_{3}, \delta_{4}}+k_{23,4}\right) \Gamma\left(j_{46}+\Delta_{\delta_{3} \delta_{4}, \delta_{2}}+k_{34,2}\right)\right. \\
& \left.\times \Gamma\left(j_{16}+j_{26}+j_{36}+\Delta_{\delta_{2} \delta_{4}, \delta_{3}}+k_{24,3}\right)\right) \\
& \times\left(\Gamma\left(\Delta_{45, \delta_{3}}-k_{3}\right) \Gamma\left(j_{14}+j_{24}+j_{34}+\widetilde{\gamma}_{46}+\Delta_{4 \delta_{3}, 5}+k_{3}\right) \Gamma\left(\widetilde{\gamma}_{15}+\widetilde{\gamma}_{35}+\Delta_{5}\right) \Gamma\left(\Delta_{7 \delta_{4}, 6}+k_{4}\right) \Gamma\left(j_{16}+j_{26}+j_{36}+j_{46}+\Delta_{6 \delta_{4}, 7}+k_{4}\right)\right) \\
& \times \frac{1}{\Gamma\left(j_{13}+j_{14}+j_{15}+j_{16}+j_{24}+j_{26}+\Delta_{\delta_{1}}+2 k_{1}\right)} \\
& \times \frac{1}{\Gamma\left(j_{14}+j_{15}+j_{16}+j_{24}+j_{26}+j_{34}+j_{35}+j_{36}+\Delta_{\delta_{2}}+2 k_{2}\right)} \\
& \times \frac{1}{\Gamma\left(j_{14}+j_{15}+j_{24}+j_{34}+j_{35}+j_{46}+\Delta_{\delta_{3}}+2 k_{3}\right)} \\
& \times \frac{1}{\Gamma\left(j_{16}+j_{26}+j_{36}+j_{46}+\Delta_{\delta_{4}}+2 k_{4}\right)} \cdot
\end{aligned}
$$

Now we are ready to put everything together into (4.10) to obtain the conformal block $W_{7, \text { mix }}$. There will be a host of simplifying cancellations between factors of Gamma functions. For example, the factors of one-half times a Gamma function in the five Mellin vertex factors (4.18) cancel against a factor of one-half times a Gamma function appearing in each of the five OPE coefficients in (4.20) (after employing the explicit form of the OPE coefficients (4.21)). The remaining triplet of factors of Gamma functions of the form $\Gamma\left(\Delta_{a b, c}\right) \Gamma\left(\Delta_{b c, a}\right) \Gamma\left(\Delta_{c a, b}\right)$, in the numerators of each of the five OPE coefficients combine with the five groups of a triplet of Gamma functions in the numerator of (4.25) separated by parentheses, of the form $\Gamma\left(\Delta_{a b, c}+p\right) \Gamma\left(\Delta_{b c, a}+q\right) \Gamma\left(\Delta_{c a, b}+r\right)$, to give rise to five groups of triplets of Pochhammer symbols of the form $\left(\Delta_{a b, c}\right)_{p}\left(\Delta_{b c, a}\right)_{q}\left(\Delta_{c a, b}\right)_{r}$. These combine with the Lauricella functions in the Mellin vertex factors to give what we call the "conformal block vertex factors," one associated to each vertex of the canonical AdS diagram. The Gamma functions in the denominator of the five OPE coefficients with external dimensions in the argument cancel the same factors appearing in the normalization constant $\mathcal{N}$ in (4.6). For Gamma functions in the denominator of the OPE coefficients with internal dimensions in their arguments, there are precisely two copies for each internal dimension, while there is only one such factor for each internal dimension in the normalization constant $\mathcal{N}$. Thus after cancellations, we are left with a product in the numerator of $W_{7, \text { mix }}$ over factors of Gamma function, one each for every internal dimension. These combine with 
the Gamma functions in the denominator of (4.25) of the form $\Gamma\left(\Delta_{\delta_{i}}+t_{i}\right)$ to give rise to four Pochhammer symbols, $\left(\Delta_{\delta_{i}}\right)_{t_{i}}$ in the denominator. This together with the Pochhamer symbols originally appearing in (4.10) combine to give what we call the "conformal block edge factor," one for each internal leg of the canonical AdS diagram. Finally, the factor of $\pi^{(n-2) h}$ in $\mathcal{N}$ for $n=7$ cancels with $(n-2)$ factors of $\pi^{h}$ distributed over $(n-2)$ OPE coefficients (4.20). Thus all explicit factors of $\pi$ cancel.

This leads to the following final expression for the conformal block:

$$
W_{7, \text { mix }}\left(x_{i}\right)=W_{7, \text { mix }}^{0}\left(x_{i}\right)\left(\prod_{i=1}^{4} \sum_{k_{i}=0}^{\infty}\right)\left(\prod_{i=1}^{4} \frac{u_{i}^{\frac{\Delta_{\delta_{i}}}{2}+k_{i}}}{k_{i} !}\right)\left(\prod_{(r s) \in \mathcal{V}_{7, \text { mix }} j_{r_{s}}=0}^{\infty} \frac{\left(1-v_{r s}\right)^{j_{r s}}}{j_{r s} !}\right)\left(\prod_{i=1}^{4} E_{i}\right)\left(\prod_{i=1}^{5} V_{i}\right),
$$

where $E_{i}$ are precisely the conformal block edge factors (3.14) and $V_{i}$ are the conformal block vertex factors (3.16) prescribed by the Feynman rules, thus confirming the Feynman rules in this particular example.

\subsection{Comb channel}

To derive the comb channel conformal block obtained in section 3.2 via Feynman rules, we start with the Mellin amplitude of the following tree-level $n$-point AdS diagram $(n \geq 4)$ :

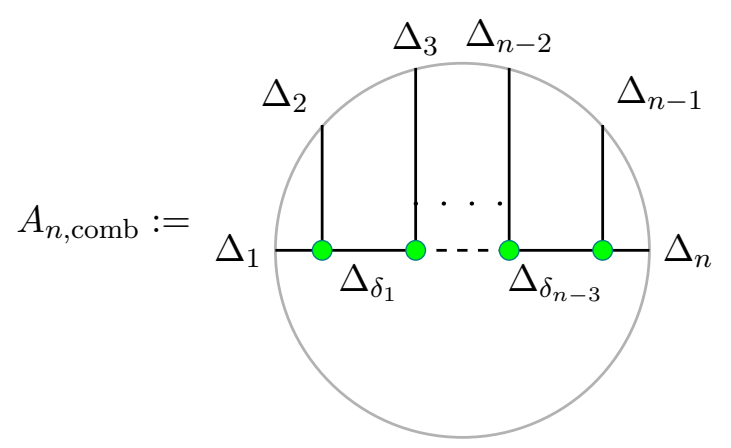

where the external scalar operators of dimensions $\Delta_{i}$ are inserted at coordinates $x_{i}$. In total $(n-3)$ single-particle bulk fields are exchanged in the interior, which are dual to single-trace operators with conformal dimensions $\Delta_{\delta_{i}}$ for $1 \leq i \leq n-3$. The ellipses in the middle indicate a repeating pattern. This is the canonical AdS diagram for the comb channel block shown in figure 1a.

To reproduce the block from section 3.2, we will utilize the same input data as before. This data comprises of a set of $n(n-3) / 2$ independent cross-ratios (3.17). Correspondingly, we choose the dependent Mellin index set $\mathcal{D}_{\text {comb }}$ to be (3.18) which allows us to rewrite the Mellin product (4.1) in terms of the cross-ratios and the Mandelstam invariants for internal legs (3.22), as shown in (3.20). The choice of cross-ratios also determines the index set $\mathcal{V}_{\text {comb }}(3.24)$, which represents a subset of independent Mellin variables. With $\mathcal{D}_{\text {comb }}$ and $\mathcal{V}_{\text {comb }}$ in hand, one can use (2.16) to obtain the index set associated with the remaining $(n-3)$ independent Mellin parameters,

$$
\mathcal{U}_{\text {comb }}:=\{(1 j) \mid 2 \leq j \leq n-2\} .
$$




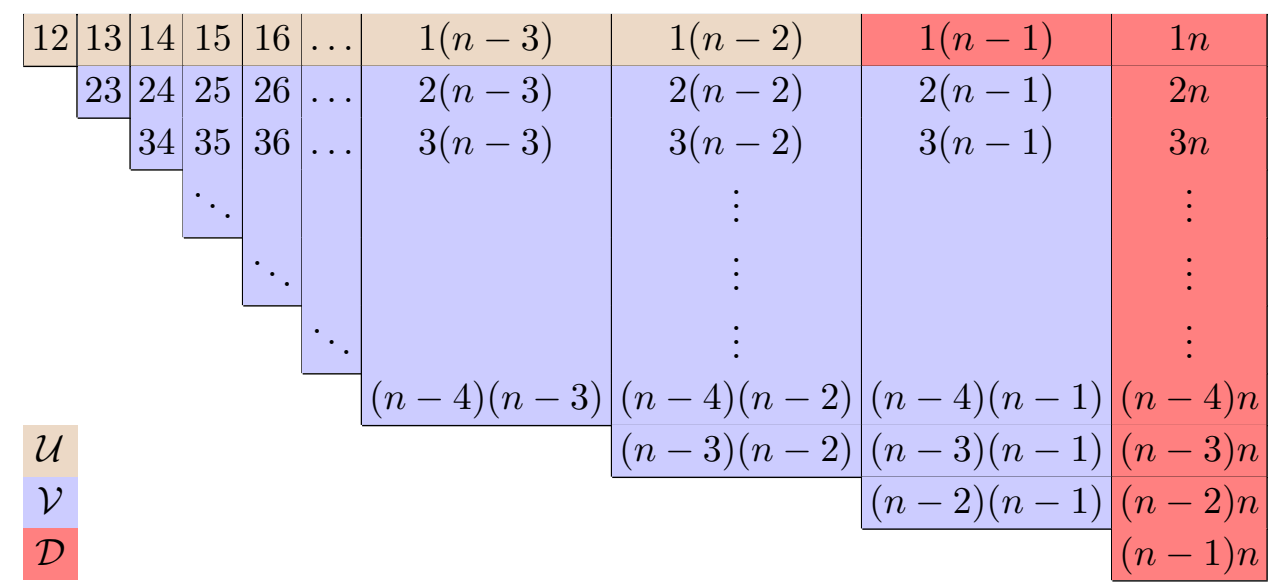

Figure 6. Color-coded matrix displaying the canonical choice of Mellin variable index subsets $\mathcal{U}_{\text {comb }}, \mathcal{V}_{\text {comb }}$ and $\mathcal{D}_{\text {comb }}$.

For reference, we have collected all comb channel index sets together into a color-coded upper-triangular matrix in figure 6.

Using the Feynman rules for Mellin amplitudes, it is trivial to write down Mellin amplitude for the diagram (4.27),

$$
\mathcal{M}_{n, \mathrm{comb}}\left(\gamma_{i j}\right)=\left(\prod_{i=1}^{n-3} \sum_{k_{i}=0}^{\infty}\right)\left(\prod_{i=1}^{n-3} E_{i}^{\mathrm{Mellin}}\right)\left(V_{1}^{\mathrm{Mellin}}\left(\prod_{i=1}^{n-4} V_{i+1}^{\mathrm{Mellin}}\right) V_{n-2}^{\mathrm{Mellin}}\right),
$$

where each of the $(n-3)$ edge factors is given in terms of the Mandelstam invariants $s_{i}$ and the associated single-trace parameters $k_{i}$ by $(4.4)$, and the $(n-2)$ vertex factors follow directly from (4.5):

$$
\begin{aligned}
V_{1}^{\text {Mellin }}= & \frac{1}{2} \Gamma\left(\Delta_{12 \delta_{1},}-h\right) F_{A}^{(1)}\left[\Delta_{12 \delta_{1}},-h ;\left\{-k_{1}\right\} ;\left\{\Delta_{\delta_{1}}-h+1\right\} ; 1\right] \\
V_{i+1}^{\text {Mellin }}= & \frac{1}{2} \Gamma\left(\Delta_{\delta_{i} \delta_{i+1}(i+2),}-h\right) \\
& \times F_{A}^{(2)}\left[\Delta_{\delta_{i} \delta_{i+1}(i+2)},-h ;\left\{-k_{i},-k_{i+1}\right\} ;\left\{\Delta_{\delta_{i}}-h+1, \Delta_{\delta_{i+1}}-h+1\right\} ; 1,1\right] \\
V_{n-2}^{\text {Mellin }}= & \frac{1}{2} \Gamma\left(\Delta_{(n-1) n \delta_{n-3}},-h\right) F_{A}^{(1)}\left[\Delta_{(n-1) n \delta_{n-3}},-h ;\left\{-k_{n-3}\right\} ;\left\{\Delta_{\delta_{n-3}}-h+1\right\} ; 1\right],
\end{aligned}
$$

for $1 \leq i \leq n-4$.

One can now obtain the single-trace projection of the AdS diagram (4.27) by evaluating

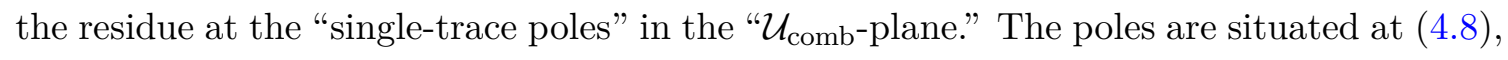
which in the $\mathcal{U}_{\text {comb-plane corresponds to }}$

$$
\gamma_{1 j}=\Delta_{j \delta_{j-2}, \delta_{j-1}}+k_{j-2, j-1}-\sum_{i=2}^{j-1} \gamma_{i j} \quad(2 \leq j \leq n-2),
$$

which we obtained by inverting (3.22), where we made the identifications $\Delta_{\delta_{0}}:=\Delta_{1}$ and $k_{0}:=0$ to write (4.31) compactly. 
The residue at the poles (4.31) is given by (4.9) where the dependent variables inside Gamma functions, written in (3.19), transform to

$$
\begin{aligned}
\gamma_{1(n-1)} & =\Delta_{\delta_{n-3}(n-1), n}+k_{n-3}-\sum_{j=2}^{n-2} \gamma_{j(n-1)} \\
\gamma_{1 n} & =\Delta_{1 n, 23 \ldots(n-1)}+\sum_{j=2}^{n-2} \gamma_{j(n-1)}+\sum_{2 \leq i<j \leq n-2} \gamma_{i j} \\
\gamma_{i n} & =\Delta_{i \delta_{i-1}, \delta_{i-2}}+k_{i-1, i-2}-\sum_{j=i+1}^{n-1} \gamma_{i j} \quad(2 \leq i \leq n-2) \\
\gamma_{(n-1) n} & =\Delta_{(n-1) n, \delta_{n-3}}-k_{n-3} .
\end{aligned}
$$

The conformal block (4.10) is then obtained by projecting out the following OPE coefficients:

$$
f_{1}=C_{\Delta_{1} \Delta_{2} \Delta_{\delta_{1}}} \quad f_{i+1}=C_{\Delta_{\delta_{i}} \Delta_{\delta_{i+1}} \Delta_{i+2}} \quad f_{n-2}=C_{\Delta_{n-1} \Delta_{n} \Delta_{\delta_{n-3}}}
$$

for $1 \leq i \leq n-4$, whose general form was given in (4.21).

We will now evaluate the second line of (4.10), or more precisely, the equivalent form in (4.14). Substituting (4.31) and (4.32) in (4.14), we get

$$
\begin{aligned}
I= & \left(\prod_{(r s) \in \mathcal{V}_{\text {comb }}} \int \frac{d \widetilde{\gamma}_{r s}}{2 \pi i} \int \frac{d \gamma_{r s}}{2 \pi i}\right)\left(\prod_{2 \leq r<s \leq n-1} \Gamma\left(-\widetilde{\gamma}_{r s}\right) \Gamma\left(\widetilde{\gamma}_{r s}+\gamma_{r s}\right)\left(v_{r s}-1\right)^{\widetilde{\gamma}_{r s}}\right) \\
& \times \Gamma\left(\Delta_{n 1,23 \ldots(n-1)}+\sum_{2 \leq r<s \leq n-1} \gamma_{r s}\right) \\
& \times\left(\prod_{i=2}^{n-1} \Gamma\left(\Delta_{i \delta_{i-2}, \delta_{i-1}}+k_{i-2, i-1}-\sum_{j=2}^{i-1} \gamma_{j i}\right) \Gamma\left(\Delta_{i \delta_{i-1}, \delta_{i-2}}+k_{i-1, i-2}-\sum_{j=i+1}^{n-1} \gamma_{i j}\right)\right),
\end{aligned}
$$

where we employed the additional identifications $\Delta_{\delta_{n-2}}:=\Delta_{n}$ and $k_{n-2}:=0$ to write $I$ compactly.

To evaluate the $\left(\begin{array}{c}n-2 \\ 2\end{array}\right)$-dimensional contour integral over $\gamma_{r s}$ variables, we will employ a multi-dimensional variant of the first Barnes lemma

$$
\begin{gathered}
\left(\prod_{r=1}^{K} \int \frac{d s_{r}}{2 \pi i} \Gamma\left(A_{r}+s_{r}\right) \Gamma\left(B_{r}-s_{r}\right)\right) \Gamma\left(C+\sum_{r=1}^{K} s_{r}\right) \Gamma\left(D-\sum_{r=1}^{K} s_{r}\right) \\
=\frac{\left(\prod_{r=1}^{K} \Gamma\left(A_{r}+B_{r}\right)\right) \Gamma(C+D) \Gamma\left(\sum_{r=1}^{K} A_{r}+D\right) \Gamma\left(\sum_{r=1}^{K} B_{r}+C\right)}{\Gamma\left(\sum_{r=1}^{K}\left(A_{r}+B_{r}\right)+C+D\right)}
\end{gathered}
$$

which can be easily proven by a repeated application of the first Barnes lemma (4.15). 
Looking forward, our strategy will be to evaluate the contour integrals in the order shown below (see figure 6 for color key):

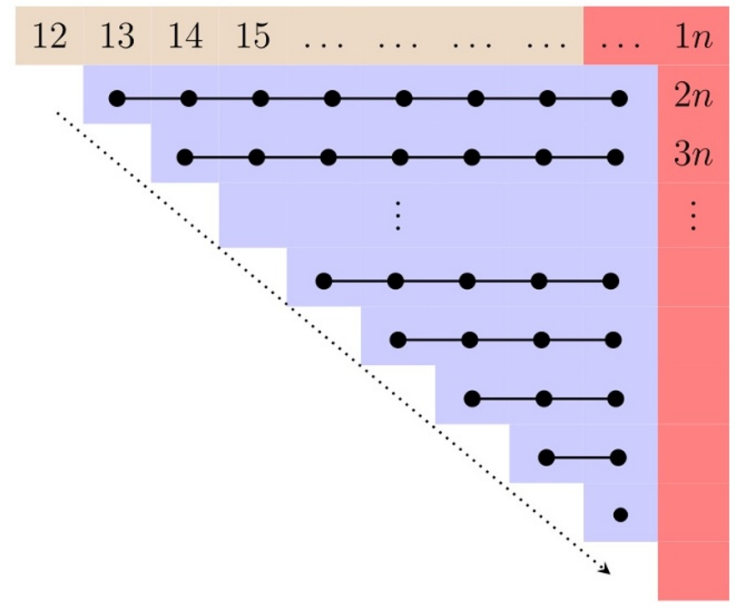

Here, integrals over elements of the index $\mathcal{V}_{\text {comb }}$ (shown in blue) are indicated with black dots. Integrals over all black dots connected via links are performed simultaneously by an application of (4.35), while the dotted arrow shows the order in which the contour integrals over the disconnected chains of dots are performed.

Concretely, for $2 \leq m \leq n-1$, define

$$
\begin{aligned}
J_{m}:= & \left(\prod_{m \leq r<s \leq n-1} \Gamma\left(\widetilde{\gamma}_{r s}+\gamma_{r s}\right)\right) \Gamma\left(\Delta_{n \delta_{m-2}, m(m+1) \ldots(n-1)}+k_{m-2}+\sum_{m \leq a<b \leq n-1} \gamma_{a b}\right) \\
& \times\left(\prod_{i=m}^{n-1} \Gamma\left(\sum_{j=2}^{m-1} \widetilde{\gamma}_{j i}+\Delta_{i \delta_{i-2}, \delta_{i-1}}+k_{i-2, i-1}-\sum_{j=m}^{i-1} \gamma_{j i}\right)\right) \\
& \times\left(\prod_{i=m}^{n-1} \Gamma\left(\Delta_{i \delta_{i-1}, \delta_{i-2}}+k_{i-1, i-2}-\sum_{j=i+1}^{n-1} \gamma_{i j}\right)\right)
\end{aligned}
$$

Then for $2 \leq m \leq n-2$ integrating $J_{m}$ over the elements in the $m$-th row of the index set $\mathcal{V}_{\text {comb }}$ in $(4.36)$ gives

$$
\left(\prod_{m+1 \leq j \leq n-1} \int \frac{d \gamma_{m j}}{2 \pi i}\right) J_{m}=\lambda_{m} J_{m+1}
$$

where

$$
\begin{aligned}
\lambda_{m}:= & \frac{\Gamma\left(\Delta_{m \delta_{m-2}, \delta_{m-1}}\right) \Gamma\left(\Delta_{m \delta_{m-1}, \delta_{m-2}}\right) \Gamma\left(\Delta_{\delta_{m-2} \delta_{m-1}, m}\right)}{\Gamma\left(\Delta_{\delta_{m-1}}\right)} \frac{1}{\left(\Delta_{\delta_{m-1}}\right)_{2 k_{m-1}+\sum_{i=2}^{m} \sum_{j=m+1}^{n-1} \widetilde{\gamma}_{i j}}} \\
& \times\left(\Delta_{m \delta_{m-2}, \delta_{m-1}}\right)_{k_{m-2, m-1}+\sum_{i=2}^{m-1} \tilde{\gamma}_{i m}\left(\Delta_{m \delta_{m-1}, \delta_{m-2}}\right)_{k_{m-1, m-2}}+\sum_{j=m+1}^{n-1} \tilde{\gamma}_{m j}} \\
& \times\left(\Delta_{\delta_{m-2} \delta_{m-1}, m}\right)_{k_{(m-2)(m-1)},+\sum_{i=2}^{m-1} \sum_{j=m+1}^{n-1} \tilde{\gamma}_{i j}},
\end{aligned}
$$


which is proven in appendix B.2. Moreover, at $m=n-1, J_{m}$ reduces to a $\gamma_{i j}$-independent expression,

$$
\begin{aligned}
J_{n-1}= & \Gamma\left(\Delta_{(n-1) \delta_{n-3}, n}\right) \Gamma\left(\Delta_{(n-1) n, \delta_{n-3}}\right) \Gamma\left(\Delta_{n \delta_{n-3},(n-1)}\right) \\
& \times\left(\Delta_{(n-1) \delta_{n-3}, n}\right)_{k_{n-3}+\sum_{i=2}^{n-2} \widetilde{\gamma}_{i(n-1)}}\left(\Delta_{(n-1) n, \delta_{n-3}}\right)_{-k_{n-3}}\left(\Delta_{n \delta_{n-3},(n-1)}\right)_{k_{n-3}} .
\end{aligned}
$$

The careful reader may notice that both (4.39) and (4.40) can be written more compactly purely in terms of Gamma functions. We have chosen to express them in terms of Pochhammer symbols to facilitate matching with the Feynman rules of section 2 at the end of this section.

In terms of $J_{m}$, the original contour integral (4.34) can be written as

$$
\begin{aligned}
I & =\left(\prod_{(r s) \in \mathcal{V}_{\text {comb }}} \int \frac{d \widetilde{\gamma}_{r s}}{2 \pi i} \Gamma\left(-\widetilde{\gamma}_{r s}\right)\left(v_{r s}-1\right)^{\widetilde{\gamma}_{r s}}\right)\left(\prod_{2 \leq i<j \leq n-1} \int \frac{d \gamma_{i j}}{2 \pi i}\right) J_{2} \\
& =\left(\prod_{(r s) \in \mathcal{V}_{\mathrm{comb}}} \int \frac{d \widetilde{\gamma}_{r s}}{2 \pi i} \Gamma\left(-\widetilde{\gamma}_{r s}\right)\left(v_{r s}-1\right)^{\widetilde{\gamma}_{r s}}\right) \lambda_{2}\left(\prod_{3 \leq i<j \leq n-1} \int \frac{d \gamma_{i j}}{2 \pi i}\right) J_{3} \\
& =\left(\prod_{(r s) \in \mathcal{V}_{\mathrm{comb}}} \int \frac{d \widetilde{\gamma}_{r s}}{2 \pi i} \Gamma\left(-\widetilde{\gamma}_{r s}\right)\left(v_{r s}-1\right)^{\widetilde{\gamma}_{r s}}\right) \lambda_{2} \lambda_{3}\left(\prod_{4 \leq i<j \leq n-1} \int \frac{d \gamma_{i j}}{2 \pi i}\right) J_{4} \\
& =\left(\prod_{(r s) \in \mathcal{V}_{\mathrm{comb}}} \int \frac{d \widetilde{\gamma}_{r s}}{2 \pi i} \Gamma\left(-\widetilde{\gamma}_{r s}\right)\left(v_{r s}-1\right)^{\widetilde{\gamma}_{r s}}\right) \lambda_{2} \lambda_{3} \ldots \lambda_{n-3}\left(\prod_{n-2 \leq i<j \leq n-1} \int \frac{d \gamma_{i j}}{2 \pi i}\right) J_{n-2} \\
& =\left(\prod_{(r s) \in \mathcal{V}_{\mathrm{comb}}} \int \frac{d \widetilde{\gamma}_{r s}}{2 \pi i} \Gamma\left(-\widetilde{\gamma}_{r s}\right)\left(v_{r s}-1\right)^{\tilde{\gamma}_{r s}}\right)\left(\prod_{m=2}^{n-2} \lambda_{m}\right) J_{n-1},
\end{aligned}
$$

where in the second step onward, we made repeated use of (4.38) to perform all $\gamma_{r s}$ integrals in the manner indicated in (4.36).

Now we turn to the $\widetilde{\gamma}_{r s}$ integrals. For carrying out the contour integrals, it is convenient to rewrite $I$ in terms of Gamma functions as

$$
\begin{aligned}
I= & \left(\prod_{(r s) \in \mathcal{V}_{\text {comb }}} \int \frac{d \widetilde{\gamma}_{r s}}{2 \pi i} \Gamma\left(-\widetilde{\gamma}_{r s}\right)\left(v_{r s}-1\right)^{\widetilde{\gamma}_{r s}}\right) \\
& \times\left(\prod_{m=2}^{n-2} \Gamma\left(\Delta_{\delta_{m-2} \delta_{m-1}, m}+k_{(m-2)(m-1)}+\sum_{i=2}^{m-1} \sum_{j=m+1}^{n-1} \widetilde{\gamma}_{i j}\right)\right. \\
& \left.\times \frac{\Gamma\left(\Delta_{m \delta_{m-2}, \delta_{m-1}}+k_{m-2, m-1}+\sum_{i=2}^{m-1} \widetilde{\gamma}_{i m}\right) \Gamma\left(\Delta_{m \delta_{m-1}, \delta_{m-2}}+k_{m-1, m-2}+\sum_{j=m+1}^{n-1} \widetilde{\gamma}_{m j}\right)}{\Gamma\left(\Delta_{\delta_{m-1}}+2 k_{m-1}+\sum_{i=2}^{m} \sum_{j=m+1}^{n-1} \widetilde{\gamma}_{i j}\right)}\right) \\
& \times \Gamma\left(\Delta_{(n-1) \delta_{n-3}, n}+k_{n-3}+\sum_{i=2}^{n-2} \widetilde{\gamma}_{i(n-1)}\right) \Gamma\left(\Delta_{(n-1) n, \delta_{n-3}}-k_{n-3}\right) \Gamma\left(\Delta_{n \delta_{n-3},(n-1)}+k_{n-3}\right) .
\end{aligned}
$$

Examining the pole structure of the integrand (4.42), we notice that just like in the example of the seven-point block in the previous subsection, we can evaluate the remaining $\widetilde{\gamma}_{r s}$ 
contour integrals by closing the contours to the right. In the process, each integral picks up a semi-infinite sequence of poles originating from $\Gamma\left(-\widetilde{\gamma}_{r s}\right)$ at $\widetilde{\gamma}_{r s}=j_{r s}$ for non-negative

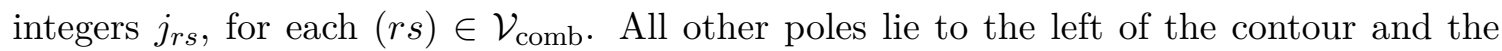
contribution from the arc at infinity vanishes. This immediately leads to

$$
\begin{aligned}
& I=\left(\prod_{(r s) \in \mathcal{V}_{\mathrm{Comb}}} \sum_{j_{r s}=0}^{\infty} \frac{\left(1-v_{r s}\right)^{j_{r s}}}{j_{r s} !}\right)\left(\prod_{m=2}^{n-2} \Gamma\left(\Delta_{\delta_{m-2} \delta_{m-1}, m}+k_{(m-2)(m-1)}+\sum_{r=2}^{m-1} \sum_{s=m+1}^{n-1} j_{r s}\right)\right. \\
& \left.\times \frac{\Gamma\left(\Delta_{m \delta_{m-2}, \delta_{m-1}}+k_{m-2, m-1}+\sum_{r=2}^{m-1} j_{r m}\right) \Gamma\left(\Delta_{m \delta_{m-1}, \delta_{m-2}}+k_{m-1, m-2}+\sum_{s=m+1}^{n-1} j_{m s}\right)}{\Gamma\left(\Delta_{\delta_{m-1}}+2 k_{m-1}+\sum_{r=2}^{m} \sum_{s=m+1}^{n-1} j_{r s}\right)}\right) \\
& \times \Gamma\left(\Delta_{(n-1) \delta_{n-3}, n}+k_{n-3}+\sum_{r=2}^{n-2} j_{r(n-1)}\right) \Gamma\left(\Delta_{(n-1) n, \delta_{n-3}}-k_{n-3}\right) \Gamma\left(\Delta_{n \delta_{n-3},(n-1)}+k_{n-3}\right) .
\end{aligned}
$$

It is now suggestive to re-express $I$ in terms of Pochhammer symbols, as shown here:

$$
\begin{aligned}
I= & \left(\prod_{(r s) \in \mathcal{V}_{\mathrm{Comb}}} \sum_{j_{r s}=0}^{\infty} \frac{\left(1-v_{r s}\right)^{j_{r s}}}{j_{r s} !}\right)\left(\prod_{m=2}^{n-2}\left(\Delta_{\delta_{m-2} \delta_{m-1}, m}\right)_{k_{(m-2)(m-1)},+\sum_{r=2}^{m-1} \sum_{s=m+1}^{n-1} j_{r s}}\right. \\
& \left.\times \frac{\left(\Delta_{m \delta_{m-2}, \delta_{m-1}}\right)_{k_{m-2, m-1}+\sum_{r=2}^{m-1} j_{r m}}\left(\Delta_{m \delta_{m-1}, \delta_{m-2}}\right)_{k_{m-1, m-2}+\sum_{s=m+1}^{n-1} j_{m s}}}{\left(\Delta_{\delta_{m-1}}\right)_{2 k_{m-1}+\sum_{r=2}^{m} \sum_{s=m+1}^{n-1} j_{r s}}}\right) \\
& \times\left(\Delta_{(n-1) \delta_{n-3}, n}\right)_{k_{n-3}+\sum_{r=2}^{n-2} j_{r(n-1)}}\left(\Delta_{(n-1) n, \delta_{n-3}}\right)_{-k_{n-3}}\left(\Delta_{n \delta_{n-3}, n-1}\right)_{k_{n-3}} \\
& \times\left(\prod_{m=2}^{n-2} \frac{\Gamma\left(\Delta_{\delta_{m-2} \delta_{m-1}, m}\right) \Gamma\left(\Delta_{m \delta_{m-2}, \delta_{m-1}}\right) \Gamma\left(\Delta_{m \delta_{m-1}, \delta_{m-2}}\right)}{\Gamma\left(\Delta_{\delta_{m-1}}\right)}\right) \\
& \times \Gamma\left(\Delta_{(n-1) \delta_{n-3}, n}\right) \Gamma\left(\Delta_{(n-1) n, \delta_{n-3}}\right) \Gamma\left(\Delta_{n \delta_{n-3}, n-1}\right) .
\end{aligned}
$$

Putting this $I$ back into the expression for the full conformal block (4.10), it is clear that, just like for the seven-point example above, all explicitly shown $(n-2)$ triplets of Gamma functions in the numerator of (4.44) cancel against the same triplets appearing in the $(n-2)$ OPE coefficients (4.33). The remaining factor of the Gamma function in the numerator of each OPE coefficient cancels against the Gamma function in each of the Mellin vertex factors (4.30). The explicit factor of Gamma function in the denominator in (4.44) cancels against one of two such identical factors in the normalization constant $\mathcal{N}$ written in (4.6), while the factors of Gamma functions in the denominators of the OPE coefficients cancel out all factors of Gamma functions in $\mathcal{N}$ which carry external conformal dimensions in their argument. All explicit factors of $\pi$ cancel out too, leaving only the Pochhammer symbols in (4.44), and powers of cross-ratios (as well as the expected factors of factorials).

It is now straightforward to check that the $(n-2)$ triplets of Pochhammer symbols in (4.44) reproduce precisely the $(n-2)$ triplets of Pochhammer symbols appearing in the vertex factors of the Feynman rules (3.34). Likewise the $(n-2)$ factors of Lauricella functions in the Mellin space vertex factors (4.30) appearing inside (4.10) find precise term by term agreement with the Lauricella functions in the conformal block vertex factors (3.34). The remaining $(n-3)$ ratios of Pochhamer symbols, of the form $\left(\Delta_{\delta_{i}}-h+1\right)_{k_{i}} /\left(\Delta_{\delta_{i}}\right)_{2 k_{i}+\ell_{i}}$ for an appropriately defined $\ell_{\delta_{i}}$ agree perfectly with the edge factors in the conformal 
block Feynman rules (2.4), where $\ell_{\delta_{i}}$ are identified as the post-Mellin parameters (3.30) associated with the internal legs labeled with conformal dimensions $\Delta_{\delta_{i}}$.

Thus, starting from first principles (viz. using the Feynman rules for Mellin amplitudes), we have reproduced the conformal block of section 3.2 which was obtained from an application of the proposed Feynman rules (and also previously obtained in ref. [58] using geodesic bulk diagram techniques).

\subsection{OPE channel}

To reproduce the OPE channel conformal block obtained previously via Feynman rules, we start with the following canonical AdS diagram:

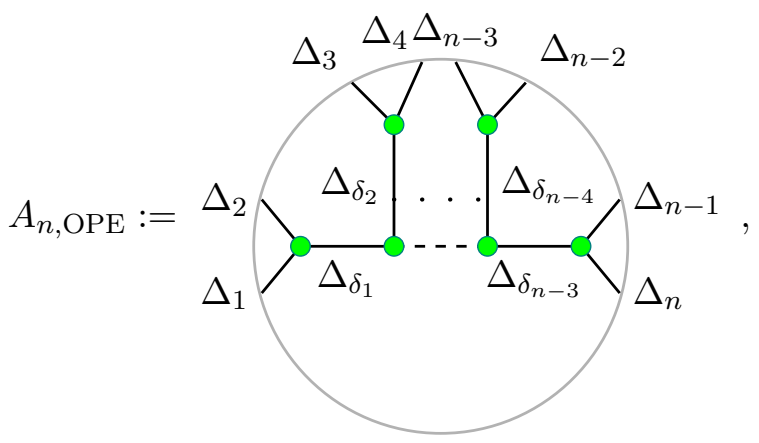

where the ellipses represent a repeating pattern of "upright Y-shaped" interacting legs attached to the central horizontal line. The vertical internal exchanges are labeled with even-indexed scaling dimensions $\Delta_{\delta_{2}}, \Delta_{\delta_{4}}, \Delta_{\delta_{6}}, \ldots, \Delta_{\delta_{n-4}}$, while the horizontal internal exchanges are labeled with odd-indexed scaling dimensions $\Delta_{\delta_{1}}, \Delta_{\delta_{3}}, \Delta_{\delta_{5}}, \ldots, \Delta_{\delta_{n-3}}$.

Just like in the previous two examples, we will use the same cross-ratios (3.35) as input data as used for Feynman rules. Let us recall the associated index sets which will be important in the computations to follow. The associated choice of dependent and independent Mellin index sets will also be identical. The dependent set $\mathcal{D}_{\text {OpE }}$ was given in (3.36) which allowed a rewriting of the Mellin product as shown in (3.39), with the Mandelstam invariants for each internal leg as defined in (3.38), and also determined the set $\mathcal{V}_{\mathrm{OPE}}$ as shown in (3.41). The remaining independent Mellin variables are associated with the set $\mathcal{U}_{\mathrm{OPE}}$ which can be found using $(2.16):^{19}$

$$
\mathcal{U}_{\mathrm{OPE}}:=\{((2 j+1)(2 j+2)) \mid 0 \leq j \leq n / 2-1\} \bigcup\{(2(2 j)) \mid 2 \leq j \leq n / 2-2\} .
$$

It is useful to represent the index sets visually as shown in figure 7 .

According to the Feynman rules for Mellin amplitudes, the AdS diagram (4.45) has the Mellin amplitude,

$$
\mathcal{M}_{n, \mathrm{OPE}}\left(\gamma_{i j}\right)=\left(\prod_{i=1}^{n-3} \sum_{k_{i}=0}^{\infty}\right)\left(\prod_{i=1}^{n-3} E_{i}^{\mathrm{Mellin}}\right)\left(\prod_{i=1}^{n / 2} V_{i}^{(1) \text { Mellin }}\right)\left(\prod_{i=1}^{n / 2-2} V_{i}^{(3) \text { Mellin }}\right) .
$$

\footnotetext{
${ }^{19}$ Explicitly, $\mathcal{U}_{\mathrm{OPE}}=\{(12),(34),(56),(78), \ldots,((n-1) n)\} \bigcup\{(24),(26),(28), \ldots,(2(n-4))\}$.
} 


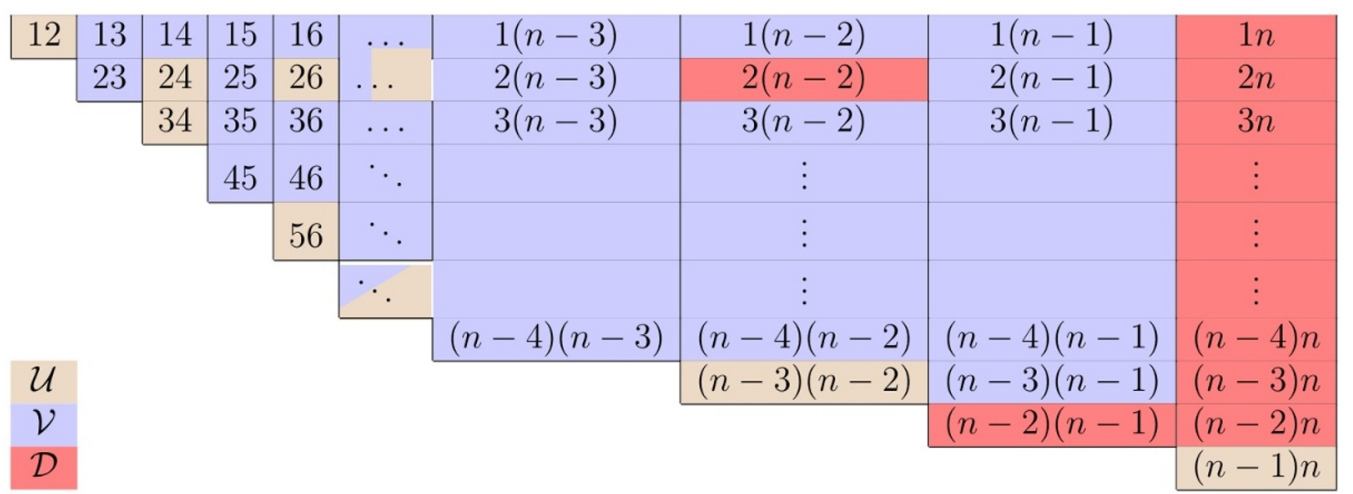

Figure 7. Color-coded matrix displaying the canonical choice of Mellin variable index subsets $\mathcal{U}_{\mathrm{OPE}}, \mathcal{V}_{\mathrm{OPE}}$ and $\mathcal{D}_{\mathrm{OPE}}$.

Here each of the edge factors is given by (4.4) with the Mandelstam invariants $s_{i}$ given in (3.38) and the associated legs assigned single-trace parameters $k_{i}$, and the vertex factors come directly from (4.5):

$$
\begin{aligned}
& V_{1}^{(1) \text { Mellin }}=\frac{1}{2} \Gamma\left(\Delta_{12 \delta_{1}},-h\right) F_{A}^{(1)}\left[\Delta_{12 \delta_{1}},-h ;\left\{-k_{1}\right\} ;\left\{\Delta_{\delta_{1}}-h+1\right\} ; 1\right] \\
& V_{a}^{(1) \text { Mellin }}=\frac{1}{2} \Gamma\left(\Delta_{(2 a-1)(2 a) \delta_{2 a-2}},-h\right) F_{A}^{(1)}\left[\Delta_{(2 a-1)(2 a) \delta_{2 a-2}},-h ;\left\{-k_{2 a-2}\right\} ;\left\{\Delta_{\delta_{2 a-2}}-h+1\right\} ; 1\right] \\
& V_{\frac{n}{2}}^{(1) \text { Mellin }}=\frac{1}{2} \Gamma\left(\Delta_{(n-1) n \delta_{n-3}},-h\right) F_{A}^{(1)}\left[\Delta_{(n-1) n \delta_{n-3}},-h ;\left\{-k_{n-3}\right\} ;\left\{\Delta_{\delta_{n-3}}-h+1\right\} ; 1\right] \\
& V_{b}^{(3) \text { Mellin }}=\frac{1}{2} \Gamma\left(\Delta_{\delta_{2 b-1} \delta_{2 b} \delta_{2 b+1}},-h\right) F_{A}^{(3)}\left[\Delta_{\delta_{2 b-1} \delta_{2 b} \delta_{2 b+1}},-h ;\left\{-k_{2 b-1},-k_{2 b},-k_{2 b+1}\right\} ;\right. \\
& \left.\left\{\Delta_{\delta_{2 b-1}}-h+1, \Delta_{\delta_{2 b}}-h+1, \Delta_{\delta_{2 b+1}}\right\} ; 1,1,1\right] \text {, }
\end{aligned}
$$

for $2 \leq a \leq n / 2-1$ and $1 \leq b \leq n / 2-2$.

Substituting (4.47) into (4.1), we proceed to obtain the single-trace projection of the AdS diagram as described around (4.8). This leads to (4.9) which is the desired conformal block times a set of known OPE coefficients,

$f_{1}^{(1)}=C_{\Delta_{1} \Delta_{2} \Delta_{\delta_{1}}} \quad f_{a}^{(1)}=C_{\Delta_{2 a-1} \Delta_{2 a} \Delta_{\delta_{2 a-2}}} \quad f_{\frac{n}{2}}^{(1)}=C_{\Delta_{n-1} \Delta_{n} \Delta_{\delta_{n-3}}} \quad f_{b}^{(3)}=C_{\Delta_{\delta_{2 b-1}} \Delta_{\delta_{2 b}} \Delta_{\delta_{2 b+1}}}$,

for $2 \leq a \leq n / 2-1$ and $1 \leq b \leq n / 2-2$, which can be factored out to obtain the block (4.10). This single-trace projection is obtained by evaluating the residue at the poles (4.8), which in the $\mathcal{U}_{\mathrm{OPE}}$-plane occur at

$$
\begin{aligned}
& \gamma_{12}=\Delta_{12, \delta_{1}}-k_{1} \quad \gamma_{(n-1) n}=\Delta_{(n-1) n, \delta_{n-3}}-k_{n-3} \quad \gamma_{(2 i+1)(2 i+2)}=\Delta_{(2 i+1)(2 i+2), \delta_{2 i}}-k_{2 i} \\
& \gamma_{2(2 j)}=\Delta_{\delta_{2 j-3} \delta_{2 j-2}, \delta_{2 j-1}}+k_{(2 j-3)(2 j-2),(2 j-1)}-\sum_{\substack{(a b) \in \mathcal{V}_{\mathrm{OPE}} \\
a<b, b=2 j-1 \text { or } 2 j}} \gamma_{a b} \text {, }
\end{aligned}
$$

for $1 \leq i \leq n / 2-2$, and $2 \leq j \leq n / 2-2$. Substituting these in the dependent Mellin 
variables $\gamma_{a b}(3.37)$ for $(a b) \in \mathcal{D}_{\mathrm{OPE}}$, we get $^{20}$

$$
\begin{aligned}
\gamma_{1 n} & =\Delta_{1 \delta_{1}, 2}+k_{1}-\sum_{j=3}^{n-1} \gamma_{1 j} \\
\gamma_{2 n} & =\Delta_{2 \delta_{n-3}, 1 \delta_{2} \delta_{4} \ldots \delta_{n-4}}+k_{(n-3), 24 \ldots(n-4)}-\gamma_{2(n-1)}+\sum_{j=3}^{n-2} \gamma_{1 j}+\sum_{\substack{3 \leq i<j \leq(n-2) \\
(i j) \in \mathcal{V}_{\mathrm{OPE}}}} \gamma_{i j} \\
\gamma_{(2 i+1) n} & =\Delta_{(2 i+1) \delta_{2 i},(2 i+2)}+k_{2 i}-\sum_{((2 i+1) b) \in \mathcal{V}_{\mathrm{OPE}}} \gamma_{(2 i+1) b} \\
\gamma_{(2 j+2) n} & =\Delta_{(2 j+2) \delta_{2 j+1},(2 j+1) \delta_{2 j-1}}+k_{2 j+1,2 j-1}+\sum_{\substack{(a(2 j+1)) \in \mathcal{V}_{\mathrm{OPE}} \\
a<2 j+1}} \gamma_{a(2 j+1)}-\sum_{\substack{((2 j+2) b) \in \mathcal{V}_{\mathrm{OPE}} \\
b>2 j+2}} \gamma_{(2 j+2) b} \\
\gamma_{(n-2) n} & =\Delta_{(n-2) n,(n-3)(n-1) \delta_{n-5}}-k_{n-5}+\sum_{j=1}^{n-3} \gamma_{j(n-1)}+\sum_{j=1}^{n-4} \gamma_{j(n-3)} \\
\gamma_{(n-2)(n-1)} & =\Delta_{(n-1) \delta_{n-3}, n}+k_{n-3}-\sum_{j=1}^{n-3} \gamma_{j(n-1)} \\
\gamma_{2(n-2)} & =\Delta_{\delta_{n-5} \delta_{n-4}, \delta_{n-3}}+k_{(n-5)(n-4),(n-3)}-\sum_{\substack{(a b) \in \mathcal{V}_{\mathrm{OPE}} \\
a<b, b=n-3 \text { or } n-2}} \gamma_{a b},
\end{aligned}
$$

for $1 \leq i \leq n / 2-2$ and $1 \leq j \leq n / 2-3$, where we made use of

$$
\sum_{j=2}^{\frac{n}{2}-2} \gamma_{2(2 j)}=\Delta_{\delta_{1} \delta_{2} \delta_{4} \delta_{6} \ldots \delta_{n-6}, \delta_{n-5}}+k_{1246 \ldots(n-6),(n-5)}-\sum_{\substack{(i j) \in \mathcal{V}_{\mathrm{OPE}} \\ i<j, j=3,4, \ldots, n-4}} \gamma_{i j}
$$

and

$$
\sum_{j=1}^{\frac{n}{2}-2} \gamma_{(2 j+1)(2 j+2)}=\Delta_{3456 \ldots(n-3)(n-2), \delta_{2} \delta_{4} \ldots \delta_{n-4}}-k_{24 \ldots(n-4),}
$$

Substituting (4.50) and (4.51) back in (4.14), we turn to evaluating the remaining contour integrals. Like in the seven-point and comb channel examples, we will first integrate over the $\gamma_{r s}$ variables for $(r s) \in \mathcal{V}_{\mathrm{OPE}}$. The order in which we will integrate is shown below

\footnotetext{
${ }^{20}$ A notational remark: if $(a b) \in \mathcal{V}_{\mathrm{OPE}}$, then so is $(b a) \in \mathcal{V}_{\mathrm{OPE}}$. So if $\{(14),(24),(34),(45),(46),(47)\} \subseteq$ $\mathcal{V}_{\mathrm{OPE}}$, whenever there is a restriction of the form $(a 4) \in \mathcal{V}_{\mathrm{OPE}}$ with $a<4$, it only admits elements from the set $\{(14),(24),(34)\}$ and not elements from the set $\{(45),(46),(47), \ldots\}$. Without the restriction $a<4$, all elements above will be admitted upon selecting $(a 4) \in \mathcal{V}_{\mathrm{OPE}}$.
} 
(consult figure 7 for reference):

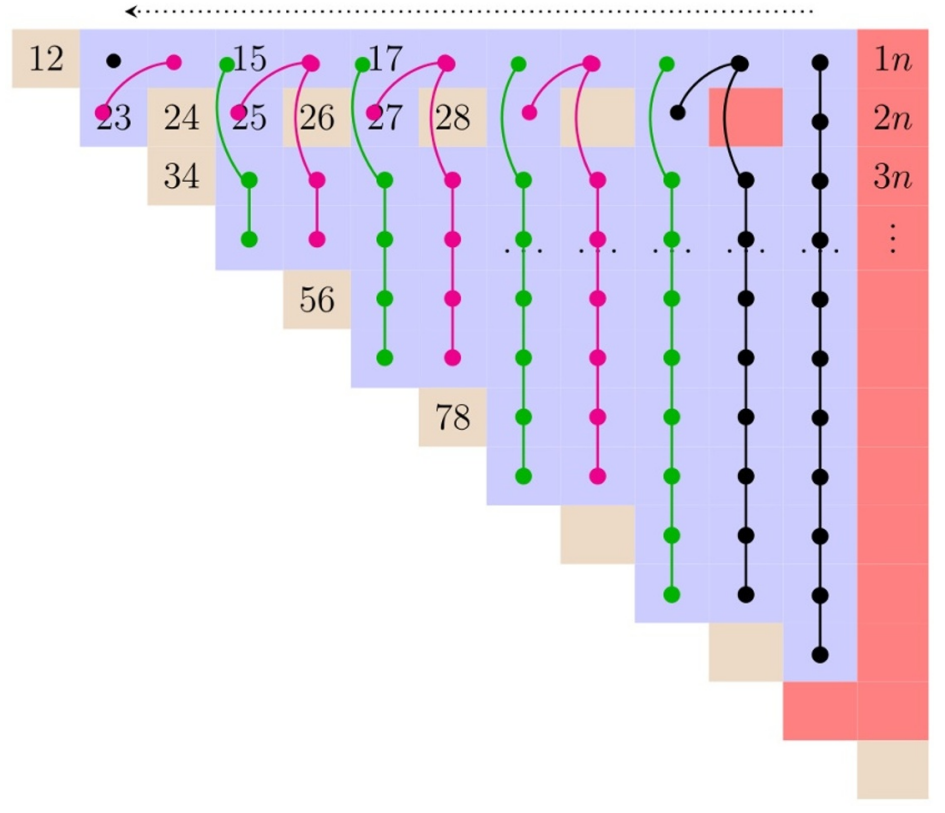

The direction of the dotted arrow (right to left) indicates the order in which we integrate over the connected elements of the set $\mathcal{V}_{\mathrm{OPE}}$. Each connected chain corresponds to a subset of contour integrals that will be evaluated with the help of the inductive first Barnes lemma (4.35). While any ordering works, the precise ordering chosen here makes it possible to set up an inductive step. The strategy will be as follows: we will first evaluate integrals (4.14) associated with the two (right-most) black-colored chains in (4.54). Using the resulting expression from the black-colored chain integrals, we will establish a two-step induction; the green and magenta colored chains above suggest how the induction will work.

In appendix B.3.1, we present the computation of the integrals marked as black-colored chains above. The end result of this computation is given in (B.24). To set up induction, we define a new contour integral $\widehat{I}_{n-2 K-1}$ such that

$$
\left.\widehat{I}_{n-2 K-1}\right|_{K=1}=I
$$

where $I$ is given by (B.24), and $\widehat{I}_{n-2 K-1}$ is defined to be

$$
\begin{aligned}
\widehat{I}_{n-2 K-1}:= & \left(\prod_{(r s) \in \mathcal{V}_{\mathrm{OPE}}} \int \frac{d \widetilde{\gamma}_{r s}}{2 \pi i} \Gamma\left(-\widetilde{\gamma}_{r s}\right)\left(v_{r s}-1\right)^{\widetilde{\gamma}_{r s}}\right)\left(\prod_{j=n-2 K-2}^{n-3} W_{j}\right) \\
& \times\left(\prod_{\substack{(r s) \in \mathcal{V}_{\mathrm{OPE}} \\
s \neq n-2 K-1, \ldots, n-1}} \int \frac{d \gamma_{r s}}{2 \pi i}\right) M_{n-2 K-1}\left(\prod_{r=1, r \neq 2}^{n-2 K-2} \int \frac{d \gamma_{r(n-2 K-1)}}{2 \pi i}\right) L_{n-2 K-1},
\end{aligned}
$$


for $1 \leq K \leq n / 2-2$. Here $W_{n-3}$ which was defined in (B.19), is repeated below,

$$
:=\frac{\Gamma\left(\Delta_{(n-1) n, \delta_{n-3}}-k_{n-3}\right) \Gamma\left(\Delta_{n \delta_{n-3},(n-1)}+k_{n-3}\right) \Gamma\left(\sum_{(i(n-1)) \in \mathcal{V}_{\mathrm{OPE}}} \widetilde{\gamma}_{i(n-1)}+\Delta_{(n-1) \delta_{n-3}, n}+k_{n-3}\right)}{\Gamma\left(\sum_{(i(n-1)) \in \mathcal{V}_{\mathrm{OPE}}} \widetilde{\gamma}_{i(n-1)}+\Delta_{\delta_{n-3}}+2 k_{n-3}\right)},
$$

and

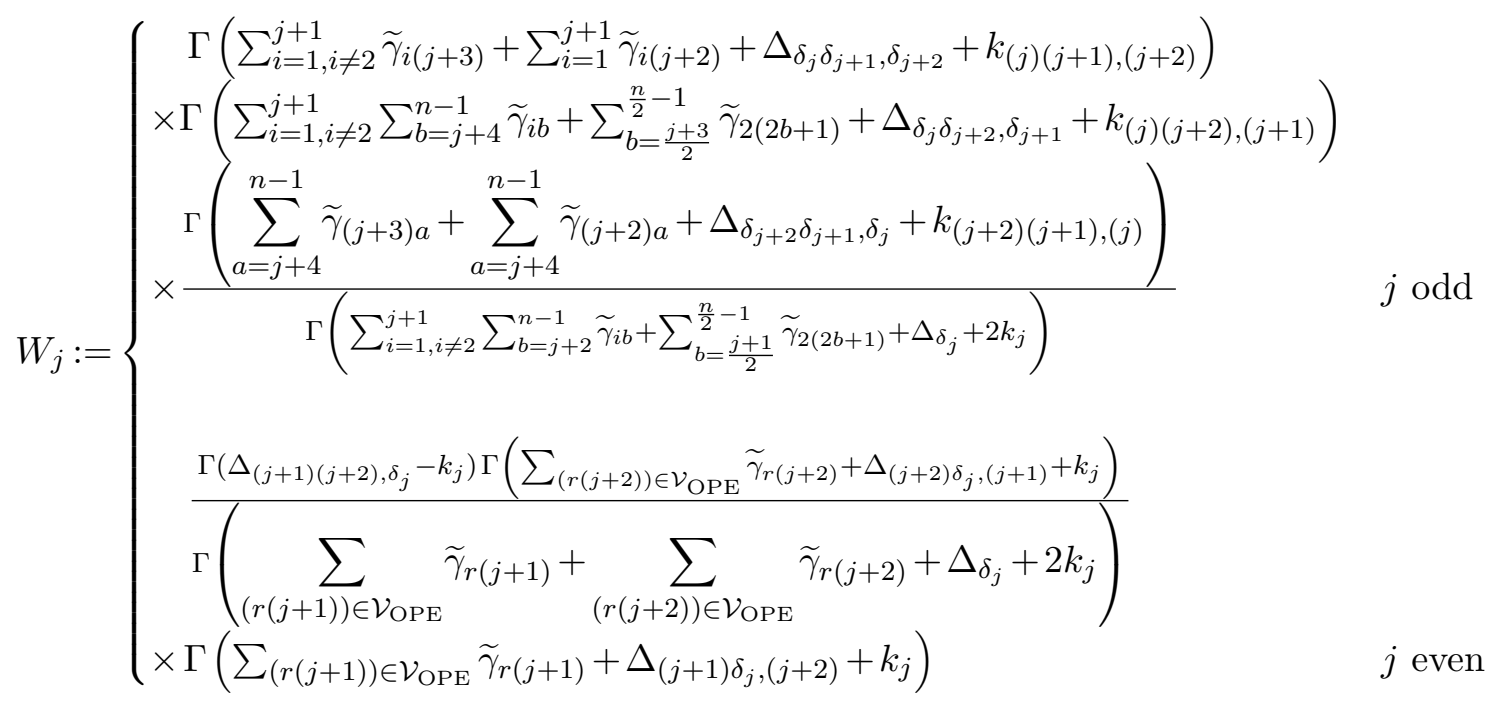

for $n-2 K-2 \leq j \leq n-4 .{ }^{21}$ Additionally, we define

$$
\begin{aligned}
& M_{n-2 K-1} \\
& :=\Gamma\left(\Delta_{12, \delta_{1}}-k_{1}\right)\left(\prod_{j=1}^{\frac{n}{2}-K-2} \Gamma\left(\Delta_{(2 j+1)(2 j+2), \delta_{2 j}}-k_{2 j}\right)\right)\left(\prod_{\substack{(r s) \in \mathcal{V}_{\mathrm{OPE}} \\
s \neq n-2 K-1, \ldots, n-1}} \Gamma\left(\widetilde{\gamma}_{r s}+\gamma_{r s}\right)\right) \\
& \times\left(\prod_{j=1}^{\frac{n}{2}-K-2} \Gamma\left(\Delta_{\delta_{2 j-1} \delta_{2 j}, \delta_{2 j+1}}+k_{(2 j-1)(2 j),(2 j+1)}-\sum_{\substack{(a b) \in \mathcal{V}_{\mathrm{OPE}} \\
a<b, b=2 j+1 \text { or } 2 j+2}} \gamma_{a b}\right)\right) \\
& \times \Gamma\left(\sum_{\substack{((n-2 K) a) \in \mathcal{V}_{\mathrm{OPE}} \\
a>n-2 K}} \tilde{\gamma}_{(n-2 K) a}+\sum_{\substack{((n-2 K-1) a) \in \mathcal{V}_{\mathrm{OPE}} \\
a>n-2 K}} \tilde{\gamma}_{(n-2 K-1) a}+\Delta_{\delta_{n-2 K-1} \delta_{n-2 K-2}, \delta_{n-2 K-3}}\right. \\
& \left.+k_{(n-2 K-1)(n-2 K-2),(n-2 K-3)}\right) \text {, }
\end{aligned}
$$

\footnotetext{
${ }^{21}$ We note that $W_{j}$ at $j=n-4$ coincides with (B.25).
} 
and

$$
\begin{aligned}
& L_{n-2 K-1} \\
& :=\left(\prod_{r=1, r \neq 2}^{n-2 K-2} \Gamma\left(\widetilde{\gamma}_{r(n-2 K)}+\widetilde{\gamma}_{r(n-2 K-1)}+\gamma_{r(n-2 K-1)}\right)\right) \\
& \times \Gamma\left(\sum_{j=n-2 K+1}^{n-1} \widetilde{\gamma}_{1 j}+\Delta_{1 \delta_{1}, 2}+k_{1}-\sum_{j=3}^{n-2 K-1} \gamma_{1 j}\right) \\
& \times \Gamma\left(\widetilde{\gamma}_{2(n-2 K-1)}+\Delta_{\delta_{n-2 K-3} \delta_{n-2 K-2}, \delta_{n-2 K-1}}+k_{(n-2 K-3)(n-2 K-2),(n-2 K-1)}-\sum_{a=1, a \neq 2}^{n-2 K-2} \gamma_{a(n-2 K-1)}\right) \\
& \times\left(\prod_{j=1}^{\frac{n}{2}-K-2} \Gamma\left(\sum_{a=n-2 K+1}^{n-1} \widetilde{\gamma}_{(2 j+1) a}+\Delta_{(2 j+1) \delta_{2 j},(2 j+2)}+k_{2 j}-\sum_{\substack{((2 j+1) b) \in \mathcal{V}_{\mathrm{OPE}} \\
b \neq n-2 K, \ldots, n-1}} \gamma_{(2 j+1) b}\right)\right) \\
& \times\left(\prod _ { j = 1 } ^ { \frac { n } { 2 } - K - 2 } \Gamma \left(\sum_{a=n-2 K+1}^{n-1} \widetilde{\gamma}_{(2 j+2) a}+\Delta_{(2 j+2) \delta_{2 j+1},(2 j+1) \delta_{2 j-1}}+k_{2 j+1,2 j-1}+\sum_{\substack{(b(2 j+1)) \in \mathcal{V}_{\mathrm{OPE}} \\
b<2 j+1}} \gamma_{b(2 j+1)}\right.\right. \\
& \left.\left.-\sum_{\substack{((2 j+2) b) \in \mathcal{V}_{\mathrm{OPE}} \\
b>2 j+2, b \neq n-2 K, \ldots, n-1}} \gamma_{(2 j+2) b}\right)\right) \\
& \times \Gamma\left(\sum_{i=0}^{K-1} \widetilde{\gamma}_{2(n-2 i-1)}+\Delta_{2 \delta_{n-2 K-1}, 1 \delta_{2} \delta_{4} \ldots \delta_{n-2 K-2}}+k_{(n-2 K-1), 24 \ldots(n-2 K-2)}+\sum_{j=3}^{n-2 K-1} \gamma_{1 j}\right. \\
& \left.+\sum_{\substack{3 \leq i<j \leq n-2 K-1 \\
(i j) \in \mathcal{V}_{\mathrm{OPE}}}} \gamma_{i j}\right)
\end{aligned}
$$

It can be checked that (4.55) holds. This will serve as the base case for an inductive argument which we develop next.

We would like to integrate $L_{n-2 K-1}$ over $\gamma_{1(n-2 K-1)}, \gamma_{3(n-2 K-1)}, \gamma_{4(n-2 K-1)}, \ldots$, $\gamma_{(n-2 K-2)(n-2 K-1)}$. This will turn out to be associated with integrating out a greencolored chain in (4.54) in an intermediate step, where this computation is described in 
appendix B.3.2. Here we rewrite the result of this computation, given in (B.31) as follows:

$$
\begin{aligned}
& \widehat{I}_{n-2 K-1} \\
&=\Gamma\left(\Delta_{12, \delta_{1}}-k_{1}\right)\left(\prod_{j=1}^{\frac{n}{2}-K-2} \Gamma\left(\Delta_{(2 j+1)(2 j+2), \delta_{2 j}}-k_{2 j}\right)\right)\left(\prod_{(r s) \in \mathcal{V}_{\mathrm{OPE}}} \int \frac{d \widetilde{\gamma}_{r s}}{2 \pi i} \Gamma\left(-\widetilde{\gamma}_{r s}\right)\left(v_{r s}-1\right)^{\widetilde{\gamma}_{r s}}\right) \\
& \times\left(\prod_{j=n-2 K-3}^{n-3} W_{j}\right)\left(\prod_{\substack{(r s) \in \mathcal{V}_{\mathrm{OPE}} \\
s \neq n-2 K-3, \ldots, n-1}} \int \frac{d \gamma_{r s}}{2 \pi i} \Gamma\left(\widetilde{\gamma}_{r s}+\gamma_{r s}\right)\right)\left(\prod_{r=1, r \neq 2}^{n-2 K-4} \int \frac{d \gamma_{r(n-2 K-3)}}{2 \pi i}\right) \\
& \times \int \frac{d \gamma_{2(n-2 K-3)}}{2 \pi i}\left(\prod_{r=1, r \neq 2}^{n-2 K-4} \int \frac{d \gamma_{r(n-2 K-2)}}{2 \pi i}\right) L_{n-2 K-2},
\end{aligned}
$$

where

$$
\begin{aligned}
& L_{n-2 K-2}:=\left(\prod_{\substack{(r s) \in \mathcal{V}_{\mathrm{OPE}} \\
r<s, s=n-2 K-3, n-2 K-2}} \Gamma\left(\widetilde{\gamma}_{r s}+\gamma_{r s}\right)\right) \\
& \times\left(\prod_{j=1}^{\frac{n}{2}-K-2} \Gamma\left(\Delta_{\delta_{2 j-1} \delta_{2 j}, \delta_{2 j+1}}+k_{(2 j-1)(2 j),(2 j+1)}-\sum_{\substack{(a b) \in \mathcal{V}_{\mathrm{OPE}} \\
a<b, b=2 j+1 \text { or } 2 j+2}} \gamma_{a b}\right)\right) \\
& \times \Gamma\left(\widetilde{\gamma}_{1(n-2 K)}+\widetilde{\gamma}_{1(n-2 K-1)}+\sum_{j=n-2 K+1}^{n-1} \widetilde{\gamma}_{1 j}+\Delta_{1 \delta_{1}, 2}+k_{1}-\sum_{j=3}^{n-2 K-2} \gamma_{1 j}\right) \\
& \times \Gamma\left(\sum_{i=0}^{K-1} \widetilde{\gamma}_{2(n-2 i-1)}+\widetilde{\gamma}_{2(n-2 K-1)}+\Delta_{2 \delta_{n-2 K-3}, 1 \delta_{2} \delta_{4} \ldots \delta_{n-2 K-4}}+k_{(n-2 K-3), 24 \ldots(n-2 K-4)}\right. \\
& \left.+\sum_{j=3}^{n-2 K-2} \gamma_{1 j}+\sum_{\substack{3 \leq i<j \leq n-2 K-2 \\
(i j) \in \mathcal{O P P E}}} \gamma_{i j}\right) \\
& \times\left(\prod_{j=1}^{\frac{n}{2}-K-2} \Gamma\left(\sum_{a=n-2 K-1}^{n-1} \widetilde{\gamma}_{(2 j+1) a}+\Delta_{(2 j+1) \delta_{2 j},(2 j+2)}+k_{2 j}-\sum_{\substack{((2 j+1) b) \in \mathcal{V}_{\mathrm{OPE}} \\
b \neq n-2 K-1, \ldots, n-1}} \gamma_{(2 j+1) b}\right)\right) \\
& \times\left(\prod _ { j = 1 } ^ { \frac { n } { 2 } - K - 2 } \Gamma \left(\sum_{a=n-2 K-1}^{n-1} \widetilde{\gamma}_{(2 j+2) a}+\Delta_{(2 j+2) \delta_{2 j+1},(2 j+1) \delta_{2 j-1}}+k_{2 j+1,2 j-1}\right.\right. \\
& \left.\left.+\sum_{\substack{(b(2 j+1)) \in \mathcal{V}_{\mathrm{OPE}} \\
b<2 j+1}} \gamma_{b(2 j+1)}-\sum_{\begin{array}{c}
((2 j+2) b) \in \mathcal{V}_{\mathrm{OPE}} \\
b>2 j+2, b \neq n-2 K-1, \ldots, n-1
\end{array}} \gamma_{(2 j+2) b}\right)\right) .
\end{aligned}
$$

Now, we would like to integrate over $\gamma_{2(n-2 K-3)}, \gamma_{1(n-2 K-2)}, \gamma_{3(n-2 K-2)}, \gamma_{4(n-2 K-2)}, \ldots$, $\gamma_{(n-2 K-4)(n-2 K-2)}$. This will be associated with integrating over the magenta-colored chain in (4.54) immediately to the left of the green-colored chain we previously integrated 
out. This computation is described in appendix B.3.3 and the final result is presented in (B.36). In fact, it is straightforward to show that (B.36) can be written as (4.56) upon sending $K \rightarrow K+1$. That is, comparing (B.36) with (4.56), we conclude,

$$
\widehat{I}_{n-2 K-1}=\left.\widehat{I}_{n-2 K-1}\right|_{K \rightarrow K+1}=\widehat{I}_{n-2 K-3}
$$

where on the r.h.s., $M_{n-2 K-3}$ and $L_{n-2 K-3}$ are given by (4.59) and (4.60) respectively, with $K \rightarrow K+1$, and the range of validity for $W_{j}$ in (4.58) now becomes $n-2 K-4 \leq j \leq n-4$. This establishes the inductive step, and together with the base case (4.55) furnishes the following chain of equalities:

$$
I=\widehat{I}_{n-3}=\widehat{I}_{n-5}=\cdots=\widehat{I}_{n-2 K-1}=\cdots=\widehat{I}_{3} .
$$

As we move progressively to the right down the chain of equalities above, we account for evaluations of more and more contour integrals from the set $\mathcal{V}_{\mathrm{OPE}}$, until we are left with just one integral. At the right-most equality at $K=\frac{n}{2}-2$, the original contour integral $I$ (see (B.12)) reduces to

$$
I=\widehat{I}_{3}=\left(\prod_{(r s) \in \mathcal{V}_{\mathrm{OPE}}} \int \frac{d \widetilde{\gamma}_{r s}}{2 \pi i} \Gamma\left(-\widetilde{\gamma}_{r s}\right)\left(v_{r s}-1\right)^{\widetilde{\gamma}_{r s}}\right)\left(\prod_{j=2}^{n-3} W_{j}\right) M_{3} \int \frac{d \gamma_{13}}{2 \pi i} L_{3}
$$

where the $W_{j}$ for $2 \leq j \leq n-3$ are given in (4.57)-(4.58), $M_{3}$ is given by (4.59) which at $K=\frac{n}{2}-2$ simplifies to

$$
M_{3}=\Gamma\left(\Delta_{12, \delta_{1}}-k_{1}\right) \Gamma\left(\sum_{\substack{(4 a) \in \mathcal{V}_{\mathrm{OPE}} \\ a>4}} \widetilde{\gamma}_{4 a}+\sum_{\substack{(3 a) \in \mathcal{V}_{\mathrm{OPE}} \\ a>4}} \widetilde{\gamma}_{3 a}+\Delta_{\delta_{3} \delta_{2}, \delta_{1}}+k_{32,1}\right)
$$

and $L_{3}$ is obtained by setting $K=\frac{n}{2}-2$ in (4.60),

$$
\begin{aligned}
L_{3}= & \Gamma\left(\widetilde{\gamma}_{14}+\widetilde{\gamma}_{13}+\gamma_{13}\right) \Gamma\left(\sum_{j=5}^{n-1} \widetilde{\gamma}_{1 j}+\Delta_{1 \delta_{1}, 2}+k_{1}-\gamma_{13}\right) \Gamma\left(\widetilde{\gamma}_{23}+\Delta_{\delta_{1} \delta_{2}, \delta_{3}}+k_{12,3}-\gamma_{13}\right) \\
& \times \Gamma\left(\sum_{i=0}^{\frac{n}{2}-3} \widetilde{\gamma}_{2(n-2 i-1)}+\Delta_{2 \delta_{3}, 1 \delta_{2}}+k_{3,2}+\gamma_{13}\right)
\end{aligned}
$$

The contour integral in (4.65), which corresponds to the lone black dot in (4.54) at the 
left-most extreme, can be evaluated using the first Barnes lemma (4.15), to give

$$
\begin{aligned}
I= & \left(\prod_{(r s) \in \mathcal{V}_{\mathrm{OPE}}} \int \frac{d \widetilde{\gamma}_{r s}}{2 \pi i} \Gamma\left(-\widetilde{\gamma}_{r s}\right)\left(v_{r s}-1\right)^{\widetilde{\gamma}_{r s}}\right)\left(\prod_{j=2}^{n-3} W_{j}\right) M_{3} \\
& \times \frac{\Gamma\left(\sum_{j=3}^{n-1} \widetilde{\gamma}_{1 j}+\Delta_{1 \delta_{1}, 2}+k_{1}\right) \Gamma\left(\widetilde{\gamma}_{13}+\widetilde{\gamma}_{14}+\widetilde{\gamma}_{23}+\Delta_{\delta_{1} \delta_{2}, \delta_{3}}+k_{12,3}\right)}{\Gamma\left(\sum_{j=3}^{n-1} \widetilde{\gamma}_{1 j}+\sum_{i=0}^{\frac{n}{2}-2} \widetilde{\gamma}_{2(n-2 i-1)}+\Delta_{\delta_{1}}+2 k_{1}\right)} \\
& \times \Gamma\left(\sum_{j=5}^{n-1} \widetilde{\gamma}_{1 j}+\sum_{i=0}^{\frac{n}{2}-3} \widetilde{\gamma}_{2(n-2 i-1)}+\Delta_{\delta_{1} \delta_{3}, \delta_{2}}+k_{13,2}\right) \Gamma\left(\sum_{i=0}^{\frac{n}{2}-2} \widetilde{\gamma}_{2(n-2 i-1)}+\Delta_{2 \delta_{1}, 1}+k_{1}\right) \\
\Rightarrow I= & \left(\prod_{(r s) \in \mathcal{V}_{\mathrm{OPE}}} \int \frac{d \widetilde{\gamma}_{r s}}{2 \pi i} \Gamma\left(-\widetilde{\gamma}_{r s}\right)\left(v_{r s}-1\right)^{\widetilde{\gamma}_{r s}}\right)\left(\prod_{j=1}^{n-3} W_{j}\right) \\
& \times \Gamma\left(\Delta_{12, \delta_{1}}-k_{1}\right) \Gamma\left(\sum_{j=3}^{n-1} \widetilde{\gamma}_{1 j}+\Delta_{1 \delta_{1}, 2}+k_{1}\right) \Gamma\left(\sum_{i=0}^{\frac{n}{2}-2} \widetilde{\gamma}_{2(n-2 i-1)}+\Delta_{2 \delta_{1}, 1}+k_{1}\right),
\end{aligned}
$$

where we identified a factor of $W_{1}$ above by comparing with (4.58) at $j=1$, thus extending the regime of validity of the $W_{j}$ coefficients in (4.57)-(4.58) to $1 \leq j \leq n-3$.

The $\left(\begin{array}{c}n-2 \\ 2\end{array}\right)$-dimensional contour integral over the $\widetilde{\gamma}_{r s}$ variables is significantly less complicated to evaluate. Just like in the seven-point and $n$-point comb channel examples, we close the contour to the right, and using the fact that all Gamma functions in the integrand contain positive linear combinations of $\widetilde{\gamma}_{r s}$ variables in their arguments except for the factors of $\Gamma\left(-\widetilde{\gamma}_{r s}\right)$, the only poles picked are the ones at origin and the semi-infinite sequence of poles at positive integral values of $\widetilde{\gamma}_{r s}$ for each $(r s) \in \mathcal{V}_{\mathrm{OPE}}$. This introduces $\left(\begin{array}{c}n-2 \\ 2\end{array}\right)$ new sums over the Mellin parameters $j_{r s}$, and furnishes a series expansion representation of the conformal block where, effectively, all positive linear combinations of the $\widetilde{\gamma}_{r s}$ Mellin variables in the Gamma functions get replaced with positive linear combinations of the corresponding Mellin parameters $j_{r s}$.

Let us mention some salient points of comparison between the blocks of this section and section 3.3. As shown in the previous two subsections, one can re-express all series coefficients in terms of Pochhammer symbols, such that all explicit factors of Gamma functions cancel out. The Lauricella functions in (4.48) and (3.48) are identical, and the positive linear combinations of Mellin parameters appearing in the Pochhammer symbols find perfect agreement as well. For instance, the arguments of the Gamma functions (or equivalently the Pochhammer symbols) in the numerators of $W_{j}$ for even $j=2 a-2$ match with those of the Pochhammer symbols in $V_{a}^{(1)}$ in (3.48) for $2 \leq a \leq n / 2-1$, and those of $W_{j}$ for odd $j=2 b-1$ match with those of $V_{b}^{(3)}$ in (3.48) for $1 \leq b \leq n / 2-2$. Moreover, the numerators of $W_{n-3}$ are identified with the Pochhammer symbols in $V_{n-2}^{(1)}$ in (3.48), while the triplet of factors in the final line of (4.68) are matched with the vertex factor $V_{1}^{(1)}$ in (3.48). This accounts for $(n-2)$ triplet of Pochhammer symbol combinations, one for each internal vertex of the binary graph. There are, additionally, $(n-3)$ factors of Pochhammer symbols in the denominators of the $(n-3) W_{j}$ coefficients, and each of these is in one-to-one correspondence with the denominators of the $(n-3)$ internal edge factors $(2.4)$. 
A careful comparison between the block found using the Feynman rules for conformal blocks, and the one found using the Mellin formalism in this section confirms that there is full agreement between the $n$-point conformal block of this section and section 3.3 , thus confirming the Feynman rules for $n$-point blocks in the OPE channel.

\section{Discussion}

In this paper we proposed a simple set of rules for constructing any scalar conformal block with scalar exchanges, given the appropriate cross-ratios as input data. The rules are summarized in (2.2)-(2.6), and the prescription for obtaining the post-Mellin parameters appearing in the Pochhammer symbols and summations is described in section 2.1. ${ }^{22}$ These rules help bypass lengthy, often impossibly hard computations needed to obtain conformal blocks. They are very similar and closely related to the Feynman rules for Mellin amplitudes, as in both methods we assign a factor for each edge and vertex appearing in the unrooted binary graph representation of the conformal block or Witten diagram. In fact, the same Lauricella functions appear in the Feynman rules for both conformal blocks and Mellin amplitudes. This was exhibited to be a consequence of the derivation of the Feynman rules in specific examples in section 4, where our starting point was the Mellin amplitude of certain canonical Witten diagrams. One important difference was that the type of Lauricella function which appears in a Mellin amplitude depends on the degree of the interaction vertex appearing in the AdS diagram; one associates the Lauricella function $F_{A}^{(\ell)}$ for a bulk interaction vertex with $\ell$ incident internal edges. For conformal blocks $\ell \leq 3$ because of the OPE structure of blocks; a related fact is that we needed to consider Mellin amplitudes for canonical Witten diagrams in an effective $\phi^{3}$ scalar field theory in AdS. The Lauricella functions were also expected from the point of view of previous work on the holographic duals of higher-point conformal blocks $[55,57,58]$, where the same functions appeared in the context of three-propagator identities which were used in the derivation of the geodesic diagram representation of blocks.

The Feynman rules provide an interesting, explicit and analytical representation for arbitrary conformal blocks, which may help investigate hidden mathematical structure and properties of conformal blocks, such as higher-point recursion relations, dimensional relations, and possible closed-form representations. The symmetric Lauricella functions in the power series expansion of the blocks also facilitate the symmetry analysis of conformal blocks; for instance permutation symmetries become manifest when the block is expressed in terms of the Lauricella functions. It would be interesting to undertake a detailed symmetry analysis, along the lines of ref. [30], of arbitrary conformal blocks as prescribed by the proposed Feynman rules. Recent work [63] on expressing higher-point functions in two and four spacetime dimensions in terms of generalizations of Lauricella systems in the configuration space of $n$ points, generalized to complex and quaternionic settings respectively, also provides an interesting mathematical connection and avenue of exploration.

The proposed Feynman rules were conjectured based on known results in the literature. We applied the rules to obtain the $n$-point block in the OPE channel which was not

\footnotetext{
${ }^{22}$ In section 4 , we also obtained a mixed series-integral representation (4.10).
} 
previously known, and verified it independently via the Mellin space formalism. Likewise we worked out a previously unknown seven-point block, both via Feynman rules and via Mellin amplitudes, and obtained a precise equivalence. These checks serve as non-trivial evidence in support of the proposed rules. The methodology in section 4 of proving the Feynman rules in particular examples, is also expected to work in exactly the same manner for any particular choice of conformal block beyond those considered in this paper. However, proving it for an arbitrary choice of a conformal block will presumably require more work. Nevertheless, it would be useful to prove these rules in generality for arbitrary blocks.

Finally, it should also be possible to generalize these rules to arbitrary-point conformal blocks for external and/or exchanged operators in arbitrary representations of the Lorentz group. This would be especially useful from the point of view of setting up an $n$-point conformal bootstrap for external scalars where internal exchanges can still involve spinning operators. Weight-shifting operators [25] and differential operators [32, 33] may be helpful in determining such generalizations. In fact, Mellin amplitudes for representations other than scalars (see e.g. refs. [69, 76-80]) may also inform the discussion on generalization of the conformal block Feynman rules beyond scalars. Turning the logic around, it would be interesting to investigate whether generalizations of the block Feynman rules to other representations benefit the study of higher-point spinning (Mellin) amplitudes.

\section{Acknowledgments}

The work of S. H. was partially supported by the SCS Summer Research Grant, by the Pomona RAISE Grant, and by Caltech's Visiting Undergraduate Research Program (VURP).

\section{A Lauricella functions}

The Lauricella function $F_{A}$ of $\ell$ variables is a generalized hypergeometric sum of $\ell$ variables [81-83] (see also ref. [69]) defined as

$$
F_{A}^{(\ell)}\left[g ;\left\{a_{1}, \ldots, a_{\ell}\right\} ;\left\{b_{1}, \ldots, b_{\ell}\right\} ; x_{1}, \ldots, x_{\ell}\right]:=\left[\prod_{i=1}^{\ell} \sum_{n_{i}=0}^{\infty}\right](g)_{\sum_{i=1}^{\ell} n_{i}} \prod_{i=1}^{\ell} \frac{\left(a_{i}\right)_{n_{i}}}{\left(b_{i}\right)_{n_{i}}} \frac{x_{i}^{n_{i}}}{n_{i} !} .
$$

One can always perform one of the sums in (A.1) to re-express $F_{A}^{(\ell)}$ in terms of functions involving $\ell-1$ summations. For example, we present some identities for $\ell \leq 3$ :

$$
\begin{aligned}
& F_{A}^{(1)}\left[\Delta_{a b c},-h ;\left\{-k_{c}\right\} ;\left\{\Delta_{c}-h+1\right\} ; 1\right]=\frac{\left(1-\Delta_{a b, c}\right)_{k_{c}}}{\left(\Delta_{c}-h+1\right)_{k_{c}}}, \\
& F_{A}^{(2)}\left[\Delta_{a b c,}-h ;\left\{-k_{b},-k_{c}\right\} ;\left\{\Delta_{b}-h+1, \Delta_{c}-h+1\right\} ; 1,1\right] \\
& =\frac{\left(1-\Delta_{a c, b}\right)_{k_{b}}\left(1-\Delta_{a b, c}\right)_{k_{c}}}{\left(\Delta_{b}-h+1\right)_{k_{b}}\left(\Delta_{c}-h+1\right)_{k_{c}}}{ }_{3} F_{2}\left[\left\{-k_{b},-k_{c}, \Delta_{a b c,}-h\right\} ;\left\{\Delta_{a c, b}-k_{b}, \Delta_{a b, c}-k_{c}\right\} ; 1\right],
\end{aligned}
$$


and

$$
\begin{aligned}
& F_{A}^{(3)}\left[\Delta_{a b c,-}-h ;\left\{-k_{a},-k_{b},-k_{c}\right\} ;\left\{\Delta_{a}-h+1, \Delta_{b}-h+1, \Delta_{c}-h+1\right\} ; 1,1,1\right] \\
&= \frac{(-1)^{k_{a}+k_{b}+k_{c}}}{\left(\Delta_{a}-h+1\right)_{k_{a}}\left(\Delta_{b}-h+1\right)_{k_{b}}\left(\Delta_{c}-h+1\right)_{k_{c}}} \\
& \times \frac{1}{\left(\Delta_{b c, a}\right)_{-k_{a}}\left(-\Delta_{b}+h\right)_{-k_{b}}\left(\Delta_{a b, c}\right)_{-k_{c}}} \\
& \times F_{2,1,0}^{1,3,2}\left[\begin{array}{c}
\Delta_{a b c,}-h ;-k_{b}, \Delta_{a b, c}, \Delta_{b c, a} ;-k_{a},-k_{c} \\
\Delta_{a b, c}-k_{c}, \Delta_{b c, a}-k_{a} ; \Delta_{b}-h+1 ;-
\end{array} \mid 1,1\right],
\end{aligned}
$$

where $F_{q, s, u}^{p, r, u}$ is the Kampé de Fériet function [82, 84] (see also ref. [61]), defined by the following hypergeometric series,

$$
F_{q, s, v}^{p, r, u}\left[\begin{array}{c}
\boldsymbol{a} ; \boldsymbol{c} ; \boldsymbol{f} \\
\boldsymbol{b} ; \boldsymbol{d} ; \boldsymbol{g}
\end{array} \mid x, y\right]:=\sum_{m, n=0}^{\infty} \frac{(\boldsymbol{a})_{m+n}(\boldsymbol{c})_{m}(\boldsymbol{f})_{n}}{(\boldsymbol{b})_{m+n}(\boldsymbol{d})_{m}(\boldsymbol{g})_{n}} \frac{x^{m} y^{n}}{m ! n !}
$$

where

$$
\begin{aligned}
(\boldsymbol{a})_{m+n} & :=\left(a_{1}\right)_{m+n} \cdots\left(a_{p}\right)_{m+n} & (\boldsymbol{b})_{m+n} & :=\left(b_{1}\right)_{m+n} \cdots\left(b_{q}\right)_{m+n} \\
(\boldsymbol{c})_{m} & :=\left(c_{1}\right)_{m} \cdots\left(c_{r}\right)_{m} & (\boldsymbol{d})_{m} & :=\left(d_{1}\right)_{m} \cdots\left(d_{s}\right)_{m} \\
(\boldsymbol{f})_{n} & :=\left(f_{1}\right)_{n} \cdots\left(f_{u}\right)_{n} & (\boldsymbol{g})_{n} & :=\left(g_{1}\right)_{n} \cdots\left(g_{v}\right)_{n} .
\end{aligned}
$$

\section{B Technical details}

\section{B.1 Proof of (3.46)}

In this appendix we will compute $\ell_{\delta_{2 i+1}}$ which satisfies the recursion relation

$$
\ell_{\delta_{2 i+3}} \stackrel{2 \mathcal{J}}{=} \ell_{\delta_{2 i+1}}+\ell_{\delta_{2 i+2}} \quad\left(0 \leq i \leq \frac{n}{2}-4\right)
$$

where the even-indexed $\ell_{\delta_{2 i+2}}$ and the smallest odd-indexed $\ell_{\delta_{1}}$ are known from (3.44).

We will now prove by induction that $\ell_{\delta_{2 i+1}}$ takes the form

$$
\ell_{\delta_{2 i+1}}=\sum_{s=2 i+3}^{n-1} j_{1 s}+\sum_{r=3}^{2 i+2} \sum_{s=2 i+3}^{n-1} j_{r s}+\sum_{z=i+1}^{\frac{n}{2}-1} j_{2(2 z+1)},
$$

for $0 \leq i \leq n / 2-3$.

Let's first establish the base case. For $i=1$,

$$
\begin{aligned}
\ell_{\delta_{3}} & \stackrel{2 \mathcal{J}}{=} \ell_{\delta_{1}}+\ell_{\delta_{2}} \\
& =\sum_{s=5}^{n-1} j_{1 s}+\sum_{r=3}^{4} \sum_{s=5}^{n-1} j_{r s}+\sum_{i=5,7,9, \ldots}^{n-1} j_{2 i}
\end{aligned}
$$

agrees with (B.2). 
For the inductive step, assume (B.2) is true for $i=K$, where $0 \leq K<n / 2-3$. We will now show that (B.2) holds for $i=K+1$. That is, assuming the following:

$$
\ell_{\delta_{2 K+1}}=\sum_{s=2 K+3}^{n-1} j_{1 s}+\sum_{r=3}^{2 K+2} \sum_{s=2 K+3}^{n-1} j_{r s}+\sum_{z=K+1}^{\frac{n}{2}-1} j_{2(2 z+1)}
$$

we can now compute

$$
\ell_{\delta_{2 K+3}} \stackrel{2 \mathcal{J}}{=} \ell_{\delta_{2 K+1}}+\ell_{\delta_{2 K+2}},
$$

where we can use

$$
\ell_{\delta_{2 K+2}}=\sum_{s=2 K+5}^{n-1} j_{(2 K+3) s}+\sum_{r=1}^{2 K+2} j_{r(2 K+3)}+j_{1(2 K+4)}+\sum_{s=2 K+5}^{n-1} j_{(2 K+4) s}+\sum_{r=3}^{2 K+2} j_{r(2 K+4)}
$$

and (B.4) to compute the 1.h.s. of (B.5):

$$
\begin{aligned}
\ell_{\delta_{2 K+3}} \stackrel{2 \mathcal{J}}{=} & \sum_{s=2 K+5}^{n-1} j_{(2 K+3) s}+\sum_{r=1}^{2 K+2} j_{r(2 K+3)}+j_{1(2 K+4)}+\sum_{s=2 K+5}^{n-1} j_{(2 K+4) s}+\sum_{r=3}^{2 K+2} j_{r(2 K+4)} \\
& +\sum_{s=2 K+3}^{n-1} j_{1 s}+\sum_{r=3}^{2 K+2} \sum_{s=2 K+3}^{n-1} j_{r s}+\sum_{z=K+1}^{\frac{n}{2}-1} j_{2(2 z+1)} \\
= & \sum_{s=2 K+3}^{n-1} j_{1 s}+\sum_{r=3}^{2 K+2} \sum_{s=2 K+3}^{n-1} j_{r s}+\sum_{z=K+1}^{\frac{n}{2}-1} j_{2(2 z+1)}-\sum_{2 K+3}^{2 K+4} j_{1 s}+\sum_{2 K+3}^{2 K+4} \sum_{2 K+5}^{n-1} j_{r s} \\
& -\sum_{r=3}^{2 K+2} \sum_{s=2 K+3}^{2 K+4} j_{r s}-j_{2(2 K+3)} \\
= & \sum_{s=2 K+5}^{n-1} j_{1 s}+\sum_{r=3}^{2 K+4} \sum_{s=2 K+5}^{n-1} j_{r s}+\sum_{z=K+2}^{n} j_{2(2 z+1)},
\end{aligned}
$$

which is the same as (B.2) for $i=K+1$, as needed.

\section{B.2 Proof of (4.38)}

We notice that the l.h.s. of (4.38) is of the form:

$$
K_{m}\left(\prod_{j=m+1}^{n-1} \int \frac{d s_{j}}{2 \pi i} \Gamma\left(A_{j}+s_{j}\right) \Gamma\left(B_{j}-s_{j}\right)\right) \Gamma\left(C+\sum_{j=m+1}^{n-1} s_{j}\right) \Gamma\left(D-\sum_{j=m+1}^{n-1} s_{j}\right),
$$

where the integration variables are $s_{i}=\gamma_{m i}$, and the coefficients are

$$
\begin{aligned}
A_{i} & =\widetilde{\gamma}_{m i} \\
B_{i} & =\sum_{j=2}^{m-1} \widetilde{\gamma}_{j i}+\Delta_{i \delta_{i-2}, \delta_{i-1}}+k_{i-2, i-1}-\sum_{j=m+1}^{i-1} \gamma_{j i} \\
C & =\Delta_{n \delta_{m-2}, m(m+1) \ldots(n-1)}+k_{m-2}+\sum_{m+1 \leq a<b \leq n-1} \gamma_{a b} \\
D & =\Delta_{m \delta_{m-1}, \delta_{m-2}}+k_{(m-1),(m-2)}
\end{aligned}
$$


with the overall factor

$$
\begin{aligned}
K_{m}= & \left(\prod_{m+1 \leq r \leq s \leq n-1} \Gamma\left(\widetilde{\gamma}_{r s}+\gamma_{r s}\right)\right) \Gamma\left(\sum_{i=2}^{m-1} \widetilde{\gamma}_{i m}+\Delta_{m \delta_{m-2}, \delta_{m-1}}+k_{m-2, m-1}\right) \\
& \times\left(\prod_{i=m+1}^{n-1} \Gamma\left(\Delta_{i \delta_{i-1}, \delta_{i-2}}+k_{i-1, i-2}-\sum_{j=i+1}^{n-1} \gamma_{i j}\right)\right) .
\end{aligned}
$$

Using the inductive Mellin Barnes lemma (4.35), we can easily evaluate (B.8) to obtain

$$
\begin{aligned}
& K_{m}\left(\prod_{i=m+1}^{n-1} \Gamma\left(\widetilde{\gamma}_{m i}+\sum_{j=2}^{m-1} \widetilde{\gamma}_{j i}+\Delta_{i \delta_{i-2}, \delta_{i-1}}+k_{i-2, i-1}-\sum_{j=m+1}^{i-1} \gamma_{j i}\right)\right) \\
& \times \Gamma\left(\Delta_{n \delta_{m-1},(m+1) \ldots(n-1)}+k_{m-1}+\sum_{m+1 \leq a<b \leq n-1} \gamma_{a b}\right) \\
& \times \Gamma\left(\sum_{i=m+1}^{n-1} \widetilde{\gamma}_{m i}+\Delta_{m \delta_{m-1}, \delta_{m-2}}+k_{(m-1),(m-2)}\right) \\
& \times \frac{\Gamma\left(\sum_{i=m+1}^{n-1} \sum_{j=2}^{m-1} \widetilde{\gamma}_{j i}+\Delta_{\delta_{m-1} \delta_{m-2}, m}+k_{m-2(m-1)}\right)}{\Gamma\left(\sum_{i=m+1}^{n-1} \sum_{j=2}^{m} \widetilde{\gamma}_{j i}+\Delta_{\delta_{m-1}}+2 k_{m-1}\right)} .
\end{aligned}
$$

Substituting in the explicit form for $K_{m}$ and using the definitions (4.37) and (4.39), we recognize (B.11) to be precisely the r.h.s. of (4.38).

\section{B.3 OPE channel calculations}

\section{B.3.1 Base case}

Our starting point is the contour integral (4.14), which takes the explicit form

$$
\begin{aligned}
& I=\Gamma\left(\Delta_{12, \delta_{1}}-k_{1}\right) \Gamma\left(\Delta_{(n-1) n, \delta_{n-3}}-k_{n-3}\right)\left(\prod_{j=1}^{\frac{n}{2}-2} \Gamma\left(\Delta_{(2 j+1)(2 j+2), \delta_{2 j}}-k_{2 j}\right)\right) \\
& \times\left(\prod_{(r s) \in \mathcal{V}_{\mathrm{OPE}}} \int \frac{d \widetilde{\gamma}_{r s}}{2 \pi i} \Gamma\left(-\widetilde{\gamma}_{r s}\right)\left(v_{r s}-1\right)^{\widetilde{\gamma}_{r s}}\right)\left(\prod_{(r s) \in \mathcal{V}_{\mathrm{OPE}}} \int \frac{d \gamma_{r s}}{2 \pi i} \Gamma\left(\widetilde{\gamma}_{r s}+\gamma_{r s}\right)\right) \\
& \times\left(\prod_{j=1}^{\frac{n}{2}-2} \Gamma\left(\Delta_{\delta_{2 j-1} \delta_{2 j}, \delta_{2 j+1}}+k_{(2 j-1)(2 j),(2 j+1)}-\sum_{\substack{(a b) \in \mathcal{V}_{\mathrm{OPE}} \\
a<b, b=2 j+1 \text { or } 2 j+2}} \gamma_{a b}\right)\right) \\
& \times \Gamma\left(\Delta_{1 \delta_{1}, 2}+k_{1}-\sum_{j=3}^{n-1} \gamma_{1 j}\right) \Gamma\left(\Delta_{(n-1) \delta_{n-3}, n}+k_{n-3}-\sum_{j=1}^{n-3} \gamma_{j(n-1)}\right) \\
& \times\left(\prod_{j=1}^{\frac{n}{2}-2} \Gamma\left(\Delta_{(2 j+1) \delta_{2 j},(2 j+2)}+k_{2 j}-\sum_{((2 j+1) b) \in \mathcal{V}_{\mathrm{OPE}}} \gamma_{(2 j+1) b}\right)\right) \\
& \times\left(\prod_{j=1}^{\frac{n}{2}-3} \Gamma\left(\Delta_{(2 j+2) \delta_{2 j+1},(2 j+1) \delta_{2 j-1}}+k_{2 j+1,2 j-1}+\sum_{\substack{(a(2 j+1)) \in \mathcal{V}_{\mathrm{OPE}} \\
a<2 j+1}} \gamma_{a(2 j+1)}-\sum_{\substack{((2 j+2) b) \in \mathcal{V}_{\mathrm{OPE}} \\
b>2 j+2}} \gamma_{(2 j+2) b}\right)\right)
\end{aligned}
$$




$$
\begin{aligned}
& \times \Gamma\left(\Delta_{2 \delta_{n-3}, 1 \delta_{2} \delta_{4} \ldots \delta_{n-4}}+k_{(n-3), 24 \ldots(n-4)}-\gamma_{2(n-1)}+\sum_{j=3}^{n-2} \gamma_{1 j}+\sum_{\substack{3 \leq i<j \leq n-2 \\
(i j) \in \mathcal{V} \mathrm{OPE}}} \gamma_{i j}\right) \\
& \times \Gamma\left(\Delta_{(n-2) n,(n-3)(n-1) \delta_{n-5}}-k_{n-5}+\sum_{j=1}^{n-3} \gamma_{j(n-1)}+\sum_{j=1}^{n-4} \gamma_{j(n-3)}\right) .
\end{aligned}
$$

Two straightforward applications of the first Barnes lemma (4.15) allow us to perform the $\gamma_{1(n-1)}$ and $\gamma_{2(n-1)}$ integrals. The result is

$$
\begin{aligned}
& I=\Gamma\left(\Delta_{12, \delta_{1}}-k_{1}\right) \Gamma\left(\Delta_{(n-1) n, \delta_{n-3}}-k_{n-3}\right)\left(\prod_{j=1}^{\frac{n}{2}-2} \Gamma\left(\Delta_{(2 j+1)(2 j+2), \delta_{2 j}}-k_{2 j}\right)\right) \\
& \times\left(\prod_{(r s) \in \mathcal{V}_{\mathrm{OPE}}} \int \frac{d \widetilde{\gamma}_{r s}}{2 \pi i} \Gamma\left(-\widetilde{\gamma}_{r s}\right)\left(v_{r s}-1\right)^{\widetilde{\gamma}_{r s}}\right)\left(\prod_{\substack{(r s) \in \mathcal{V}_{\mathrm{OPE}} \\
s \neq n-1}} \int \frac{d \gamma_{r s}}{2 \pi i} \Gamma\left(\widetilde{\gamma}_{r s}+\gamma_{r s}\right)\right) \\
& \times\left[\left(\prod_{r=3}^{n-3} \int \frac{d \gamma_{r(n-1)}}{2 \pi i}\right) \widetilde{L}_{n-1}\right]\left(\prod_{j=1}^{\frac{n}{2}-2} \Gamma\left(\Delta_{\delta_{2 j-1} \delta_{2 j}, \delta_{2 j+1}}+k_{(2 j-1)(2 j),(2 j+1)}-\sum_{\substack{(a b) \in \mathcal{V}_{\mathrm{OPE}} \\
a<b, b=2 j+1 \text { or } 2 j+2}} \gamma_{a b}\right)\right) \\
& \times \Gamma\left(\widetilde{\gamma}_{1(n-1)}+\Delta_{1 \delta_{1}, 2}+k_{1}-\sum_{j=3}^{n-2} \gamma_{1 j}\right) \Gamma\left(\Delta_{(n-2) \delta_{n-3},(n-3) \delta_{n-5}}+k_{n-3, n-5}+\sum_{j=1}^{n-4} \gamma_{j(n-3)}\right) \\
& \times \Gamma\left(\widetilde{\gamma}_{2(n-1)}+\Delta_{2 \delta_{n-3}, 1 \delta_{2} \delta_{4} \ldots \delta_{n-4}}+k_{(n-3), 24 \ldots(n-4)}+\sum_{j=3}^{n-2} \gamma_{1 j}+\sum_{\substack{3 \leq i<j \leq n-2 \\
(i j) \in \mathcal{V}_{\mathrm{OPE}}}} \gamma_{i j}\right) \\
& \times \Gamma\left(\sum_{i=1}^{2} \widetilde{\gamma}_{i(n-1)}+\Delta_{(n-2) \delta_{1},(n-3) \delta_{n-5} \delta_{2} \delta_{4} \ldots \delta_{n-4}}+k_{1,(n-5) 24 \ldots(n-4)}+\Delta_{\delta_{n-3}}+2 k_{n-3}\right. \\
& \left.+\sum_{j=1}^{n-4} \gamma_{j(n-3)}+\sum_{\substack{3 \leq i<j \leq n-2 \\
(i j) \in \mathcal{V}_{\mathrm{OPE}}}} \gamma_{i j}\right)^{-1},
\end{aligned}
$$


where the integrand for the integrals over $\gamma_{3(n-1)}, \ldots, \gamma_{(n-3)(n-1)}$ above has been collected into the following object,

$$
\begin{aligned}
& \widetilde{L}_{n-1} \\
& :=\left(\prod_{r=3}^{n-3} \Gamma\left(\widetilde{\gamma}_{r(n-1)}+\gamma_{r(n-1)}\right)\right)\left(\prod_{j=1}^{\frac{n}{2}-2} \Gamma\left(\Delta_{(2 j+1) \delta_{2 j},(2 j+2)}+k_{2 j}-\sum_{((2 j+1) b) \in \mathcal{V}_{\mathrm{OPE}}} \gamma_{(2 j+1) b}\right)\right) \\
& \times\left(\prod_{j=1}^{\frac{n}{2}-3} \Gamma\left(\Delta_{(2 j+2) \delta_{2 j+1},(2 j+1) \delta_{2 j-1}}+k_{2 j+1,2 j-1}+\sum_{\substack{(a(2 j+1)) \in \mathcal{V}_{\mathrm{OPE}} \\
a<2 j+1}} \gamma_{a(2 j+1)}-\sum_{\substack{((2 j+2) b) \in \mathcal{V}_{\mathrm{OPE}} \\
b>2 j+2}} \gamma_{(2 j+2) b}\right)\right) \\
& \times \Gamma\left(\Delta_{(n-2) n \delta_{1} \delta_{n-3},(n-3)(n-1) \delta_{n-5} \delta_{2} \delta_{4} \ldots \delta_{n-4}}+k_{1(n-3),(n-5) 24 \ldots(n-4)}+\sum_{j=1}^{n-4} \gamma_{j(n-3)}+\sum_{\substack{3 \leq i<j \leq n-2 \\
(i j) \in \mathcal{V}_{\mathrm{OPE}}}} \gamma_{i j}\right. \\
& \left.\quad+\sum_{j=3}^{n-3} \gamma_{j(n-1)}\right) \Gamma\left(\sum_{i=1}^{2} \widetilde{\gamma}_{i(n-1)}+\Delta_{(n-1) \delta_{n-3}, n}+k_{n-3}-\sum_{j=3}^{n-3} \gamma_{j(n-1)}\right) .
\end{aligned}
$$

To evaluate the integrals over $\widetilde{L}_{n-1}$, we can use the inductive first Barnes lemma (4.35) with the identifications

$$
\begin{aligned}
& A_{r}=\widetilde{\gamma}_{(r+2)(n-1)} \\
& B_{r}= \begin{cases}\Delta_{(r+2) \delta_{r+1},(r+3)}+k_{r+1}-\sum_{\substack{((r+2) b) \in \mathcal{V}_{\mathrm{OPE}} \\
b \neq n-1}} \gamma_{(r+2) b} & (r \text { odd }) \\
\Delta_{(r+2) \delta_{r+1},(r+1) \delta_{r-1}}+k_{r+1, r-1}+\sum_{\substack{(a(r+1)) \in \mathcal{V} \\
a<r+1}} \gamma_{a(r+1)}-\sum_{\substack{((r+2) b) \in \mathcal{V}_{\mathrm{OPE}} \\
b>r+2, b \neq n-1}} \gamma_{(r+2) b} \quad(r \text { even })\end{cases} \\
& C=\Delta_{(n-2) n \delta_{1} \delta_{n-3},(n-3)(n-1) \delta_{n-5} \delta_{2} \delta_{4} \ldots \delta_{n-4}}+k_{1(n-3),(n-5) 24 \ldots(n-4)}+\sum_{j=1}^{n-4} \gamma_{j(n-3)}+\sum_{\substack{3 \leq i<j \leq n-2 \\
(i j) \in \mathcal{V}_{\mathrm{OPE}}}} \gamma_{i j} \\
& D=\sum_{i=1}^{2} \widetilde{\gamma}_{i(n-1)}+\Delta_{(n-1) \delta_{n-3}, n}+k_{n-3}
\end{aligned}
$$


for $1 \leq r \leq n-5$. Then the contour integrals evaluate to

$$
\begin{aligned}
& \left(\prod_{r=3}^{n-3} \int \frac{d \gamma_{r(n-1)}}{2 \pi i}\right) \widetilde{L}_{n-1} \\
& =\frac{\Gamma\left(\Delta_{(n-2) \delta_{1},(n-3) \delta_{n-5} \delta_{2} \delta_{4} \ldots \delta_{n-4}}+k_{1,(n-5) 24 \ldots(n-4)}+\sum_{j=1}^{n-4} \gamma_{j(n-3)}+\sum_{\substack{3 \leq i<j \leq n-2 \\
(i j) \in \mathcal{V}_{\mathrm{OPE}}}} \gamma_{i j}+\sum_{i=1}^{2} \tilde{\gamma}_{i(n-1)}+\Delta_{\delta_{n-3}}+2 k_{n-3}\right)}{\Gamma\left(\sum_{r=1}^{n-5} \tilde{\gamma}_{(r+2)(n-1)}+\sum_{i=1}^{2} \tilde{\gamma}_{i(n-1)}+\Delta_{\delta_{n-3}}+2 k_{n-3}\right)} \\
& \times\left(\prod_{r=1,3, \ldots}^{n-5} \Gamma\left(\widetilde{\gamma}_{(r+2)(n-1)}+\Delta_{(r+2) \delta_{r+1},(r+3)}+k_{r+1}-\sum_{\substack{((r+2) b) \in \mathcal{V}_{\mathrm{OPE}} \\
b \neq n-1}} \gamma_{(r+2) b}\right)\right. \\
& \times\left(\prod_{r=2,4, \ldots}^{n-6} \Gamma \widetilde{\gamma}_{(r+2)(n-1)}+\Delta_{(r+2) \delta_{r+1},(r+1) \delta_{r-1}}+k_{r+1, r-1}+\sum_{\substack{(a(r+1)) \in \mathcal{V}_{\mathrm{OPE}} \\
a<r+1}} \gamma_{a(r+1)}-\sum_{\substack{((r+2) b) \in \mathcal{V}_{\mathrm{OPE}} \\
b>r+2, b \neq n-1}} \gamma_{(r+2) b}\right) \\
& \times \Gamma\left(\Delta_{n \delta_{n-3},(n-1)}+k_{(n-3)}\right) \Gamma\left(\sum_{r=1}^{n-5} \widetilde{\gamma}_{(r+2)(n-1)}+\sum_{i=1}^{2} \widetilde{\gamma}_{i(n-1)}+\Delta_{(n-1) \delta_{n-3}, n}+k_{n-3}\right)
\end{aligned}
$$

where we used

$$
\sum_{r=1}^{n-5} B_{r}=\Delta_{(n-3) \delta_{2} \delta_{4} \delta_{6} \ldots \delta_{n-6} \delta_{n-4} \delta_{n-5},(n-2) \delta_{1}}+k_{246 \ldots(n-6)(n-4)(n-5), 1}-\sum_{\substack{3 \leq i<j \leq n-2 \\(i j) \in \mathcal{V} \text { OPE }}} \gamma_{i j}-\sum_{j=1}^{n-4} \gamma_{j(n-3)} .
$$

Putting this result back in $I$, we obtain

$$
\begin{aligned}
& I=\Gamma\left(\Delta_{12, \delta_{1}}-k_{1}\right)\left(\prod_{j=1}^{\frac{n}{2}-2} \Gamma\left(\Delta_{(2 j+1)(2 j+2), \delta_{2 j}}-k_{2 j}\right)\right)\left(\prod_{(r s) \in \mathcal{V}_{\mathrm{OPE}}} \int \frac{d \widetilde{\gamma}_{r s}}{2 \pi i} \Gamma\left(-\widetilde{\gamma}_{r s}\right)\left(v_{r s}-1\right)^{\widetilde{\gamma}_{r s}}\right) \\
& \times\left(\prod_{\substack{(r s) \in \mathcal{V}_{\mathrm{OPE}} \\
s \neq n-1, n-2, n-3}} \int \frac{d \gamma_{r s}}{2 \pi i} \Gamma\left(\widetilde{\gamma}_{r s}+\gamma_{r s}\right)\right)\left(\prod_{\substack{(r s) \in \mathcal{V}_{\mathrm{OPE}} \\
s=n-3, r<s, r \neq 2}} \int \frac{d \gamma_{r s}}{2 \pi i}\right) W_{n-3} \\
& \times\left(\prod_{j=1}^{\frac{n}{2}-3} \Gamma\left(\Delta_{\delta_{2 j-1} \delta_{2 j}, \delta_{2 j+1}}+k_{(2 j-1)(2 j),(2 j+1)}-\sum_{\substack{(a b) \in \mathcal{V}_{\mathrm{OPE}} \\
a<b, b=2 j+1 \text { or } 2 j+2}} \gamma_{a b}\right)\right) \\
& \times\left(\prod_{\substack{(r s) \in \mathcal{V}_{\mathrm{OPE}} \\
s=n-2 \text { or }(r s)=(2(n-3))}} \int \frac{d \gamma_{r s}}{2 \pi i}\right) L_{n-2},
\end{aligned}
$$


where we have defined

$$
:=\frac{\Gamma\left(\Delta_{(n-1) n, \delta_{n-3}}-k_{n-3}\right) \Gamma\left(\Delta_{n \delta_{n-3},(n-1)}+k_{n-3}\right) \Gamma\left(\sum_{(i(n-1)) \in \mathcal{V}_{\mathrm{OPE}}} \tilde{\gamma}_{i(n-1)}+\Delta_{(n-1) \delta_{n-3}, n}+k_{n-3}\right)}{\Gamma\left(\sum_{(i(n-1)) \in \mathcal{V}_{\mathrm{OPE}}} \tilde{\gamma}_{i(n-1)}+\Delta_{\delta_{n-3}}+2 k_{n-3}\right)},
$$

and

$$
\begin{aligned}
& L_{n-2} \\
& :=\left(\prod_{\substack{(r s) \in \mathcal{V}_{O P E} \\
r<s, s=n-2, n-3}} \Gamma\left(\widetilde{\gamma}_{r s}+\gamma_{r s}\right)\right) \\
& \times \Gamma\left(\widetilde{\gamma}_{1(n-1)}+\Delta_{1 \delta_{1}, 2}+k_{1}-\sum_{j=3}^{n-2} \gamma_{1 j}\right) \Gamma\left(\Delta_{\delta_{n-5} \delta_{n-4}, \delta_{n-3}}+k_{(n-5)(n-4),(n-3)}-\sum_{\substack{(a b) \in \mathcal{V}_{\mathrm{OPE}} \\
a<b, b=n-3}} \gamma_{a b}\right) \\
& \times\left(\prod_{j=1}^{\frac{n}{2}-2} \Gamma\left(\widetilde{\gamma}_{(2 j+1)(n-1)}+\Delta_{(2 j+1) \delta_{2 j},(2 j+2)}+k_{2 j}-\sum_{\substack{((2 j+1) b) \in \mathcal{V}_{\mathrm{OPE}} \\
b \neq n-1}} \gamma_{(2 j+1) b}\right)\right) \\
& \times\left(\prod _ { j = 1 } ^ { \frac { n } { 2 } - 3 } \Gamma \left(\tilde{\gamma}_{(2 j+2)(n-1)}+\Delta_{(2 j+2) \delta_{2 j+1},(2 j+1) \delta_{2 j-1}}+k_{2 j+1,2 j-1}+\sum_{\substack{(a(2 j+1)) \in \mathcal{V}_{\mathrm{OPE}} \\
a<2 j+1}} \gamma_{a(2 j+1)}\right.\right. \\
& \left.\left.-\sum_{\substack{((2 j+2) b) \in \mathcal{V}_{\mathrm{OPE}} \\
b>2 j+2, b \neq n-1}} \gamma_{(2 j+2) b}\right)\right) \Gamma\left(\tilde{\gamma}_{2(n-1)}+\Delta_{2 \delta_{n-3}, 1 \delta_{2} \delta_{4} \ldots \delta_{n-4}}+k_{(n-3), 24 \ldots(n-4)}+\sum_{j=3}^{n-2} \gamma_{1 j}+\sum_{\substack{3 \leq i<j \leq n-2 \\
(i j) \in \mathcal{V}_{\mathrm{OPE}}}} \gamma_{i j}\right) \\
& \times \Gamma\left(\Delta_{(n-2) \delta_{n-3},(n-3) \delta_{n-5}}+k_{n-3, n-5}+\sum_{j=1}^{n-4} \gamma_{j(n-3)}\right) .
\end{aligned}
$$

Now, we will integrate over $\gamma_{2(n-3)}, \gamma_{1(n-2)}, \gamma_{3(n-2)}, \gamma_{4(n-2)}, \ldots, \gamma_{(n-4)(n-2)}$. The dependence on all these variables has been packaged into $L_{n-2}$ above. We first integrate over $\gamma_{2(n-3)}$ using the first Barnes lemma (4.15), and then do a change of variables 
$\gamma_{i(n-3)} \rightarrow \gamma_{i(n-3)}-\gamma_{i(n-2)}$, for $i=1,3,4, \ldots, n-4$. Doing this, we obtain,

$$
\begin{aligned}
& \int \frac{d \gamma_{2(n-3)}}{2 \pi i} L_{n-2} \\
& =\Gamma\left(\widetilde{\gamma}_{1(n-1)}+\Delta_{1 \delta_{1}, 2}+k_{1}-\sum_{j=3}^{n-3} \gamma_{1 j}\right)\left(\prod _ { j = 1 } ^ { \frac { n } { 2 } - 3 } \Gamma \left(\widetilde{\gamma}_{(2 j+1)(n-1)}+\Delta_{(2 j+1) \delta_{2 j},(2 j+2)}+k_{2 j}\right.\right. \\
& \left.-\sum_{\substack{((2 j+1) b) \in \mathcal{V}_{\mathrm{OPE}} \\
b \neq n-1, n-2}} \gamma_{(2 j+1) b}\right) \Gamma\left(\widetilde{\gamma}_{2(n-1)}+\Delta_{2 \delta_{n-3}, 1 \delta_{2} \delta_{4} \ldots \delta_{n-4}}+k_{(n-3), 24 \ldots(n-4)}+\sum_{j=3}^{n-3} \gamma_{1 j}+\sum_{\substack{3 \leq i<j \leq n-3 \\
(i j) \in \mathcal{V}_{\mathrm{OPE}}}} \gamma_{i j}\right) \\
& \times\left(\prod _ { j = 1 } ^ { \frac { n } { 2 } - 3 } \Gamma \left(\widetilde{\gamma}_{(2 j+2)(n-1)}+\Delta_{(2 j+2) \delta_{2 j+1},(2 j+1) \delta_{2 j-1}}+k_{2 j+1,2 j-1}+\sum_{\substack{(a(2 j+1)) \in \mathcal{V}_{\mathrm{OPE}} \\
a<2 j+1}} \gamma_{a(2 j+1)}\right.\right. \\
& \left.\left.-\sum_{\substack{((2 j+2) b) \in \mathcal{V}_{\mathrm{OPE}} \\
b>2 j+2, b \neq n-1, n-2}} \gamma_{(2 j+2) b}\right)\right) \Gamma\left(\widetilde{\gamma}_{(n-3)(n-1)}+\Delta_{\delta_{n-3} \delta_{n-4}, \delta_{n-5}}+k_{(n-3)(n-4),(n-5)}\right) \\
& \times \frac{\Gamma\left(\widetilde{\gamma}_{2(n-3)}+\Delta_{\delta_{n-5} \delta_{n-4}, \delta_{n-3}}+k_{(n-5)(n-4),(n-3)}-\sum_{a=1, a \neq 2}^{n-4} \gamma_{a(n-3)}\right)}{\Gamma\left(\widetilde{\gamma}_{2(n-3)}+\widetilde{\gamma}_{(n-3)(n-1)}+\Delta_{\delta_{n-4}}+2 k_{n-4}-\sum_{a=1, a \neq 2}^{n-4} \gamma_{a(n-3)}\right)} \widetilde{L}_{n-2},
\end{aligned}
$$

where $\widetilde{L}_{n-2}$ is defined to be

$$
\begin{aligned}
\widetilde{L}_{n-2}:= & \left(\prod_{r=1, r \neq 2}^{n-4} \Gamma\left(\widetilde{\gamma}_{r(n-2)}+\gamma_{r(n-2)}\right)\right)\left(\prod_{r=1, r \neq 2}^{n-4} \Gamma\left(\widetilde{\gamma}_{r(n-3)}+\gamma_{r(n-3)}-\gamma_{r(n-2)}\right)\right) \\
& \times \Gamma\left(\widetilde{\gamma}_{2(n-3)}+\widetilde{\gamma}_{(n-3)(n-1)}+\Delta_{(n-3) \delta_{n-4},(n-2)}+k_{n-4}-\sum_{b=1, b \neq 2}^{n-4}\left(\gamma_{b(n-3)}-\gamma_{b(n-2)}\right)\right) \\
& \times \Gamma\left(\Delta_{(n-2) \delta_{n-4},(n-3)}+k_{n-4}-\sum_{j=1, j \neq 2}^{n-4} \gamma_{j(n-2)}\right)
\end{aligned}
$$

$\widetilde{L}_{n-2}$ collects the Mellin variable-dependent integrand for the integrals over $\gamma_{i(n-2)}$ for $i=1,3,4, \ldots, n-4$. Using (4.35) we can easily evaluate these integrals in one go to obtain

$$
\begin{aligned}
& \left(\prod_{r=1, r \neq 2}^{n-4} \int \frac{d \gamma_{r(n-2)}}{2 \pi i}\right) M_{n-2} \\
& =\left(\prod_{i=1, i \neq 2}^{n-4} \Gamma\left(\widetilde{\gamma}_{i(n-2)}+\widetilde{\gamma}_{i(n-3)}+\gamma_{i(n-3)}\right)\right) \Gamma\left(\widetilde{\gamma}_{2(n-3)}+\widetilde{\gamma}_{(n-3)(n-1)}+\Delta_{\delta_{n-4}}+2 k_{n-4}\right. \\
& \left.\quad-\sum_{a=1, a \neq 2}^{n-4} \gamma_{a(n-3)}\right) \frac{\Gamma\left(\sum_{i=1, i \neq 2}^{n-4} \widetilde{\gamma}_{i(n-2)}+\Delta_{(n-2) \delta_{n-4},(n-3)}+k_{n-4}\right)}{\Gamma\left(\sum_{i=1}^{n-4} \widetilde{\gamma}_{i(n-3)}+\widetilde{\gamma}_{(n-3)(n-1)}+\sum_{i=1, i \neq 2}^{n-4} \widetilde{\gamma}_{i(n-2)}+\Delta_{\delta_{n-4}}+2 k_{n-4}\right)} \\
& \quad \times \Gamma\left(\sum_{i=1, i \neq 2}^{n-4} \widetilde{\gamma}_{i(n-3)}+\widetilde{\gamma}_{2(n-3)}+\widetilde{\gamma}_{(n-3)(n-1)}+\Delta_{(n-3) \delta_{n-4},(n-2)}+k_{n-4}\right) .
\end{aligned}
$$


Substituting these results back in $I$, we obtain

$$
\begin{aligned}
& I=\Gamma\left(\Delta_{12, \delta_{1}}-k_{1}\right)\left(\prod_{j=1}^{\frac{n}{2}-3} \Gamma\left(\Delta_{(2 j+1)(2 j+2), \delta_{2 j}}-k_{2 j}\right)\right)\left(\prod_{(r s) \in \mathcal{V}_{\mathrm{OPE}}} \int \frac{d \widetilde{\gamma}_{r s}}{2 \pi i} \Gamma\left(-\widetilde{\gamma}_{r s}\right)\left(v_{r s}-1\right)^{\widetilde{\gamma}_{r s}}\right) \\
& \times\left(\prod_{\substack{(r s) \in \mathcal{V}_{\mathrm{OPE}} \\
s \neq n-1, n-2, n-3}} \int \frac{d \gamma_{r s}}{2 \pi i} \Gamma\left(\widetilde{\gamma}_{r s}+\gamma_{r s}\right)\right)\left(\prod_{r=1, r \neq 2}^{n-4} \int \frac{d \gamma_{r(n-3)}}{2 \pi i} \Gamma\left(\sum_{b=n-3}^{n-2} \widetilde{\gamma}_{r b}+\gamma_{r(n-3)}\right)\right) \\
& \times W_{n-3} W_{n-4}\left(\prod_{j=1}^{\frac{n}{2}-3} \Gamma\left(\Delta_{\delta_{2 j-1} \delta_{2 j}, \delta_{2 j+1}}+k_{(2 j-1)(2 j),(2 j+1)}-\sum_{\substack{(a b) \in \mathcal{V}_{\mathrm{OPE}} \\
a<b, b=2 j+1 \text { or } 2 j+2}} \gamma_{a b}\right)\right) \\
& \times \Gamma\left(\widetilde{\gamma}_{2(n-3)}+\Delta_{\delta_{n-5} \delta_{n-4}, \delta_{n-3}}+k_{(n-5)(n-4),(n-3)}-\sum_{a=1, a \neq 2}^{n-4} \gamma_{a(n-3)}\right) \\
& \times \Gamma\left(\widetilde{\gamma}_{(n-3)(n-1)}+\Delta_{\delta_{n-3} \delta_{n-4}, \delta_{n-5}}+k_{(n-3)(n-4),(n-5)}\right) \Gamma\left(\widetilde{\gamma}_{1(n-1)}+\Delta_{1 \delta_{1}, 2}+k_{1}-\sum_{j=3}^{n-3} \gamma_{1 j}\right) \\
& \times \Gamma\left(\widetilde{\gamma}_{2(n-1)}+\Delta_{2 \delta_{n-3}, 1 \delta_{2} \delta_{4} \ldots \delta_{n-4}}+k_{(n-3), 24 \ldots(n-4)}+\sum_{j=3}^{n-3} \gamma_{1 j}+\sum_{\substack{3 \leq i<j \leq n-3 \\
(i j) \in \mathcal{V}_{\mathrm{OPE}}}} \gamma_{i j}\right) \\
& \times\left(\prod_{j=1}^{\frac{n}{2}-3} \Gamma\left(\widetilde{\gamma}_{(2 j+1)(n-1)}+\Delta_{(2 j+1) \delta_{2 j},(2 j+2)}+k_{2 j}-\sum_{\substack{((2 j+1) b) \in \mathcal{V}_{\mathrm{OPE}} \\
b \neq n-1, n-2}} \gamma_{(2 j+1) b}\right)\right) \\
& \times\left(\prod _ { j = 1 } ^ { \frac { n } { 2 } - 3 } \Gamma \left(\widetilde{\gamma}_{(2 j+2)(n-1)}+\Delta_{(2 j+2) \delta_{2 j+1},(2 j+1) \delta_{2 j-1}}+k_{2 j+1,2 j-1}+\sum_{\substack{(a(2 j+1)) \in \mathcal{V}_{\mathrm{OPE}} \\
a<2 j+1}} \gamma_{a(2 j+1)}\right.\right. \\
& \left.\left.-\sum_{\substack{((2 j+2) b) \in \mathcal{V}_{\mathrm{OPE}} \\
b>2 j+2, b \neq n-1, n-2}} \gamma_{(2 j+2) b}\right)\right)
\end{aligned}
$$

where we have defined

$$
\begin{aligned}
W_{n-4}:= & \frac{\Gamma\left(\Delta_{(n-3)(n-2), \delta_{n-4}}-k_{n-4}\right) \Gamma\left(\sum_{(i(n-2)) \in \mathcal{V}_{\mathrm{OPE}}} \tilde{\gamma}_{i(n-2)}+\Delta_{(n-2) \delta_{n-4},(n-3)}+k_{n-4}\right)}{\Gamma\left(\sum_{(i(n-3)) \in \mathcal{V}_{\mathrm{OPE}}} \widetilde{\gamma}_{i(n-3)}+\sum_{(i(n-2)) \in \mathcal{V}_{\mathrm{OPE}}} \widetilde{\gamma}_{i(n-2)}+\Delta_{\delta_{n-4}}+2 k_{n-4}\right)} \\
& \times \Gamma\left(\sum_{(i(n-3)) \in \mathcal{V}_{\mathrm{OPE}}} \widetilde{\gamma}_{i(n-3)}+\Delta_{(n-3) \delta_{n-4},(n-2)}+k_{n-4}\right) .
\end{aligned}
$$




\section{B.3.2 Integrals over a green-colored chain}

First, integrating $L_{n-2 K-1}$, defined in (4.60), over $\gamma_{1(n-2 K-1)}$ we get

$$
\begin{aligned}
& \int \frac{d \gamma_{1(n-2 K-1)}}{2 \pi i} L_{n-2 K-1} \\
& =\Gamma\left(\widetilde{\gamma}_{1(n-2 K)}+\widetilde{\gamma}_{1(n-2 K-1)}+\sum_{j=n-2 K+1}^{n-1} \widetilde{\gamma}_{1 j}+\Delta_{1 \delta_{1}, 2}+k_{1}-\sum_{j=3}^{n-2 K-2} \gamma_{1 j}\right) \\
& \times \Gamma\left(\sum_{i=0}^{K-1} \widetilde{\gamma}_{2(n-2 i-1)}+\widetilde{\gamma}_{2(n-2 K-1)}+\Delta_{2 \delta_{n-2 K-3}, 1 \delta_{2} \delta_{4} \ldots \delta_{n-2 K-4}}+k_{(n-2 K-3), 24 \ldots(n-2 K-4)}\right. \\
& \left.\quad+\sum_{j=3}^{n-2 K-2} \gamma_{1 j}+\sum_{3 \leq i<j \leq n-2 K-2} \gamma_{i j}\right) \\
& \times \Gamma\left(\sum_{i=0}^{K} \widetilde{\gamma}_{2(n-2 i-1)}+\sum_{j=n-2 K-1}^{n-1} \widetilde{\gamma}_{1 j}+\Delta_{\delta_{n-2 K-3} \delta_{1}, \delta_{2} \delta_{4} \ldots \delta_{n-2 K-4}}+k_{(n-2 K-3) 1,24 \ldots(n-2 K-4)}\right. \\
& \left.\quad+\sum_{3 \leq i<j \leq n-2 K-2} \gamma_{i j}\right)_{(i j) \in \mathcal{V}_{\mathrm{OPE}}}^{-1} \widetilde{L}_{n-2 K-1}
\end{aligned}
$$

where

$$
\begin{aligned}
\widetilde{L}_{n-2 K-1}:= & \left(\prod_{r=3}^{n-2 K-2} \Gamma\left(\widetilde{\gamma}_{r(n-2 K)}+\widetilde{\gamma}_{r(n-2 K-1)}+\gamma_{r(n-2 K-1)}\right)\right) \\
\times & \left(\prod_{j=1}^{\frac{n}{2}-K-2} \Gamma\left(\sum_{a=n-2 K+1}^{n-1} \widetilde{\gamma}_{(2 j+1) a}+\Delta_{(2 j+1) \delta_{2 j},(2 j+2)}+k_{2 j}-\sum_{\substack{((2 j+1) b) \in \mathcal{V}_{\mathrm{OPE}} \\
b \neq n-2 K, \ldots, n-1}} \gamma_{(2 j+1) b}\right)\right) \\
\times & \left(\prod _ { j = 1 } ^ { \frac { n } { 2 } - K - 2 } \Gamma \left(\sum_{a=n-2 K+1}^{n-1} \widetilde{\gamma}_{(2 j+2) a}+\Delta_{(2 j+2) \delta_{2 j+1},(2 j+1) \delta_{2 j-1}}+k_{2 j+1,2 j-1}\right.\right. \\
& \left.\left.+\sum_{\substack{(b(2 j+1)) \in \mathcal{V}_{\mathrm{OPE}} \\
b<2 j+1}} \gamma_{b(2 j+1)}-\sum_{\substack{((2 j+2) b) \in \mathcal{V}_{\mathrm{OPE}} \\
b>2 j+2, b \neq n-2 K, \ldots, n-1}} \gamma_{(2 j+2) b}\right)\right) \\
\times & \left(\sum_{i=0}^{K-1} \widetilde{\gamma}_{2(n-2 i-1)}+\sum_{j=n-2 K+1}^{n-1} \widetilde{\gamma}_{1 j}+\Delta_{\delta_{n-2 K-1} \delta_{1}, \delta_{2} \delta_{4} \ldots \delta_{n-2 K-2}}+k_{(n-2 K-1) 1,24 \ldots(n-2 K-2)}\right. \\
& \left.+\sum_{3 \leq i<j \leq n-2 K-1} \gamma_{i j}\right) \\
& \times \Gamma\left(\widetilde{\gamma}_{1(n-2 K) \in \mathcal{V}_{\mathrm{OPE}}}+\widetilde{\gamma}_{1(n-2 K-1)}+\widetilde{\gamma}_{2(n-2 K-1)}+\Delta_{\delta_{n-2} K-3} \delta_{n-2 K-2}, \delta_{n-2 K-1}\right. \\
& \left.+k_{(n-2 K-3)(n-2 K-2),(n-2 K-1)}-\sum_{a=3}^{n-2 K-2} \gamma_{a(n-2 K-1)}\right) .
\end{aligned}
$$


Next, we will integrate $\widetilde{L}_{n-2 K-1}$ over the Mellin variables $\gamma_{3(n-2 K-1)}, \gamma_{4(n-2 K-1)}, \ldots, \gamma_{(n-2 K-2)(n-2 K-1)}$ using the inductive Barnes lemma (4.35). The integrand $\widetilde{L}_{n-2 K-1}$ matches the integrand of (4.35) with the coefficient assignments:

$$
\begin{aligned}
& A_{r}=\widetilde{\gamma}_{(r+2)(n-2 K)}+\widetilde{\gamma}_{(r+2)(n-2 K-1)} \\
& \int \sum_{a=n-2 K+1}^{n-1} \widetilde{\gamma}_{(r+2) a}+\Delta_{(r+2) \delta_{r+1},(r+3)}+k_{r+1}-\sum_{\substack{((r+2) b) \in \mathcal{V}_{\mathrm{OPE}} \\
b \neq n-2 K-1, \ldots, n-1}} \gamma_{(r+2) b} \quad r \text { odd } \\
& B_{r}=\left\{\sum_{a=n-2 K+1}^{n-1} \tilde{\gamma}_{(r+2) a}+\Delta_{(r+2) \delta_{r+1},(r+1) \delta_{r-1}}+k_{r+1, r-1}+\sum_{\substack{(b(r+1)) \in \mathcal{V}_{\mathrm{OPE}} \\
b<r+1}} \gamma_{b(r+1)}\right. \\
& \text { - } \quad \sum \gamma_{(r+2) b} \quad r \text { even } \\
& \begin{array}{c}
((r+2) b) \in \mathcal{V}_{\mathrm{OPE}} \\
b>r+2, b \neq n-2 K-1, \ldots, n-1
\end{array} \\
& C=\sum_{i=0}^{K-1} \widetilde{\gamma}_{2(n-2 i-1)}+\sum_{j=n-2 K+1}^{n-1} \widetilde{\gamma}_{1 j}+\Delta_{\delta_{n-2 K-1} \delta_{1}, \delta_{2} \delta_{4} \ldots \delta_{n-2 K-2}}+k_{(n-2 K-1) 1,24 \ldots(n-2 K-2)} \\
& +\sum_{3 \leq i<j \leq n-2 K-2} \gamma_{i j} \\
& (i j) \in \mathcal{V}_{\mathrm{OPE}} \\
& D=\widetilde{\gamma}_{1(n-2 K)}+\widetilde{\gamma}_{1(n-2 K-1)}+\widetilde{\gamma}_{2(n-2 K-1)}+\Delta_{\delta_{n-2 K-3} \delta_{n-2 K-2}, \delta_{n-2 K-1}} \\
& +k_{(n-2 K-3)(n-2 K-2),(n-2 K-1)} \text {, }
\end{aligned}
$$

for $1 \leq r \leq n-2 K-4$. Then applying (4.35), we obtain

$$
\begin{aligned}
& \left(\prod_{r=1}^{n-2 K-4} \int \frac{d \gamma_{(r+2)(n-2 K-1)}}{2 \pi i}\right) \widetilde{L}_{n-2 K-1} \\
& =\frac{\Gamma\left(\sum_{i=0}^{K} \widetilde{\gamma}_{2(n-2 i-1)}+\sum_{j=n-2 K-1}^{n-1} \widetilde{\gamma}_{1 j}+\Delta_{\delta_{n-2 K-3} \delta_{1}, \delta_{2} \delta_{4} \ldots \delta_{n-2 K-4}}\right.}{\Gamma\left(\sum_{i=1, i \neq 2}^{n-2 K-2} \sum_{j=n-2 K-1}^{n-1} \widetilde{\gamma}_{i j}+\sum_{i=0}^{K} \widetilde{\gamma}_{2(n-2 i-1)}+\Delta_{\delta_{n-2 K-3}}+2 k_{n-2 K-3}\right)} \\
& \left.+k_{(n-2 K-3) 1,24 \ldots(n-2 K-4)}+\sum_{\substack{3 \leq i<j \leq n-2 K-2 \\
(i j) \in \mathcal{V}_{\mathrm{OPE}}}} \gamma_{i j}\right) \\
& \overline{\Gamma\left(\sum_{i=1, i \neq 2}^{n-2 K-2} \sum_{j=n-2 K-1}^{n-1} \widetilde{\gamma}_{i j}+\sum_{i=0}^{K} \widetilde{\gamma}_{2(n-2 i-1)}+\Delta_{\delta_{n-2 K-3}}+2 k_{n-2 K-3}\right)} \\
& \times\left(\prod_{j=1}^{\frac{n}{2}-K-2} \Gamma\left(\sum_{a=n-2 K-1}^{n-1} \widetilde{\gamma}_{(2 j+1) a}+\Delta_{(2 j+1) \delta_{2 j},(2 j+2)}+k_{2 j}-\sum_{\substack{((2 j+1) b) \in \mathcal{V}_{\mathrm{OPE}} \\
b \neq n-2 K-1, \ldots, n-1}} \gamma_{(2 j+1) b}\right)\right) \\
& \times\left(\prod _ { j = 1 } ^ { \frac { n } { 2 } - K - 2 } \Gamma \left(\sum_{a=n-2 K-1}^{n-1} \widetilde{\gamma}_{(2 j+2) a}+\Delta_{(2 j+2) \delta_{2 j+1},(2 j+1) \delta_{2 j-1}}+k_{2 j+1,2 j-1}\right.\right. \\
& \left.+\sum_{\substack{(b(2 j+1)) \in \mathcal{V}_{\mathrm{OPE}} \\
b<2 j+1}} \gamma_{b(2 j+1)}-\sum_{\substack{((2 j+2) b) \in \mathcal{V}_{\mathrm{OPE}} \\
b>2 j+2, b \neq n-2 K-1, \ldots, n-1}} \gamma_{(2 j+2) b}\right)
\end{aligned}
$$




$$
\begin{aligned}
& \times \Gamma\left(\sum_{i=1, i \neq 2}^{n-2 K-2} \widetilde{\gamma}_{i(n-2 K)}+\sum_{i=1}^{n-2 K-2} \widetilde{\gamma}_{i(n-2 K-1)}\right. \\
+ & \left.\Delta_{\delta_{n-2 K-3} \delta_{n-2 K-2}, \delta_{n-2 K-1}}+k_{(n-2 K-3)(n-2 K-2),(n-2 K-1)}\right) \\
\times & \Gamma\left(\sum_{i=1, i \neq 2}^{n-2 K-2} \sum_{j=n-2 K+1}^{n-1} \widetilde{\gamma}_{i j}+\sum_{i=0}^{K-1} \widetilde{\gamma}_{2(n-2 i-1)}\right. \\
& \left.+\Delta_{\delta_{n-2 K-3} \delta_{n-2 K-1}, \delta_{n-2 K-2}}+k_{(n-2 K-3)(n-2 K-1),(n-2 K-2)}\right)
\end{aligned}
$$

where we used

$$
\begin{aligned}
& \sum_{r=1}^{n-2 K-4} B_{r}=\sum_{i=3}^{n-2 K-2} \sum_{j=n-2 K+1}^{n-1} \widetilde{\gamma}_{i j}+\Delta_{\delta_{2} \delta_{4} \delta_{6} \ldots \delta_{n-2 K-4} \delta_{n-2 K-3}, \delta_{1}}+k_{246 \ldots(n-2 K-4)(n-2 K-3), 1} \\
& -\sum_{\substack{3 \leq a<b \leq n-2 K-2 \\
(a b) \in \mathcal{V}_{\mathrm{OPE}}}} \gamma_{a b}
\end{aligned}
$$

Substituting the results of these integrations back into $\widehat{I}_{n-2 K-1}$ in (4.56), we get

$$
\begin{aligned}
& \widehat{I}_{n-2 K-1}=\Gamma\left(\Delta_{12, \delta_{1}}-k_{1}\right)\left(\prod_{j=1}^{\frac{n}{2}-K-2} \Gamma\left(\Delta_{(2 j+1)(2 j+2), \delta_{2 j}}-k_{2 j}\right)\right)\left(\prod_{(r s) \in \mathcal{V}_{\mathrm{OPE}}} \int \frac{d \widetilde{\gamma}_{r s}}{2 \pi i} \Gamma\left(-\widetilde{\gamma}_{r s}\right)\left(v_{r s}-1\right)^{\widetilde{\gamma}_{r s}}\right) \\
& \times\left(\prod_{j=n-2 K-3}^{n-3} W_{j}\right)\left(\prod_{\substack{(r s) \in \mathcal{V}_{\mathrm{OPE}} \\
s \neq n-2 K-1, \ldots, n-1}} \int \frac{d \gamma_{r s}}{2 \pi i} \Gamma\left(\widetilde{\gamma}_{r s}+\gamma_{r s}\right)\right) \\
& \times\left(\prod_{j=1}^{\frac{n}{2}-K-2} \Gamma\left(\Delta_{\delta_{2 j-1} \delta_{2 j}, \delta_{2 j+1}}+k_{(2 j-1)(2 j),(2 j+1)}-\sum_{\substack{(a b) \in \mathcal{V}_{\mathrm{OPE}} \\
a<b, b=2 j+1}} \gamma_{a b}\right)\right) \\
& \times \Gamma\left(\widetilde{\gamma}_{1(n-2 K)}+\widetilde{\gamma}_{1(n-2 K-1)}+\sum_{j=n-2 K+1}^{n-1} \widetilde{\gamma}_{1 j}+\Delta_{1 \delta_{1}, 2}+k_{1}-\sum_{j=3}^{n-2 K-2} \gamma_{1 j}\right) \\
& \times \Gamma\left(\sum_{i=0}^{K-1} \widetilde{\gamma}_{2(n-2 i-1)}+\widetilde{\gamma}_{2(n-2 K-1)}+\Delta_{2 \delta_{n-2 K-3}, 1 \delta_{2} \delta_{4} \ldots \delta_{n-2 K-4}}+k_{(n-2 K-3), 24 \ldots(n-2 K-4)}\right. \\
& \left.+\sum_{j=3}^{n-2 K-2} \gamma_{1 j}+\sum_{\substack{3 \leq i<j \leq n-2 K-2 \\
(i j) \in \mathcal{V}_{\mathrm{OPE}}}} \gamma_{i j}\right) \\
& \times\left(\prod_{j=1}^{\frac{n}{2}-K-2} \Gamma\left(\sum_{a=n-2 K-1}^{n-1} \widetilde{\gamma}_{(2 j+1) a}+\Delta_{(2 j+1) \delta_{2 j},(2 j+2)}+k_{2 j}-\sum_{\substack{((2 j+1) b) \in \mathcal{V}_{\mathrm{OPE}} \\
b \neq n-2 K-1, \ldots, n-1}} \gamma_{(2 j+1) b}\right)\right) \\
& \times\left(\prod _ { j = 1 } ^ { \frac { n } { 2 } - K - 2 } \Gamma \left(\sum_{a=n-2 K-1}^{n-1} \widetilde{\gamma}_{(2 j+2) a}+\Delta_{(2 j+2) \delta_{2 j+1},(2 j+1) \delta_{2 j-1}}+k_{2 j+1,2 j-1}\right.\right. \\
& \left.+\sum_{\substack{(b(2 j+1)) \in \mathcal{V}_{\mathrm{OPE}} \\
b<2 j+1}} \gamma_{b(2 j+1)}-\sum_{\substack{((2 j+2) b) \in \mathcal{V}_{\mathrm{OPE}} \\
b>2 j+2, b \neq n-2 K-1, \ldots, n-1}} \gamma_{(2 j+2) b}\right),
\end{aligned}
$$


where

$$
\begin{aligned}
& W_{n-2 K-3} \\
& :=\Gamma\left(\sum_{i=1, i \neq 2}^{n-2 K-2} \widetilde{\gamma}_{i(n-2 K)}+\sum_{i=1}^{n-2 K-2} \tilde{\gamma}_{i(n-2 K-1)}+\Delta_{\delta_{n-2 K-3} \delta_{n-2 K-2}, \delta_{n-2 K-1}}+k_{(n-2 K-3)(n-2 K-2),(n-2 K-1)}\right) \\
& \times \Gamma\left(\sum_{i=1, i \neq 2}^{n-2 K-2} \sum_{j=n-2 K+1}^{n-1} \tilde{\gamma}_{i j}+\sum_{i=0}^{K-1} \tilde{\gamma}_{2(n-2 i-1)}+\Delta_{\delta_{n-2 K-3} \delta_{n-2 K-1}, \delta_{n-2 K-2}}+k_{(n-2 K-3)(n-2 K-1),(n-2 K-2)}\right) \\
& \times \frac{\Gamma\left(\sum_{a=n-2 K+1}^{n-1} \tilde{\gamma}_{(n-2 K) a}+\sum_{a=n-2 K+1}^{n-1} \tilde{\gamma}_{(n-2 K-1) a}+\Delta_{\delta_{n-2 K-1} \delta_{n-2 K-2}, \delta_{n-2 K-3}}+k_{(n-2 K-1)(n-2 K-2),(n-2 K-3)}\right)}{\Gamma\left(\sum_{i=1, i \neq 2}^{n-2 K-2} \sum_{j=n-2 K-1}^{n-1} \widetilde{\gamma}_{i j}+\sum_{i=0}^{K} \widetilde{\gamma}_{2(n-2 i-1)}+\Delta_{\delta_{n-2 K-3}}+2 k_{n-2 K-3}\right)},
\end{aligned}
$$

which agrees with $W_{j}$ in (4.58) after setting $j=n-2 K-3$ (i.e. for odd $j$ ).

\section{B.3.3 Integrals over a magenta-colored chain}

Integrating (4.62) over $\gamma_{2(n-2 K-3)}$ using the first Barnes lemma (4.15), and shifting variables $\gamma_{i(n-2 K-3)} \rightarrow \gamma_{i(n-2 K-3)}-\gamma_{i(n-2 K-2)}$ for $i=1,3,4, \ldots, n-2 K-4$, we get

$$
\begin{aligned}
& \int \frac{d \gamma_{2(n-2 K-3)}}{2 \pi i} L_{n-2 K-2}=\left(\prod_{j=1}^{\frac{n}{2}-K-3} \Gamma\left(\Delta_{\delta_{2 j-1} \delta_{2 j}, \delta_{2 j+1}}+k_{(2 j-1)(2 j),(2 j+1)}-\sum_{\substack{(a b) \in \mathcal{V}_{\mathrm{OPE}} \\
a<b, b=2 j+1 \text { or } 2 j+2}} \gamma_{a b}\right)\right) \\
& \times \Gamma\left(\widetilde{\gamma}_{1(n-2 K)}+\widetilde{\gamma}_{1(n-2 K-1)}+\sum_{j=n-2 K+1}^{n-1} \widetilde{\gamma}_{1 j}+\Delta_{1 \delta_{1}, 2}+k_{1}-\sum_{j=3}^{n-2 K-3} \gamma_{1 j}\right) \\
& \times \Gamma\left(\sum_{i=0}^{K-1} \widetilde{\gamma}_{2(n-2 i-1)}+\widetilde{\gamma}_{2(n-2 K-1)}+\Delta_{2 \delta_{n-2 K-3}, 1 \delta_{2} \delta_{4} \ldots \delta_{n-2 K-4}}+k_{(n-2 K-3), 24 \ldots(n-2 K-4)}+\sum_{j=3}^{n-2 K-3} \gamma_{1 j}\right. \\
& \left.+\sum_{\substack{3 \leq i<j \leq n-2 K-3 \\
(i j) \in \mathcal{V}_{\mathrm{OPE}}}} \gamma_{i j}\right) \widetilde{L}_{n-2 K-2} \\
& \times\left(\prod_{j=1}^{\frac{n}{2}-K-3} \Gamma\left(\sum_{a=n-2 K-1}^{n-1} \widetilde{\gamma}_{(2 j+1) a}+\Delta_{(2 j+1) \delta_{2 j},(2 j+2)}+k_{2 j}-\sum_{\substack{((2 j+1) b) \in \mathcal{V}_{\mathrm{OPE}} \\
b \neq n-2 K-2, \ldots, n-1}} \gamma_{(2 j+1) b}\right)\right) \\
& \times\left(\prod _ { j = 1 } ^ { \frac { n } { 2 } - K - 3 } \Gamma \left(\sum_{a=n-2 K-1}^{n-1} \widetilde{\gamma}_{(2 j+2) a}+\Delta_{(2 j+2) \delta_{2 j+1},(2 j+1) \delta_{2 j-1}}+k_{2 j+1,2 j-1}+\sum_{\substack{(b(2 j+1)) \in \mathcal{V}_{\mathrm{OPE}} \\
b<2 j+1}} \gamma_{b(2 j+1)}\right.\right. \\
& \left.\left.-\sum_{\substack{((2 j+2) b) \in \mathcal{V}_{\mathrm{OPE}} \\
b>2 j+2, b \neq n-2 K-2, \ldots, n-1}} \gamma_{(2 j+2) b}\right)\right) \\
& \times \Gamma\left(\widetilde{\gamma}_{2(n-2 K-3)}+\Delta_{\delta_{n-2 K-5} \delta_{n-2 K-4}, \delta_{n-2 K-3}}+k_{(n-2 K-5)(n-2 K-4),(n-2 K-3)}-\sum_{a=1, a \neq 2}^{n-2 K-4} \gamma_{a(n-2 K-3)}\right) \\
& \times \Gamma \sum_{\substack{((n-2 K-2) a) \in \mathcal{V}_{\mathrm{OPE}} \\
a>n-2 K-2}} \tilde{\gamma}_{(n-2 K-2) a}+\sum_{\substack{((n-2 K-3) a) \in \mathcal{V}_{\mathrm{OPE}} \\
a>n-2 K-2}} \tilde{\gamma}_{(n-2 K-3) a}+\Delta_{\delta_{n-2 K-3} \delta_{n-2 K-4}, \delta_{n-2 K-5}} \\
& \left.+k_{(n-2 K-3)(n-2 K-4),(n-2 K-5)}\right)
\end{aligned}
$$




$$
\begin{aligned}
& \times \Gamma\left(\tilde{\gamma}_{2(n-2 K-3)}+\sum_{\substack{((n-2 K-2) a) \in \mathcal{V}_{\mathrm{OPE}} \\
a>n-2 K-2}} \widetilde{\gamma}_{(n-2 K-2) a}+\sum_{\substack{((n-2 K-3) a) \in \mathcal{V}_{\mathrm{OPE}} \\
a>n-2 K-2}} \tilde{\gamma}_{(n-2 K-3) a}+\Delta_{\delta_{n-2 K-4}}+2 k_{n-2 K-4}\right. \\
& \left.\quad-\sum_{a=1, a \neq 2}^{n-2 K-4} \gamma_{a(n-2 K-3)}\right)^{-1}
\end{aligned}
$$

where we have defined

$$
\begin{aligned}
& \widetilde{L}_{n-2 K-2} \\
& :=\left(\prod_{r=1, r \neq 2}^{n-2 K-4} \Gamma\left(\widetilde{\gamma}_{r(n-2 K-2)}+\gamma_{r(n-2 K-2)}\right)\right)\left(\prod_{r=1, r \neq 2}^{n-2 K-4} \Gamma\left(\widetilde{\gamma}_{r(n-2 K-3)}+\gamma_{r(n-2 K-3)}-\gamma_{r(n-2 K-2)}\right)\right) \\
& \times \Gamma\left(\widetilde{\gamma}_{2(n-2 K-3)}+\sum_{a=n-2 K-1}^{n-1} \widetilde{\gamma}_{(n-2 K-3) a}+\Delta_{(n-2 K-3) \delta_{n-2 K-4},(n-2 K-2)}+k_{n-2 K-4}\right. \\
& \left.\quad-\sum_{a=1, a \neq 2}^{n-2 K-4} \gamma_{a(n-2 K-3)}+\sum_{a=1, a \neq 2}^{n-2 K-4} \gamma_{a(n-2 K-2)}\right) \\
& \times \Gamma\left(\sum_{\substack{((n-2 K-2) a) \in \mathcal{V}_{\mathrm{OPE}} \\
a>n-2 K-2}}^{\widetilde{\gamma}_{(n-2 K-2) a}+\Delta_{(n-2 K-2) \delta_{n-2 K-4},(n-2 K-3)}+k_{n-2 K-4}-\sum_{a=1, a \neq 2}^{n-2 K-4} \gamma_{a(n-2 K-2)}}\right) .
\end{aligned}
$$

Now using (4.35), we can evaluate the integral $\left(\prod_{r=1, r \neq 2}^{n-2 K-4} \int \frac{d \gamma_{r(n-2 K-2)}}{2 \pi i}\right) \widetilde{L}_{n-2 K-2}$, to get

$$
\begin{aligned}
& \left(\prod_{r=1, r \neq 2}^{n-2 K-4} \int \frac{d \gamma_{r(n-2 K-2)}}{2 \pi i}\right) \widetilde{L}_{n-2 K-2} \\
& \left(\prod_{r=1, r \neq 2}^{n-2 K-4} \Gamma\left(\widetilde{\gamma}_{r(n-2 K-2)}+\widetilde{\gamma}_{r(n-2 K-3)}+\gamma_{r(n-2 K-3)}\right)\right)
\end{aligned}
$$

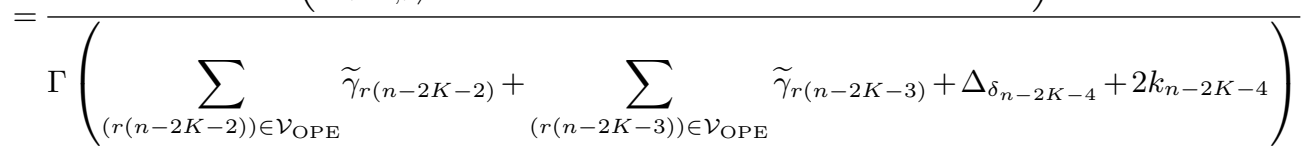

$$
\begin{aligned}
& \times \Gamma\left(\tilde{\gamma}_{2(n-2 K-3)}+\sum_{\substack{((n-2 K-2) a) \in \mathcal{V}_{\mathrm{OPE}} \\
a>n-2 K-2}} \tilde{\gamma}_{(n-2 K-2) a}+\sum_{\substack{((n-2 K-3) a) \in \mathcal{V}_{\mathrm{OPE}} \\
a>n-2 K-2}} \tilde{\gamma}_{(n-2 K-3) a}+\Delta_{\delta_{n-2 K-4}}+2 k_{n-2 K-4}\right. \\
& \left.-\sum_{a=1, a \neq 2}^{n-2 K-4} \gamma_{a(n-2 K-3)}\right) \Gamma\left(\sum_{(r(n-2 K-2)) \in \mathcal{V}_{\mathrm{OPE}}} \tilde{\gamma}_{r(n-2 K-2)}+\Delta_{(n-2 K-2) \delta_{n-2 K-4},(n-2 K-3)}+k_{n-2 K-4}\right) \\
& \times \Gamma\left(\sum_{(r(n-2 K-3)) \in \mathcal{V}_{\mathrm{OPE}}} \widetilde{\gamma}_{r(n-2 K-3)}+\Delta_{(n-2 K-3) \delta_{n-2 K-4},(n-2 K-2)}+k_{n-2 K-4}\right) .
\end{aligned}
$$


Putting the results of these integrations back into $\widehat{I}_{n-2 K-1}$ in (4.61), we get

$$
\begin{aligned}
& \widehat{I}_{n-2 K-1} \\
& =\Gamma\left(\Delta_{12, \delta_{1}}-k_{1}\right)\left(\prod_{j=1}^{\frac{n}{2}-K-3} \Gamma\left(\Delta_{(2 j+1)(2 j+2), \delta_{2 j}}-k_{2 j}\right)\right)\left(\prod_{(r s) \in \mathcal{V}_{\mathrm{OPE}}} \int \frac{d \widetilde{\gamma}_{r s}}{2 \pi i} \Gamma\left(-\widetilde{\gamma}_{r s}\right)\left(v_{r s}-1\right)^{\tilde{\gamma}_{r s}}\right) \\
& \times\left(\prod_{j=n-2 K-4}^{n-3} W_{j}\right)\left(\prod_{\substack{(r s) \in \mathcal{V}_{\mathrm{OPE}} \\
s \neq n-2 K-3, \ldots, n-1}} \int \frac{d \gamma_{r s}}{2 \pi i} \Gamma\left(\widetilde{\gamma}_{r s}+\gamma_{r s}\right)\right)\left(\prod_{r=1, r \neq 2}^{n-2 K-4} \int \frac{d \gamma_{r(n-2 K-3)}}{2 \pi i}\right) \\
& \times\left(\prod_{j=1}^{\frac{n}{2}-K-3} \Gamma\left(\Delta_{\delta_{2 j-1} \delta_{2 j}, \delta_{2 j+1}}+k_{(2 j-1)(2 j),(2 j+1)}-\sum_{\substack{(a b) \in \mathcal{V}_{\mathrm{OPE}} \\
a<b, b=2 j+1 \text { or } 2 j+2}} \gamma_{a b}\right)\right) \\
& \times \Gamma\left(\widetilde{\gamma}_{1(n-2 K)}+\widetilde{\gamma}_{1(n-2 K-1)}+\sum_{j=n-2 K+1}^{n-1} \widetilde{\gamma}_{1 j}+\Delta_{1 \delta_{1}, 2}+k_{1}-\sum_{j=3}^{n-2 K-3} \gamma_{1 j}\right) \\
& \times \Gamma\left(\sum_{i=0}^{K-1} \widetilde{\gamma}_{2(n-2 i-1)}+\widetilde{\gamma}_{2(n-2 K-1)}+\Delta_{2 \delta_{n-2 K-3}, 1 \delta_{2} \delta_{4} \ldots \delta_{n-2 K-4}}+k_{(n-2 K-3), 24 \ldots(n-2 K-4)}\right. \\
& \left.+\sum_{j=3}^{n-2 K-3} \gamma_{1 j}+\sum_{\substack{3 \leq i<j \leq n-2 K-3 \\
(i j) \in \mathcal{V}_{\mathrm{OPE}}}} \gamma_{i j}\right) \\
& \times\left(\prod_{j=1}^{\frac{n}{2}-K-3} \Gamma\left(\sum_{a=n-2 K-1}^{n-1} \widetilde{\gamma}_{(2 j+1) a}+\Delta_{(2 j+1) \delta_{2 j},(2 j+2)}+k_{2 j}-\sum_{\substack{((2 j+1) b) \in \mathcal{V}_{\mathrm{OPE}} \\
b \neq n-2 K-2, \ldots, n-1}} \gamma_{(2 j+1) b}\right)\right) \\
& \times\left(\prod _ { j = 1 } ^ { \frac { n } { 2 } - K - 3 } \Gamma \left(\sum_{a=n-2 K-1}^{n-1} \widetilde{\gamma}_{(2 j+2) a}+\Delta_{(2 j+2) \delta_{2 j+1},(2 j+1) \delta_{2 j-1}}+k_{2 j+1,2 j-1}+\sum_{\substack{(b(2 j+1)) \in \mathcal{V}_{\mathrm{OPE}} \\
b<2 j+1}} \gamma_{b(2 j+1)}\right.\right. \\
& \left.\left.-\sum_{\substack{((2 j+2) b) \in \mathcal{V}_{\mathrm{OPE}} \\
b>2 j+2, b \neq n-2 K-2, \ldots, n-1}} \gamma_{(2 j+2) b}\right)\right) \\
& \times \Gamma\left(\widetilde{\gamma}_{2(n-2 K-3)}+\Delta_{\delta_{n-2 K-5} \delta_{n-2 K-4}, \delta_{n-2 K-3}}+k_{(n-2 K-5)(n-2 K-4),(n-2 K-3)}-\sum_{a=1, a \neq 2}^{n-2 K-4} \gamma_{a(n-2 K-3)}\right) \\
& \times \Gamma \sum_{\substack{((n-2 K-2) a) \in \mathcal{V}_{\mathrm{OPE}} \\
a>n-2 K-2}} \tilde{\gamma}_{(n-2 K-2) a}+\sum_{\substack{((n-2 K-3) a) \in \mathcal{V}_{\mathrm{OPE}} \\
a>n-2 K-2}} \widetilde{\gamma}_{(n-2 K-3) a}+\Delta_{\delta_{n-2 K-3} \delta_{n-2 K-4}, \delta_{n-2 K-5}} \\
& \left.+k_{(n-2 K-3)(n-2 K-4),(n-2 K-5)}\right)\left(\prod_{r=1, r \neq 2}^{n-2 K-4} \Gamma\left(\widetilde{\gamma}_{r(n-2 K-2)}+\widetilde{\gamma}_{r(n-2 K-3)}+\gamma_{r(n-2 K-3)}\right)\right),
\end{aligned}
$$


where

$$
\begin{aligned}
W_{n-2 K-4} & \frac{\Gamma\left(\Delta_{(n-2 K-3)(n-2 K-2), \delta_{n-2 K-4}}-k_{n-2 K-4}\right)}{\Gamma\left(\sum_{(r(n-2 K-2)) \in \mathcal{V}_{\mathrm{OPE}}} \widetilde{\gamma}_{r(n-2 K-2)}+\sum_{(r(n-2 K-3)) \in \mathcal{V}_{\mathrm{OPE}}} \widetilde{\gamma}_{r(n-2 K-3)}+\Delta_{\delta_{n-2 K-4}}+2 k_{n-2 K-4}\right)} \\
& \times \Gamma\left(\sum_{(r(n-2 K-2)) \in \mathcal{V}_{\mathrm{OPE}}} \widetilde{\gamma}_{r(n-2 K-2)}+\Delta_{(n-2 K-2) \delta_{n-2 K-4},(n-2 K-3)}+k_{n-2 K-4}\right) \\
& \times \Gamma\left(\sum_{(r(n-2 K-3)) \in \mathcal{V}_{\mathrm{OPE}}} \tilde{\gamma}_{r(n-2 K-3)}+\Delta_{(n-2 K-3) \delta_{n-2 K-4},(n-2 K-2)}+k_{n-2 K-4}\right),
\end{aligned}
$$

which agrees with $W_{j}$ in (4.58) if we set $j=n-2 K-4$ (i.e. for even $j$ ).

Open Access. This article is distributed under the terms of the Creative Commons Attribution License (CC-BY 4.0), which permits any use, distribution and reproduction in any medium, provided the original author(s) and source are credited.

\section{References}

[1] S. Ferrara, A.F. Grillo and R. Gatto, Tensor representations of conformal algebra and conformally covariant operator product expansion, Annals Phys. 76 (1973) 161 [INSPIRE].

[2] A.M. Polyakov, Nonhamiltonian approach to conformal quantum field theory, Zh. Eksp. Teor. Fiz. 66 (1974) 23 [Sov. Phys. JETP 39 (1974) 9] [INSPIRE].

[3] R. Rattazzi, V.S. Rychkov, E. Tonni and A. Vichi, Bounding scalar operator dimensions in 4D CFT, JHEP 12 (2008) 031 [arXiv:0807.0004] [INSPIRE].

[4] D. Poland, S. Rychkov and A. Vichi, The conformal bootstrap: theory, numerical techniques, and applications, Rev. Mod. Phys. 91 (2019) 015002 [arXiv: 1805.04405] [INSPIRE].

[5] S. Ferrara, A.F. Grillo and R. Gatto, Manifestly conformal covariant operator-product expansion, Lett. Nuovo Cim. 2 (1971) 1363 [INSPIRE].

[6] S. Ferrara, A.F. Grillo, G. Parisi and R. Gatto, Covariant expansion of the conformal four-point function, Nucl. Phys. B 49 (1972) 77 [Erratum ibid. 53 (1973) 643] [InSPIRE].

[7] S. Ferrara, R. Gatto and A.F. Grillo, Properties of partial wave amplitudes in conformal invariant field theories, Nuovo Cim. A 26 (1975) 226 [INSPIRE].

[8] F.A. Dolan and H. Osborn, Conformal four point functions and the operator product expansion, Nucl. Phys. B 599 (2001) 459 [hep-th/0011040] [INSPIRE].

[9] F.A. Dolan and H. Osborn, Conformal partial waves and the operator product expansion, Nucl. Phys. B 678 (2004) 491 [hep-th/0309180] [INSPIRE].

[10] F.A. Dolan and H. Osborn, Conformal partial waves: further mathematical results, arXiv:1108.6194 [INSPIRE].

[11] A.B. Zamolodchikov, Conformal symmetry in two-dimensions: an explicit recurrence formula for the conformal partial wave amplitude, Commun. Math. Phys. 96 (1984) 419 [INSPIRE]. 
[12] F. Kos, D. Poland and D. Simmons-Duffin, Bootstrapping the $O(N)$ vector models, JHEP 06 (2014) 091 [arXiv: 1307.6856] [INSPIRE].

[13] J. Penedones, E. Trevisani and M. Yamazaki, Recursion relations for conformal blocks, JHEP 09 (2016) 070 [arXiv: 1509.00428] [INSPIRE].

[14] L. Iliesiu, F. Kos, D. Poland, S.S. Pufu, D. Simmons-Duffin and R. Yacoby, Fermion-scalar conformal blocks, JHEP 04 (2016) 074 [arXiv: 1511.01497] [INSPIRE].

[15] M.S. Costa, T. Hansen, J. Penedones and E. Trevisani, Radial expansion for spinning conformal blocks, JHEP 07 (2016) 057 [arXiv: 1603.05552] [INSPIRE].

[16] M.S. Costa, T. Hansen, J. Penedones and E. Trevisani, Projectors and seed conformal blocks for traceless mixed-symmetry tensors, JHEP 07 (2016) 018 [arXiv: 1603.05551] [INSPIRE].

[17] P. Kravchuk, Casimir recursion relations for general conformal blocks, JHEP 02 (2018) 011 [arXiv: 1709.05347] [INSPIRE].

[18] X. Zhou, Recursion relations in Witten diagrams and conformal partial waves, JHEP 05 (2019) 006 [arXiv: 1812.01006] [INSPIRE].

[19] R.S. Erramilli, L.V. Iliesiu and P. Kravchuk, Recursion relation for general 3d blocks, JHEP 12 (2019) 116 [arXiv:1907.11247] [INSPIRE].

[20] D. Simmons-Duffin, Projectors, shadows, and conformal blocks, JHEP 04 (2014) 146 [arXiv: 1204.3894] [INSPIRE].

[21] M.S. Costa, J. Penedones, D. Poland and S. Rychkov, Spinning conformal correlators, JHEP 11 (2011) 071 [arXiv: 1107.3554] [inSPIRE].

[22] M.S. Costa, J. Penedones, D. Poland and S. Rychkov, Spinning conformal blocks, JHEP 11 (2011) 154 [arXiv: 1109.6321] [INSPIRE].

[23] A. Castedo Echeverri, E. Elkhidir, D. Karateev and M. Serone, Deconstructing conformal blocks in $4 D$ CFT, JHEP 08 (2015) 101 [arXiv:1505.03750] [INSPIRE].

[24] A. Castedo Echeverri, E. Elkhidir, D. Karateev and M. Serone, Seed conformal blocks in $4 D$ CFT, JHEP 02 (2016) 183 [arXiv:1601.05325] [INSPIRE].

[25] D. Karateev, P. Kravchuk and D. Simmons-Duffin, Weight shifting operators and conformal blocks, JHEP 02 (2018) 081 [arXiv: 1706. 07813] [INSPIRE].

[26] G.F. Cuomo, D. Karateev and P. Kravchuk, General bootstrap equations in $4 D$ CFTs, JHEP 01 (2018) 130 [arXiv:1705.05401] [INSPIRE].

[27] H. Isono, On conformal correlators and blocks with spinors in general dimensions, Phys. Rev. D 96 (2017) 065011 [arXiv: 1706. 02835] [INSPIRE].

[28] J.-F. Fortin and W. Skiba, Conformal bootstrap in embedding space, Phys. Rev. D 93 (2016) 105047 [arXiv: 1602.05794] [INSPIRE].

[29] J.-F. Fortin and W. Skiba, A recipe for conformal blocks, arXiv:1905.00036 [INSPIRE].

[30] J.-F. Fortin and W. Skiba, New methods for conformal correlation functions, JHEP 06 (2020) 028 [arXiv: 1905.00434] [InSPIRE].

[31] J.-F. Fortin, V. Prilepina and W. Skiba, Conformal four-point correlation functions from the operator product expansion, JHEP 08 (2020) 115 [arXiv: 1907.10506] [INSPIRE].

[32] C. Sleight and M. Taronna, Spinning Witten diagrams, JHEP 06 (2017) 100 [arXiv:1702.08619] [INSPIRE]. 
[33] M.S. Costa and T. Hansen, AdS weight shifting operators, JHEP 09 (2018) 040 [arXiv: 1805.01492] [INSPIRE].

[34] M. Hogervorst, Dimensional reduction for conformal blocks, JHEP 09 (2016) 017 [arXiv: 1604.08913] [INSPIRE].

[35] A. Kaviraj, S. Rychkov and E. Trevisani, Random field Ising model and Parisi-Sourlas supersymmetry. Part I. Supersymmetric CFT, JHEP 04 (2020) 090 [arXiv:1912.01617] [INSPIRE].

[36] M. Besken, A. Hegde, E. Hijano and P. Kraus, Holographic conformal blocks from interacting Wilson lines, JHEP 08 (2016) 099 [arXiv: 1603.07317] [INSPIRE].

[37] A. Bhatta, P. Raman and N.V. Suryanarayana, Holographic conformal partial waves as gravitational open Wilson networks, JHEP 06 (2016) 119 [arXiv:1602.02962] [INSPIRE].

[38] A. Bhatta, P. Raman and N.V. Suryanarayana, Scalar blocks as gravitational Wilson networks, JHEP 12 (2018) 125 [arXiv:1806.05475] [INSPIRE].

[39] M. Isachenkov and V. Schomerus, Superintegrability of d-dimensional conformal blocks, Phys. Rev. Lett. 117 (2016) 071602 [arXiv: 1602.01858] [INSPIRE].

[40] V. Schomerus, E. Sobko and M. Isachenkov, Harmony of spinning conformal blocks, JHEP 03 (2017) 085 [arXiv: 1612.02479] [INSPIRE].

[41] I. Buric, V. Schomerus and E. Sobko, Superconformal blocks: general theory, JHEP 01 (2020) 159 [arXiv: 1904.04852] [inSPIRE].

[42] I. Burić, M. Isachenkov and V. Schomerus, Conformal group theory of tensor structures, JHEP 10 (2020) 004 [arXiv:1910.08099] [INSPIRE].

[43] E. Hijano, P. Kraus, E. Perlmutter and R. Snively, Witten diagrams revisited: the AdS geometry of conformal blocks, JHEP 01 (2016) 146 [arXiv:1508.00501] [INSPIRE].

[44] M. Nishida and K. Tamaoka, Geodesic Witten diagrams with an external spinning field, PTEP 2017 (2017) 053B06 [arXiv: 1609. 04563] [INSPIRE].

[45] A. Castro, E. Llabrés and F. Rejon-Barrera, Geodesic diagrams, gravitational interactions \& OPE structures, JHEP 06 (2017) 099 [arXiv: 1702.06128] [INSPIRE].

[46] E. Dyer, D.Z. Freedman and J. Sully, Spinning geodesic Witten diagrams, JHEP 11 (2017) 060 [arXiv: 1702.06139] [INSPIRE].

[47] H.-Y. Chen, E.-J. Kuo and H. Kyono, Anatomy of geodesic Witten diagrams, JHEP 05 (2017) 070 [arXiv: 1702.08818] [inSPIRE].

[48] S.S. Gubser and S. Parikh, Geodesic bulk diagrams on the Bruhat-Tits tree, Phys. Rev. D 96 (2017) 066024 [arXiv: 1704.01149] [INSPIRE].

[49] P. Kraus, A. Maloney, H. Maxfield, G.S. Ng and J.-Q. Wu, Witten diagrams for torus conformal blocks, JHEP 09 (2017) 149 [arXiv:1706.00047] [INSPIRE].

[50] K. Tamaoka, Geodesic Witten diagrams with antisymmetric tensor exchange, Phys. Rev. D 96 (2017) 086007 [arXiv: 1707.07934] [INSPIRE].

[51] M. Nishida and K. Tamaoka, Fermions in geodesic Witten diagrams, JHEP 07 (2018) 149 [arXiv: 1805.00217] [INSPIRE].

[52] S. Das, Comments on spinning OPE blocks in $A d S_{3} / C F T_{2}$, Phys. Lett. B 792 (2019) 397 [arXiv: 1811.09375] [INSPIRE]. 
[53] V. Rosenhaus, Multipoint conformal blocks in the comb channel, JHEP 02 (2019) 142 [arXiv: 1810.03244] [INSPIRE].

[54] D. Meltzer, E. Perlmutter and A. Sivaramakrishnan, Unitarity methods in AdS/CFT, JHEP 03 (2020) 061 [arXiv: 1912.09521] [INSPIRE].

[55] S. Parikh, Holographic dual of the five-point conformal block, JHEP 05 (2019) 051 [arXiv: 1901.01267] [INSPIRE].

[56] V. Gonçalves, R. Pereira and X. Zhou, $20^{\prime}$ five-point function from $A d S_{5} \times S^{5}$ supergravity, JHEP 10 (2019) 247 [arXiv:1906. 05305] [INSPIRE].

[57] C.B. Jepsen and S. Parikh, Propagator identities, holographic conformal blocks, and higher-point AdS diagrams, JHEP 10 (2019) 268 [arXiv:1906.08405] [INSPIRE].

[58] S. Parikh, A multipoint conformal block chain in d dimensions, JHEP 05 (2020) 120 [arXiv: 1911.09190] [INSPIRE].

[59] J.-F. Fortin, W.-J. Ma, V. Prilepina and W. Skiba, Efficient rules for all conformal blocks, arXiv:2002.09007 [INSPIRE].

[60] J.-F. Fortin, W. Ma and W. Skiba, Higher-point conformal blocks in the comb channel, JHEP 07 (2020) 213 [arXiv:1911.11046] [INSPIRE].

[61] J.-F. Fortin, W.-J. Ma and W. Skiba, Six-point conformal blocks in the snowflake channel, JHEP 11 (2020) 147 [arXiv: 2004. 02824] [INSPIRE].

[62] T. Anous and F.M. Haehl, On the Virasoro six-point identity block and chaos, JHEP 08 (2020) 002 [arXiv : 2005. 06440] [InSPIRE].

[63] A. Pal and K. Ray, Conformal correlation functions in four dimensions from quaternionic Lauricella system, arXiv:2005.12523 [INSPIRE].

[64] S. Albayrak and S. Kharel, Towards the higher point holographic momentum space amplitudes, JHEP 02 (2019) 040 [arXiv:1810.12459] [INSPIRE].

[65] S. Albayrak, C. Chowdhury and S. Kharel, New relation for Witten diagrams, JHEP 10 (2019) 274 [arXiv: 1904.10043] [INSPIRE].

[66] S. Albayrak and S. Kharel, Towards the higher point holographic momentum space amplitudes. Part II. Gravitons, JHEP 12 (2019) 135 [arXiv:1908.01835] [INSPIRE].

[67] S. Albayrak, C. Chowdhury and S. Kharel, Study of momentum space scalar amplitudes in AdS spacetime, Phys. Rev. D 101 (2020) 124043 [arXiv:2001.06777] [INSPIRE].

[68] A. Fitzpatrick, J. Kaplan, J. Penedones, S. Raju and B.C. van Rees, A natural language for AdS/CFT correlators, JHEP 11 (2011) 095 [arXiv:1107.1499] [INSPIRE].

[69] M.F. Paulos, Towards Feynman rules for Mellin amplitudes, JHEP 10 (2011) 074 [arXiv: 1107.1504] [INSPIRE].

[70] D. Nandan, A. Volovich and C. Wen, On Feynman rules for Mellin amplitudes in AdS/CFT, JHEP 05 (2012) 129 [arXiv: 1112.0305] [INSPIRE].

[71] G. Mack, D-dimensional conformal field theories with anomalous dimensions as dual resonance models, Bulg. J. Phys. 36 (2009) 214 [arXiv:0909.1024] [INSPIRE].

[72] G. Mack, D-independent representation of conformal field theories in D dimensions via transformation to auxiliary dual resonance models. Scalar amplitudes, arXiv:0907.2407 [INSPIRE]. 
[73] J. Penedones, Writing CFT correlation functions as AdS scattering amplitudes, JHEP 03 (2011) 025 [arXiv: 1011.1485] [INSPIRE].

[74] E.W. Barnes, A new development of the theory of the hypergeometric functions, Proc. Lond. Math. Soc. s2-6 (1908) 141.

[75] J.-F. Fortin, W.-J. Ma and W. Skiba, Seven-point conformal blocks in the extended snowflake channel and beyond, Phys. Rev. D 102 (2020) 125007 [arXiv:2006.13964] [InSPIRE].

[76] S. Kharel and G. Siopsis, Tree-level correlators of scalar and vector fields in AdS/CFT, JHEP 11 (2013) 159 [arXiv:1308.2515] [InSPIRE].

[77] V. Gonçalves, J. Penedones and E. Trevisani, Factorization of Mellin amplitudes, JHEP 10 (2015) 040 [arXiv: 1410.4185] [INSPIRE].

[78] J. Faller, S. Sarkar and M. Verma, Mellin amplitudes for fermionic conformal correlators, JHEP 03 (2018) 106 [arXiv:1711.07929] [INSPIRE].

[79] H.-Y. Chen, E.-J. Kuo and H. Kyono, Towards spinning Mellin amplitudes, Nucl. Phys. B 931 (2018) 291 [arXiv: 1712.07991] [INSPIRE].

[80] C. Sleight and M. Taronna, Spinning Mellin bootstrap: conformal partial waves, crossing kernels and applications, Fortsch. Phys. 66 (2018) 1800038 [arXiv:1804.09334] [INSPIRE].

[81] G. Lauricella, Sulle funzioni ipergeometriche a piu variabili (in Italian), Rend. Circ. Matem. Palermo 7 (1893) 111 [Erratum ibid. 7 (1893) 158].

[82] H.M. Srivastava and P.W. Karlsson, Multiple Gaussian hypergeometric series, Ellis Horwood, (1985).

[83] R.M. Aarts, Lauricella functions, from Math World - a Wolfram web resource, created by E.W. Weisstein, http://mathworld.wolfram.com/LauricellaFunctions.html.

[84] H. Exton, Multiple hypergeometric functions and applications, Ellis Horwood, (1976). 